University of Louisville

ThinkIR: The University of Louisville's Institutional Repository

8-2013

\title{
A numerical study of expressions for fill rate for single stage inventory system with periodic review.
}

Peiyu Luo

University of Louisville

Follow this and additional works at: https://ir.library.louisville.edu/etd

\section{Recommended Citation}

Luo, Peiyu, "A numerical study of expressions for fill rate for single stage inventory system with periodic review." (2013). Electronic Theses and Dissertations. Paper 866.

https://doi.org/10.18297/etd/866

This Master's Thesis is brought to you for free and open access by ThinkIR: The University of Louisville's Institutional Repository. It has been accepted for inclusion in Electronic Theses and Dissertations by an authorized administrator of ThinkIR: The University of Louisville's Institutional Repository. This title appears here courtesy of the author, who has retained all other copyrights. For more information, please contact thinkir@louisville.edu. 


\title{
A NUMERICAL STUDY OF EXPRESSIONS FOR FILL RATE FOR SINGLE STAGE
} INVENTORY SYSTEM WITH PERIODIC REVIEW

\author{
By
}

Peiyu Luo

B.E., Jiangnan University, 2008

\author{
A Thesis \\ Submitted to the Faculty of the \\ University of Louisville \\ J.B. Speed School of Engineering \\ as Partial Fulfillment of the Requirements \\ for the Professional Degree
}

Master of Science

Department of Industrial Engineering

University of Louisville

Louisville, Kentucky

August 2013 



\section{A NUMERICAL STUDY OF EXPRESSIONS FOR FILL RATE FOR SINGLE STAGE}

INVENTORY SYSTEM WITH PERIODIC REVIEW

Submitted by:

Peiyu Luo

A Thesis Approved on

$07 / 19 / 2013$

$\frac{07 / 19 / 2013}{(\text { Date })}$

by the following Reading and Examination Committee:

Dr. Lihui Bai, Thesis Director

Dr. Gerald W. Evans

Dr. Jian Guan 


\section{ACKNOWLEDGEMENTS}

I would like to express my deepest gratitude to my thesis advisor, Dr. Lihui Bai, for her never ending support and motivation. Her guidance along the way was really valuable and her patience and encouragements never failed to make me feel confident again in the research that I am doing. My sincere thanks go to Dr. Gerald Evans and Dr. Jian Guan for reviewing and providing some comments to improve this thesis. Dr. Jiang Zhang from the Robert B. Willumstad School of Business at Adelphi University has provided insightful discussions that I truly appreciate.

Special thanks to my dear wife, Yuan Zhang, for her unwavering love and motivation for me throughout my hard times in the journey to complete this thesis. My greatest appreciation goes to my beloved parents, Minxia Wang and Zhili Luo, for their love and support. Without their trust, I would have never reached this far.

Last but not least, I would like to thank my professors and fellow friends for their support, helpful comments and encouragement. Their help is deeply appreciated. 


\title{
ABSTRACT \\ A NUMERICAL STUDY OF EXPRESSIONS FOR FILL RATE FOR SINGLE STAGE INVENTORY SYSTEM WITH PERIODIC REVIEW
}

\author{
Peiyu Luo
}

July 19, 2013

Fill rate is one of the most important measurements for inventory systems in the supply chain management. The primary goal of this thesis is to give a comprehensive review of existing analytical expressions for the system fill rate, and provide numerical comparison for all relevant expressions in terms of their accuracy against (simulated) fill rate from the Monte Carlo simulation. We prove relationships between several expressions. Although majority of the expressions discussed herein are designed for standard periodic review system, we conduct numerical simulations for the general periodic review system. Under this general periodic review setting, numerical results indicate that all else being equal, replenishment lead time has larger effect on the system's fill rate than does the review interval. In addition, numerical comparison suggests that Johnson et al.'s approach, Zhang and Zhang's approach, Hadley and Whitin's approach dominate the traditional approach, exponential approximation and Silver's modified approach. The dominance is especially true for cases with high demand variability. For general periodic review system, our numerical results indicate that scaling 
is necessary for Silver's modified, Johnson et al.'s and Johnson et al.'s modified approaches. 


\section{TABLE OF CONTENTS}

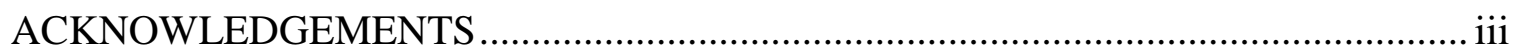

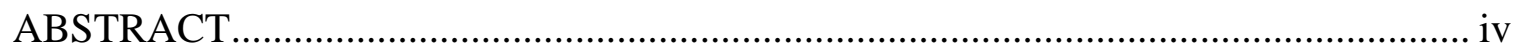

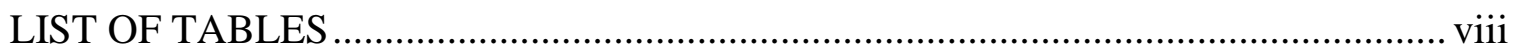

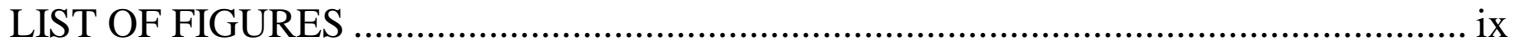

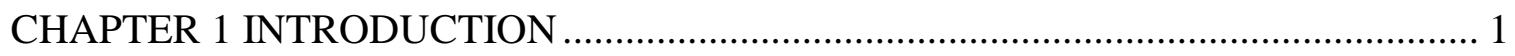

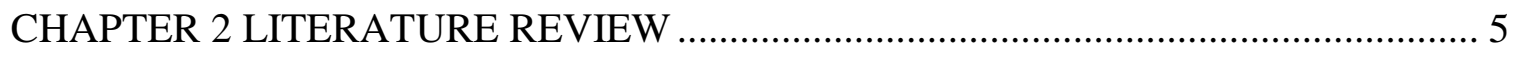

CHAPTER 3 EXPRESSIONS OF THE $\beta$ SERVICE LEVEL ......................................... 13

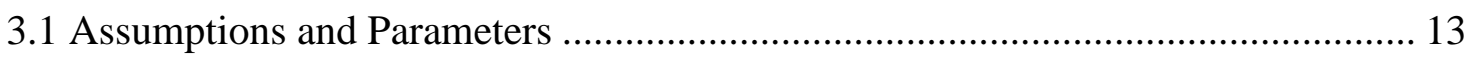

3.2 Traditional Approach and Its Exponential Approximation...................................... 14

3.3 Hadley and Whitin's Approach........................................................................ 17

3.3.1 An alternative approach by Silver and Bischak .............................................. 18

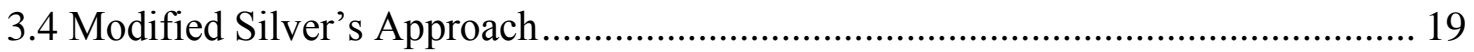

3.5 Johnson et al.'s approach ............................................................................ 22

3.5.1 Johnson et al.'s modified approach ........................................................... 24

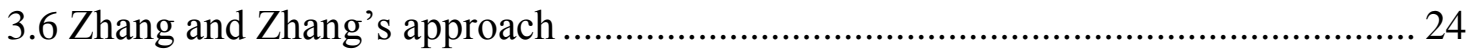

3.7 Scaling a General Periodic Review System .......................................................... 32

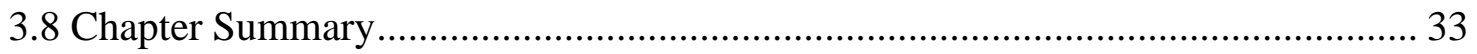


CHAPTER 4 DESIGN OF THE MONTE CARLO SIMULATION FOR SINGLESTAGE INVENTORY SYSTEMS WITH PERIODIC REVIEW AND BASE STOCK

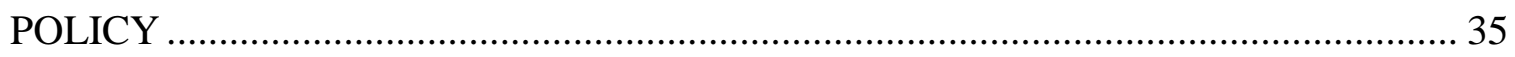

4.1 Inventory Dynamics and Chronology of Events ................................................ 35

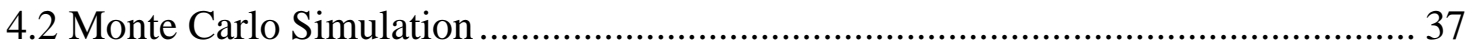

CHAPTER 5 NUMERICAL RESULTS ............................................................ 41

5.1 Results for the behavior of the system $\beta$ fill rate.......................................... 42

5.2 Results for evaluation of the $\beta$ service level expressions.................................... 47

5.2.1 Performance comparison with High Fill Rate ......................................... 47

5.2.2 Performance comparison with Low Fill Rate............................................ 52

5.2.3 Performance comparison when varying the $\mathrm{CV}$..................................... 55

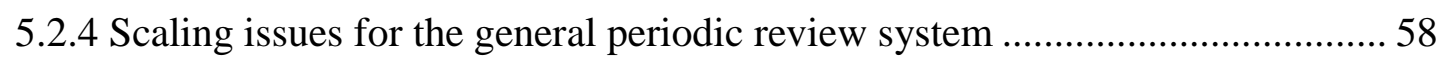

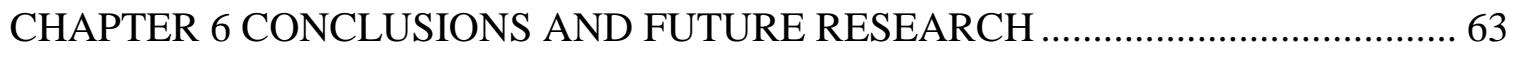

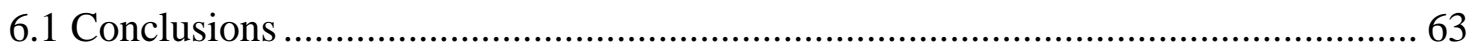

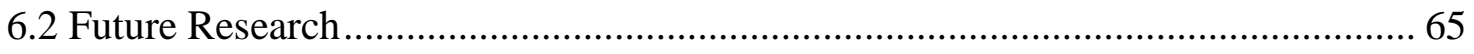

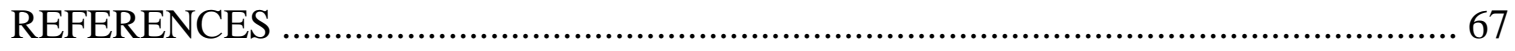

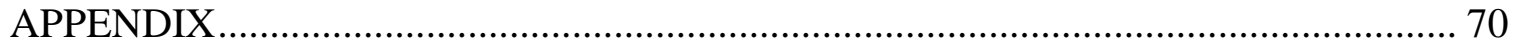

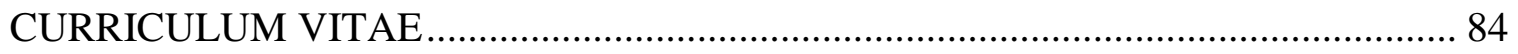




\section{LIST OF TABLES}

Table 3-1: An Illustrative Example of "Double Counting" ......................................... 16

Table 3-2: The Scaling Techniques for General Periodic Review System...................... 32

Table 3-3: Theoretical Comparison between All Formulas.............................................. 33

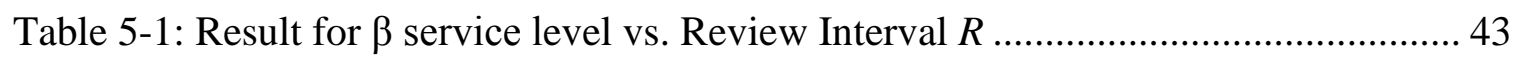

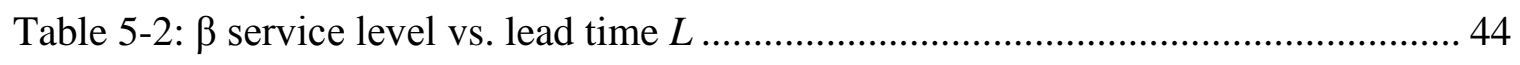

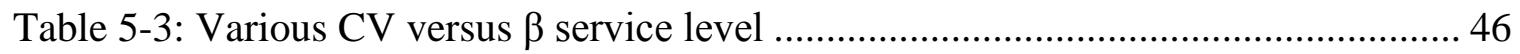

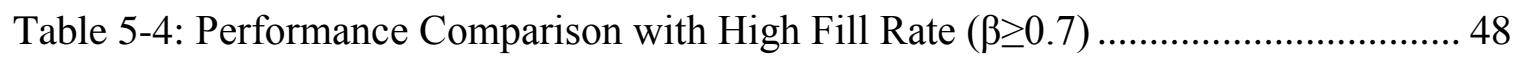

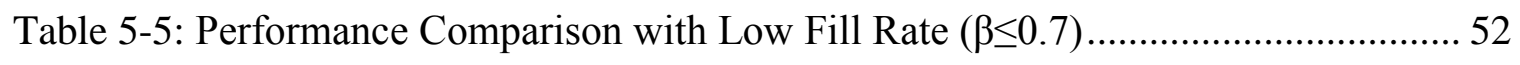

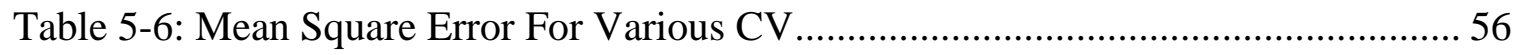

Table 5-7: Mean Square Error versus Monte Carlo simulation (truncated) .................... 58

Table 5-8: Unscaled parameter versus Scaled parameter .......................................... 59 


\section{LIST OF FIGURES}

Figure 5-1: Alpha Fill Rate versus Beta Fill Rate.................................................... 42

Figure 5-2: Various Lead Time versus Review Interval............................................. 45

Figure 5-3: Performance Comparison with High fill Rate........................................... 50

Figure 5-4: plot for Computation Results over MC results ......................................... 51

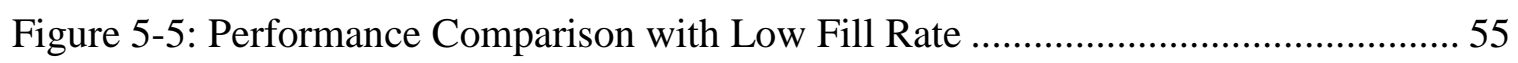

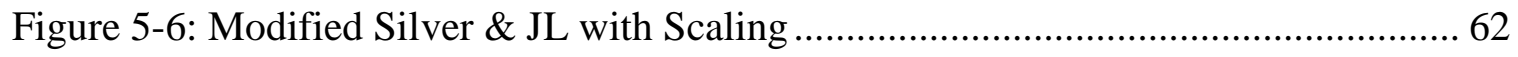

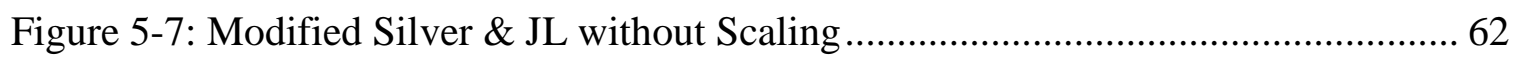




\section{CHAPTER 1 INTRODUCTION}

Fill rate, also known as service level, measures the performance of the inventory system in a supply chain. It is widely used by operations and/or inventory managers in real-world businesses (e.g., Tempelmeier 2000). Particularly, fill rate calculates the probability for a retailer or manufacturer to meet the customers' demands with on-hand inventory, or, equivalently not to face a stock out situation. For example, how many of the total demands can be satisfied without delay at a Best Buy's retail store? What is the probability for a Honda automobile dealer to meet various customers' demands during the whole sale year? It is inevitable that sometimes the inventory on hand is not sufficient to satisfy the customer demands immediately, especially under stochastic customer demands. As a result, these unfilled demands will be counted as "lost sales," which will not only result in penalty costs due to stock-out situation currently, but may also cause the retailer to lose the opportunity to sell to the same customers potentially in the future because of the reduced customer satisfaction. Thus, today's inventory management in supply chains requires the decision makers to focus on minimizing the total cost while achieving a desired service level in an inventory optimizing model.

In the literature (e.g., Tempelmeier 2000), service level can be categorized into three types. First, the $\alpha$-service level is an event-based measurement which describes the probability that all customers' demands can be satisfied immediately by the on hand inventory within a given period. In particular, two definitions of $\alpha$-service level are given 
with respect to different time intervals when customers' demands occur. Within a single demand period (e.g., one week, one month, etc.), $\alpha$-service level is the probability that the random customer demand during each single period is less or equal to the on hand inventory within the same period. On the other hand, if taking an ordering cycle as a demand period, $\alpha$-service level denotes the probability that the demand during replenishment lead time is less or equal to the on hand inventory at the beginning of lead time. In other words, in the latter definition, once a replenishment order is placed, the stock on hand must meet the demand during the lead time until previous orders arrival.

Second, the $\beta$ service level is a quantity-based measurement that not only describes the probability of a stock-out, but also provides an average expected number of backorders for every single demand period. Mathematically, $\beta$ service level is equal to the proportion of the total demand that is satisfied immediately by the on hand inventory. The $\beta$ service level is the most common service level measurement used in industry, thus it is the focus of the current thesis.

Finally, the $\gamma$-service level is defined as the proportion of total demand which is satisfied within each time period level. It not only calculates the amount of backorders but also the time until the backorders are filled. Compared to the above two service levels, this measurement is rarely used in industry.

In addition to the three different ways of calculating a firm's ability of meeting customers' demands immediately, the inventory literature also adopts two most common ways to track inventory for accounting and reordering purposes. They are the periodic review and continuous review inventory systems. Particularly, the periodic review system 
only tracks the inventory and makes ordering decision at every review interval. Such a system can help to reduce the reviewing cost while adding more uncertainty to the inventory, especially along with high-volume sale products. Accurate demand forecasting is required to prevent stock-out from occurring.

On the other hand, the continuous review system keeps tracking system inventory and updating inventory information all the time. A replenishment decision will be released when the inventory drops to a pre-determined level (i.e., reorder point). Continuous review system allows real-time updates for inventory counts, consequently it provides higher accuracy to the system inventory. However, the review of implementation cost is relatively higher than periodic review system.

Due to the important role service level plays in supply chain management and inventory management, researchers have worked extensively on developing formulas for calculating service level in a given inventory system. Such analytical formulas will facilitate relevant inventory optimizing models in quantitative supply chain management analysis. However, a comprehensive performance comparison among all formulas is lacking in the literature, which is greatly needed for inventory managers to make replenishment decisions. Thus, the current thesis aims to: 1) provide a state-of-the-art literature survey on analytical formulas for calculating the $\beta$ service level; 2) study the behavior of the $\beta$ service level for inventory systems under various settings (i.e., different base stock level, different lead times and review intervals, different coefficients of variation for stochastic demand); and 3) compare the accuracy of existing formulas under various settings. As a result, operations/inventory managers will be able to decide which 
formula(s) suits their inventory best, and ways (i.e., change of review interval; change of base stock level) to improve their customer service level.

In studying the true $\beta$ service level for a given inventory system, this thesis uses Monte Carlo simulation to create normally distributed random demand under the general periodic review using MATLAB (MATLAB 2002). In comparing existing formulas for fill rate, eight formulas in the literature are studied. They include tradition formula, exponential approximation, Hadley and Whitin's formula (1963), Silver's modified formula (1970), Johnson et al.'s formula and its modified form (1995), Zhang and Zhang's formula and its approximation (2006). All these formulas are also implemented in MATLAB (MATLAB 2002). The accuracy of these formulas is measured by the absolute value of the difference between the "true" (simulated) $\beta$ service level by the Monte Carlo simulation and the "calculated" $\beta$ service level by applying a formula. Furthermore, sensitivity analysis is performed to study the impact of lead time, review interval, coefficient of variation and base stock level on the accuracy of all relevant formulas. Finally, a unique discussion on how to properly scale an inventory system to best utilize various formulas is given in this thesis.

The rest of the thesis is organized as follows. Chapter 2 reviews the literature of inventory service level and supply chain optimization problems with service level constraints. Chapter 3 introduces the above-mentioned eight beta fill rate expressions from existing literatures; Chapter 4 presents the design of the Monte Carlo simulation. Chapter 5 discusses the numerical results of the Monte Carlo simulation and expressions presented in Chapters 3 and 4. Finally, Chapter 6 summarizes the thesis and discusses possible future work. 


\section{CHAPTER 2 LITERATURE REVIEW}

Service level, as an important performance measure in inventory management in supply chains, has been studied from several areas, including: availability monitoring in inventory control, service level constraints supply chain optimization and supply chain output performance measures (Beamon, 1999), to name a few. We will begin our literature review with these papers on the application of fill rate in inventory optimization models.

An earlier paper on service level effect on order levels in inventory model is published by Schneider in 1981. In practice, because of the uncertainty of stock-out cost, it is difficult to determine the optimal ordering policy. Consequently, the inventory analysis often faces two major problems: when to order and how much to order. Given a random demand variable which follows a particular distribution, by comparing three types of service level corresponding to fixed, proportional, time-independent stock out cost, Schneider successfully presented a method for determining an optimal or good inventory policy, using the appropriate measurements of effectiveness involving cost minimization.

Similarly, Silver (1995) investigated service and inventory implication with a heuristic rule which is commonly used by materials managers in North American distributor. That is, given the demands which are assumed to follow independent, 
identical distribution in $n$ periods, and the current value of reorder point is estimated by multiplying the maximum number of those observed demands with the replenishment lead time. Two service levels, $\alpha$ service level and $\beta$ service level are employed to evaluate the performance of manager's heuristic rule. They pointed out that both measurements can be converted to the functions of two parameters, i.e., the number of observed periods $\mathrm{n}$ and the length of replenishment lead time, $L$. More specifically, both alpha service level and beta service level will increase as the observation number $n$ increases. However, when the replenishment lead time $L$ increases, only alpha service level increases while beta service level will decrease, which means the probability for a stock out situation will be decreased but the expected backorders will increase. Alternatively, one can determine an appropriate number of observation periods $n$ for a desired service level with given lead time.

On the other hand, service level can be employed in a constraint in inventory optimization models. For example, Bashyam and Fu (1998) considered stochastic lead time for order crossing and service level constraint in an $(s, S)$ inventory system (where the inventory level is tracked periodically, once the inventory level falls below reorder point $s$, a new order will be placed to bring the inventory back to $S$.) with a penalty cost for unsatisfied demand. They developed a simulation based algorithm using the feasible directions approach from nonlinear programming. Particularly, the feasible direction approach in their work first applied an analytical approximation to initially set up the order quantity $Q$ by the well-known economic order quantity $(E O Q)$ model. Secondly, their approach did a line search on various reorder point $s$ while keeping the order quantity $Q$ constant by simulation. Finally, the feasible direction is updated using 
gradient estimation for the next iteration. Overall, their approach dominates current approaches to deal with managing $(s, S)$ inventory system that are subjected to high frequency of order crossing and can yield reasonable solutions within very short computation times.

Similarly, Ouyang and Wu (1996) proposed that lead time and order quantity can be modeled as decision variables in a "mixture" inventory system where backorders and lost sales are both allowed and taken into consideration in their model. Instead of a stockout term in the objective function, service level is a constraint of the minimization of the expected annual cost. Under their proposed algorithm, the expected annual cost is a function of order quantity $Q$ and replenishment lead time $L$. By setting the partial derivative of the expected annual cost function with respect to $Q$ to be zero, one obtains the optimal order quantity $Q^{*}$ for each value of the lead time $L^{*}$ subject to the service level constraint. Consequently, the optimal inventory policy can be found by comparing expected annual cost under different scenarios, i.e., combinations of ( $\left.Q^{*}, L^{*}\right)$.

Aside from fill rate application in inventory optimization models, other literatures concentrate on the development of exact fill rate formulas and how to use these formulas properly.

In reviewing existing formulas in the literature for computing the beta fill rate, perhaps the most simplistic formula is the so-called "traditional" formula for a periodic review inventory system under base stock policy (Nahmias, 1989). This "traditional" approach tracks the amount of demand that is not satisfied immediately by the on hand inventory in the system and its associated approximation is commonly used in operation 
management texts (e.g., see Chase et al, 1992; Vollman et al, 1988). Even though the traditional approach is easy to understand and apply, it will lead to two major concerns under some specific conditions. First, when the standard deviation of demand in lead time is high, this approach could produce negative fill rate value. This is because the standard deviation is one of the coefficients in calculating the expected shortage, and a sufficiently large value for the standard deviation can produce a negative fill rate.. Second, the traditional approach accounts the total shortage for every single period. Thus, if demand shortage occurs continuously in the system (which is very likely when the replenishment is not sufficient to fill the backorders), the estimated fill rate by this approach tends to overestimate the actual backorders for each period, thus underestimating the actual system fill rate.

Since the traditional approach, a more accurate approach has been developed by Hadley and Whitin (1963). The Hadley and Whitin's method uses Poisson distributed random demand and can be extended to normal distribution. In Hadley and Whitin's work, the expected shortage units for period is the average number of demands exceeds the base stock level within time $L+R$ (the lead time $L$ plus the review interval $R$ ) minus the average number of demands exceeds the base stock level within the lead time. This approach is accurate when the standard deviation of demand is small enough and when the review interval is smaller than the lead time.

In the literature, De Kok (1990) and Silver and Bischak (2011) use a similar approach as in Hadley and Whitin (1963). In particular, Silver and Bischak (2011) developed an exact fill rate formula which is essentially equivalent to Hadley \& Whitin's (1963). On the other hand, De Kok (1990) only proposed a similar approach without 
giving an exact fill rate formula. Furthermore, Silver and Bischak (2011) also provides an approximation method to determine the safety factor. Particularly, by omitting some terms in their formula, Silver and Bischak expressed the safety factor as a function of review interval, replenishment lead time and beta fill rate. Consequently, the approximation would overestimate the expected shortage units, thus the estimated safety factor will lead to a higher fill rate. Lastly, they indicate the relationship between expected shortage unit and safety factor could be used in a cost minimization model if the expected shortage units can be incorporated to the cost per unit short.

As experienced in the traditional as well as Silver and Bischak's approach, double counting re-occurring shortages is a lingering issue for an accurate computation of the fill rate. One potential solution is to divide the on hand inventory level for a periodic review system into three parts. Let $V$ denote to the amount of inventory that is available to satisfy further demands after any backorders are refilled, and $\mu$ denote the mean demand during lead time. Hence, there are three cases as follows:

1. If the demand $x$ during the replenishment lead time is less than base stock level $S$, then $V$ is equal to the demand during replenishment lead time $\mu * R$.

2. If the demand $x$ during the replenishment lead time exceeds the base stock level $S$ plus demand during replenishment lead time $\mu * R$, then $V$ is equal to 0 .

3. If the demand $x$ during the replenishment is between the base stock level $S$ and the base stock level $S$ plus the demand during replenishment lead time $\mu * R$, then $V$ is equal to $S+\mu * R-x$. 
With these three parts, one can calculate the expected shortage for each part individually. As a result, the maximum number of backorders is limited to be within $\mu$, thus the system fill rate avoids the double counting. However, this approach will overestimate the system fill rate because it limits the maximum shortage at $\mu$, whereas in practice the expected shortage will exceed the average demand per period.

Another solution to avoid double counting is offered by Johnson et al. (1985). In their paper, Johnson et al. who commented that the traditional expression for line item fill rate perform well for high rates (fill rates above 90\%) but consistently underestimate the true fill rate and will produce poor estimates as the fill rates decreases, again due to double counting previous backorders. Hence, based on their review and study of the expressions developed by Hadley and Whitin (1963), Johnson et al. (1985) developed an exact fill rate expression in periodic inventory systems, which can be expended to account for negative demand caused by high demand variability. The authors successfully showed that the modified version of their proposed formula dominates all other fill rate expressions when the demand variability is high.

Thus far, all the above-mentioned works deal with the standard (unit) periodic review system, in which the review interval is one unit of time. Although it can be argued that a general periodic review system $(R \neq 1)$ can be converted into a standard periodic review system by simple scaling, i.e., redefining the period demand to be the demand over $R$ periods, this scaling approach will not work when the lead time $(L)$ is not a multiple of review interval cycle $(R)$. In order to address this issue, Zhang and Zhang (2006) developed an exact fill rate of single-stage for general periodic review inventory systems, which introduce the general review inventory model and provides fill rate 
formulas for general demand distributions. In addition, Zhang and Zhang (2006) presented the specialized formulas when demand is normally distributed and two simplified approximations under normal demands. One approximation was based on basic properties of normal distribution and the other was based on a logistic distribution for the random demand.

Finally, we review several works on calculating fill rates for multi-stage inventory systems in supply chains. In today's competitive global supply chain environments, almost all end customers 'demands are met through a multistage system consisting of multiple tiers of suppliers, logistics brokers, wholesalers and retailers; such a multi-stage supply chain system could help to raise range from contractually distinct entities to multiple-stages of production in a single system. One effort to investigate the fill rate for multi-stage inventory systems is by Sobel (2004). He presented formulas to solve the fill rate problem of periodic review system on single-stage and multistage respectively.

In addition, Zhang et al. (2010) developed an exact fill rate expression of a general periodic review two-stage inventory system, which provides a much more general treatment for computing the fill rate computation method than other previous researchers. The numerical studies in Zhang et al. (2010) indicated that decreasing the lead time and increasing the echelon base stock level in the lower stage are more effective than adjusting these parameters in the upper stage level. The lead time and review period of lower stage plays a more critical role than higher stage as well when a higher system fill rate is desired. Finally Zhang et al. noted that their results could be extended to $N$ stage inventory system and a supply chain optimization problem where fill rate serves as a service-level constraint. 
Lastly, in a related work Thomas (2005) studied theoretical properties on the effect of review cycle in finite horizon on fill rate. Although most textbooks and software always assume the demand is stationary and serially independent for an infinite horizon, in practice, it is more likely to have a minimum fill rate realized within some finite review horizon, such as monthly or quarterly. His study suggested that the review cycle could affect a supplier's probability of achieving the target fill rate significantly over a fixed review horizon by implementing a finite horizon for measuring item fill rate performance. Furthermore, the utilization of finite-horizon, service-level contract could lead to a better performance of fill rate over the contractually specified target for a supplier.

In summary, this chapter reviews the existing literature on calculating the $\beta$ service level for single-stage as well as multi-stage inventory systems. Due to the limited works on the multi-stage inventory fill rate, this thesis focuses solely on simulating and evaluating the formulas for calculating the fill rate for single-stage inventory systems. In Chapter 3, we will discuss in detail the eight formulas for the $\beta$ service level under the normally distributed demand. Subsequently, in Chapter 4, we will design a Monte Carlo Simulation to simulate the inventory systems under various scenarios, aiming to evaluate the accuracy of all formulas reviewed in this chapter. 


\section{CHAPTER 3 EXPRESSIONS OF THE $\beta$ SERVICE LEVEL}

\subsection{Assumptions and Parameters}

This chapter focuses on presenting in details the eight main approaches to calculate the system fill rate for a periodic review system under the order up to policy. There are several common major assumptions made by all these approaches, and we list them as follows:

- The periodic demand is normally distributed with constant mean and standard deviation.

- Demands for different periods are identically and independently distributed.

- There is no lost sale and all demands exceeding on hand inventory are backlogged.

- The standard deviation for demand is sufficiently small to ensure no negative demand occurs.

- A replenishment order is placed once on hand inventory is tracked at every review interval.

- The replenishment lead time is constant.

The parameters in the following sections are defined as:

- $\mu$ denotes the mean value of the normally distributed demand 
- $\sigma$ denotes the standard deviation of the normally distributed demand

- $R$ denotes the length of the review interval in terms of numbers of time units (e.g., weeks, months, quarters)

- $L$ denotes the length of the replenishment lead time in terms of numbers of time units

- $\quad S$ denotes the order up to level (a.k.a., the base stock level)

- $f(x)$ denotes the probability density function for the standard normal distribution

- $F(x)$ denotes the cumulative density function for the standard normal distribution

- $G(x)$ denotes the unit normal loss function

- ESPC denotes the expected shortage per reviewing cycle

- $\beta$ denotes the system $\beta \beta$ service level

\subsection{Traditional Approach and Its Exponential Approximation}

The traditional approach is one of the most commonly used methods to estimate the system fill rate by measuring the units of demand that cannot be satisfied from the on hand inventory. The expected shortage per cycle (ESPC) is defined as:

$$
\begin{aligned}
& E S P C=\int_{S}^{\infty}\left(x_{0}-S\right) f_{x}\left(x_{0}\right) d x \\
& \text { Let } \mu_{0}=\frac{x_{0}-\mu(R+L)}{\sigma \sqrt{R+L}}, K(s)=\frac{S-\mu(R+L)}{\sigma \sqrt{R+L}} \\
& \text { Thus } d x_{0}=\sigma \sqrt{R+L} d \mu_{0}
\end{aligned}
$$


Substituting new variables into equation (1), one can obtain:

$$
\begin{aligned}
& E S P C=\int_{K(S)}^{\infty}(\sigma \sqrt{R+L}+\mu(R+L)-S) \frac{1}{\sqrt{2 \pi} \sigma \sqrt{R+L}} \exp \left(\frac{\left(x_{0}-\mu(R+L)\right)^{2}}{-2 \sigma^{2}(R+L)}\right) \sigma \sqrt{R+L} d \mu_{0} \\
& E S P C=\int_{K(S)}^{\infty} \sigma \sqrt{R+L}\left(\mu_{0}-\frac{S-\mu(R+L)}{\sigma \sqrt{R+L}}\right) \frac{1}{\sqrt{2 \pi}} \exp \left(\frac{\mu_{0}^{2}}{-2}\right) d \mu_{0} \\
& E S P C=\sigma \sqrt{R+L} \int_{K(S)}^{\infty}\left(\mu_{0}-\frac{S-\mu(R+L)}{\sigma \sqrt{R+L}}\right) \frac{1}{\sqrt{2 \pi}} \exp \left(\frac{\mu_{0}^{2}}{-2}\right) d \mu_{0}
\end{aligned}
$$

Applying the unit normal loss function $G(t)=\int_{t}^{\infty}(x-t) f(x) d x$ to equation (32), the system $\beta$ service level by the traditional approach, denoted as $\beta_{T}$, is given as:

$$
\beta_{T}=1-\frac{\sigma \sqrt{R+L} G(K(S))}{\mu}
$$

In order to simplify the calculation, several approximations haven been developed by eliminating the use of unit normal loss function $G(K(S))$. For example, Parr (1978) approximated equation (3-3) using the following:

$$
\beta_{E}=1-\frac{\sigma \sqrt{R+L} \exp \left(-0.92-1.19 K(S)-0.37 K^{2}(S)\right)}{\mu}
$$

Indeed, equation (3-4) essentially uses exponential functions to express the $\beta$ service level, thus is referred to as the "exponential approximation" in the literature. Thus, we denote it as $\beta_{E}$.

Note that "double counting" is the most significant drawback of the traditional approach. We illustrate the issue of double counting using an example from Templmeier (2000) in the following table. 
Table 3-1: An Illustrative Example of "Double Counting"

\begin{tabular}{|l|l|l|l|l|}
\hline Periods & Demand & Stock on hand & Backorders per & Unsatisfied \\
(after satisfying & period & demands \\
\hline 1 & 50 & 350 & - & 0 \\
\hline 2 & 58 & 292 & - & 0 \\
\hline 3 & 44 & 248 & - & 0 \\
\hline 4 & 59 & 189 & - & 0 \\
\hline 5 & 54 & 135 & - & 0 \\
\hline 6 & 50 & 85 & - & 0 \\
\hline 7 & 83 & 2 & -42 & 57 \\
\hline 9 & 44 & -99 & 99 & 0 \\
\hline
\end{tabular}

Note that in both periods 8 and 9, stock on hand is negative. Particularly, period 8 starts the backlog with 42 units, which will not be refilled after period 9. Thus, when demand in period 9 arrives, the total backlog for the system is 99 units and the actual backlog for period 9 is only 57 . However, the traditional approach would take all the demands that exceed base stock level (e.g., 42 and 99 in this example) into the integral. The latter implies that negative values of "stock on hand" will be accumulated via multiple periods 
and will be integrated for multiple times in the calculation. Thus, the "double counting" of back orders will overestimate the expected shortage per cycle.

\subsection{Hadley and Whitin's Approach}

As discussed in Chapter 2, several drawbacks of the traditional approach have inspired works for more accurate computation of the system fill rate. In 1963, Hadley and Whitin (1963) presented an approach to more accurately estimate the system fill rate by eliminating the double counting units. The ESPC in their approach is defined as: the total amount of backorders at the end of a particular cycle (review interval plus replenishment lead time) minus the total amount of backorders after replenishment arrival (lead time), and it can be expressed as:

$$
E S P C=\int_{S}^{\infty}\left(x_{0}-S\right) f_{x}\left(x_{0}\right) d x_{0}-\int_{S}^{\infty}\left(y_{0}-S\right) f_{y}\left(y_{0}\right) d y_{0}
$$

where $x_{0}$ is the demand during replenishment lead time plus review interval and $f_{x}(x)$ is the associated demand distribution density function, while $y_{0}$ is the demand during replenishment lead time and $f_{y}(y)$ is the associated demand density function. Similar to the traditional approach, Hadley and Whitin (1963) introduce new variables as:let $\mu_{0}=$ $\frac{x_{0}-\mu(R+L)}{\sigma \sqrt{R+L}}, K_{L+R}(s)=\frac{s-\mu(R+L)}{\sigma \sqrt{R+L}}$, thus one can have $d x_{0}=\sigma \sqrt{R+L} d \mu_{0}$; let $v_{0}=$ $\frac{y_{0}-\mu L}{\sigma \sqrt{L}}, K_{L}(s)=\frac{s-\mu L}{\sigma \sqrt{L}}$, then $d y_{0}=\sigma \sqrt{L} d v_{0}$.

Substituting these new variables into equation (3-5), one obtains:

$$
E S P C=\int_{K_{L+R}(S)}^{\infty}(\sigma \sqrt{R+L}+\mu(R+L)-S) \frac{1}{\sqrt{2 \pi} \sigma \sqrt{R+L}} \exp \left(\frac{\left(x_{0}-\mu(R+L)\right)^{2}}{-2 \sigma^{2}(R+L)}\right) \sigma \sqrt{R+L} d \mu_{0}
$$




$$
\begin{gathered}
-\int_{K_{L}(S)}^{\infty}(\sigma \sqrt{L}+\mu L-S) \frac{1}{\sqrt{2 \pi} \sigma \sqrt{R}} \exp \left(\frac{\left(y_{0}-\mu L\right)^{2}}{-2 \sigma^{2} L}\right) \sigma \sqrt{L} d v_{0} \\
E S P C=\int_{K_{L+R}(S)}^{\infty} \sigma \sqrt{R+L}\left(\mu_{0}-\frac{S-\mu(R+L)}{\sigma \sqrt{R+L}}\right) \frac{1}{\sqrt{2 \pi}} \exp \left(\frac{\mu_{0}^{2}}{-2}\right) d \mu_{0} \\
-\int_{K_{L}(S)}^{\infty} \sigma \sqrt{L}\left(\mu_{0}-\frac{S-\mu L}{\sigma \sqrt{L}}\right) \frac{1}{\sqrt{2 \pi}} \exp \left(\frac{v_{0}^{2}}{-2}\right) d v_{0} \\
E S P C=\sigma \sqrt{R+L} \int_{K_{L+R}(S)}^{\infty}\left(\mu_{0}-\frac{S-\mu(R+L)}{\sigma \sqrt{R+L}}\right) \frac{1}{\sqrt{2 \pi}} \exp \left(\frac{\mu_{0}^{2}}{-2}\right) d \mu_{0} \\
-\sigma \sqrt{L} \int_{K_{L}(S)}^{\infty}\left(\mu_{0}-\frac{S-\mu L}{\sigma \sqrt{L}}\right) \frac{1}{\sqrt{2 \pi}} \exp \left(\frac{v_{0}^{2}}{-2}\right) d v_{0}
\end{gathered}
$$

Using the unit normal loss function $G(t)=\int_{t}^{\infty}(x-t) f(x) d x$, the $\beta$ service level is given as:

$$
\beta_{H}=1-\frac{\sigma \sqrt{R+L} G\left(K_{L+R}(S)\right)-\sigma \sqrt{L} G\left(K_{L}(S)\right)}{\mu}
$$

\subsubsection{An alternative approach by Silver and Bischak}

More recently, Silver and Bischak (2011) proposed another formula which essentially is equivalent to Hadley and Whitin's formula. In Silver and Bischak (2011), the $\beta$ service level is defined as:

$$
\beta_{S B}=1-\frac{E S U}{\mu R}
$$

where ESU represents the expected shortage units and is calculated as $E S U=$ $\sqrt{L+R} \sigma G(k)-\sqrt{L} \sigma G\left(\frac{R}{\sqrt{L} C V}++k \sqrt{\frac{R+L}{L}}\right) \quad$ with $\quad C V=\frac{\sigma}{\mu}$, the safety factor being $k=$ 
$\frac{S-(L+R) \mu}{\sqrt{L+R} \sigma}$, and $G(k)$ being the unit normal loss function. When substituting $E S U, k$, and $C V$ into equation (3-7), one obtains:

$$
\begin{gathered}
\beta_{S B}=1-\left\{\frac{\sqrt{L+R} \sigma G\left(\frac{S-(L+R) \mu}{\sqrt{L+R} \sigma}\right)-\sqrt{L} \sigma G\left(\frac{R \mu}{\sqrt{L} \sigma}+\frac{S-(L+R) \mu}{\sqrt{L+R} \sigma} \sqrt{\frac{R+L}{L}}\right)}{\mu R}\right\} \\
\beta_{S B}=1-\left\{\frac{\sqrt{L+R} \sigma G\left(\frac{S-(L+R) \mu}{\sqrt{L+R} \sigma}\right)-\sqrt{L} \sigma G\left(\frac{S-L \mu}{\sqrt{L} \sigma}\right)}{\mu R}\right\}
\end{gathered}
$$

Finally, it is worth mentioning the scaling issue associated with Silver and Bischak (2011). Note that in (3-8), parameter $R$ is the review interval and can be eliminated, i.e., scaled to a unit length 1 , by redefining the inventory system's unit time to be $R$. If one redefines $R$ as the unit time, then equation (3-8) becomes:

$$
\beta_{S B}=1-\left\{\frac{\sqrt{L+R} \sigma G\left(\frac{S-(L+R) \mu}{\sqrt{L+R} \sigma}\right)-\sqrt{L} \sigma G\left(\frac{S-L \mu}{\sqrt{L} \sigma}\right)}{\mu}\right\}
$$

Theorem 3.1. Silver and Bischak (2011)'s expression is equivalent to Hadley and Whitin (1963)'s.

Proof. Compare equations (3-9) with (3-6), the result follows immediately.

\subsection{Modified Silver's Approach}

Like Hadley and Whitin (1963), Silver (1970) also attempted to eliminate the problem of double counting previous backorders for a continuously reviewed inventory system. Silver (1970)'s approach is to examine the inventory immediately after a replenishment order (of size $Q$ ) arrives. In particular, let $V$ denote the item units 
available after any previous backorders are satisfied and $x$ denote the demand during the replenishment lead time, thus the amount of available units $V$ can be expressed as:

$$
V(Q)=\left\{\begin{aligned}
Q, & s \geq x \\
s+Q-x, & s+Q>x>s \\
0, & x \geq s+Q
\end{aligned}\right.
$$

Accordingly, the expected value of units that can be used to satisfy demand is:

$E(V(Q))=\int_{-\infty}^{s} Q f(x) d x+\int_{s}^{s+Q}(s+Q-x) f(x) d x+\int_{s+Q}^{\infty} 0 f(x) d x$

Consequently, $\beta$ service level for the continuously reviewed system is:

$$
\beta=\frac{E(V)}{Q}
$$

Note that equation (3-10) is the expected value of units that can be used to satisfy demand after any backorders are refilled. When discussing a periodic review system, Johnson et al. (1995) made the following transformations and the resulting formula is termed as the "modified Silver's approach" in their paper.

- The reorder point $s$ in a continuous review system is equal to the base stock level S in the corresponding periodic review system. Since both of reorder point and base stock level are the maximum inventory level when a particular cycle begins with.

- The replenishment lead time in a continuous review system is equal to review interval plus replenishment lead time in the corresponding periodic review system $(R+L)$. Thus $R+L$ becomes "effective" replenishment lead 
time and the inventory level is tracked all the time in the periodic review system.

- The replenishment order quantity $Q$ in a continuous review system is equal to demands occur during the review interval in the corresponding periodic review system $(\mu R)$. This is because we have converted the review interval plus the replenishment lead time to become the "effective" lead time.

With the above transformations between continuous and periodic review systems, the expected value of units that can be used to satisfy demand after any backorders refilled in a periodic review system can be expressed as:

$$
E(V(S))=\int_{-\infty}^{S} \mu R f(x) d x+\int_{S}^{S+\mu R}(s+\mu R-x) f(x) d x+\int_{S+\mu R}^{\infty} 0 f(x) d x
$$

Note that in (3-12) $x$ is the demand during the "effective" lead time. Therefore, the “modified Silver's" $\beta$ service level for the periodic review system is given as:

$$
\beta_{S}==\frac{E(V(S))}{\mu R}
$$

However, Johnson et al. (1995) note that equation (3-13) is not the accurate fill rate for a periodic review system due to the assumption that one orders exact $Q$ replenishment units for each review interval. In fact, for a practical periodic review system, the order quantity can vary due to anticipated change of stochastic demand. Furthermore, Johnson et al. (1995) illustrated the potential problem with a special case when $R=1$. When $R=1$, 


$$
\begin{gathered}
\beta_{S}=\frac{E(V)}{\mu} \\
1-\beta=1-\frac{E(V)}{\mu} \\
\text { and } \mu(1-\beta)=\mu\left(1-\frac{E(V)}{\mu}\right)=\mu-E(V) .
\end{gathered}
$$

Note that $\mu(1-\beta)$ represents the expected shortage per cycle, thus the following holds:

$$
\begin{gathered}
E S P C=\int_{-\infty}^{S}(\mu-\mu) f(x) d x+\int_{S}^{S+\mu}(\mu-s-\mu+x) f(x) d x+\int_{S+\mu}^{\infty}(\mu-0) f(x) d x \\
=\int_{S}^{S+\mu}(x-S) f(x) d x+\int_{S+\mu}^{\infty} \boldsymbol{\mu} f(x) d x
\end{gathered}
$$

As noted by Johnson et al. (1995), the above special case shows that Silver's modified method essentially truncates the maximum number of back orders at $\mu$, thus underestimating the true expected shortage and overestimating the true system fill rate.

\subsection{Johnson et al.'s approach}

Upon acknowledging the effort of eliminating the problem of double counting by Silver (1970) and discovering the potential inaccuracy issue with Silver's approach, Johnson et al. (1995), derived an exact fill rate expression by calculating the expected shortage units for one period of demand which is not fulfilled. In particular, at a given time period $t$, let $w$ be the demand in one period and $z$ be the total demand in $R+L-1$ periods. Hence there are two cases: 
1. If the demands at time period $t+R+L-1$ are more than the base stock level $S$, then stock out will occur for period $t+R+L$, and the demand for this period will be backordered.

2. If the inventory at period $t+R+L-1$ is positive, then the backorder units is the expected value of total demand $w+z$ that exceeds base stock level $S$ within time period $t+R+L$.

With the above considerations, Johnson and coauthors gave their ESPC expression as:

$$
E S P C=\int_{z_{0}=-\infty}^{S} \int_{w_{0}=S-z_{0}}^{\infty}\left(w_{0}+z_{0}-S\right) f_{z}\left(z_{0}\right) f_{w}\left(w_{0}\right) d w_{0} d z_{0}+\mu P_{\geq}\left(\frac{S-(R+L-1) \mu}{\sigma \sqrt{R+L-1}}\right),
$$

where $\quad P_{\geq}\left(\frac{S-(R+L-1) \mu}{\sigma \sqrt{R+L-1}}\right)=1-f\left(\frac{S-(R+L-1) \mu}{\sigma \sqrt{R+L-1}}\right) . \quad$ In $\quad$ the above equation, Let $t=$ $\frac{w_{0}-\mu}{\sigma}$ and $y=\frac{z-\mu(R+L-1)}{\sigma \sqrt{R+L-1}}$, then $d w_{0}=\sigma d t$ and $d z_{0}=\sigma \sqrt{R+L-1} d y$

Additionally, letting $g=\sigma \sqrt{R+L-1}$, the original double integral can be re-expressed as:

$$
\frac{1}{2 \pi} \int_{y=-\infty}^{\frac{S-(R+L-1) \mu}{g}} \int_{t=\frac{S-y g-(R+L-1) \mu}{\sigma}}^{\infty}\left(t-\frac{S-y g-(R+L-1) \mu}{\sigma}\right) \exp \left(-\frac{y^{2}+t^{2}}{2}\right) d t d y
$$

Using the unit normal loss function $G(t)=\int_{t}^{\infty}(x-t) f(x) d x$ and adding the probability term, one then has:

$$
E S P C=\frac{\sigma}{2 \pi} \int_{y=-\infty}^{\frac{S-(R+L-1) \mu}{g}} G\left(\frac{S-y g-(R+L-1) \mu}{\sigma}\right) \exp \left(-\frac{y^{2}}{2}\right) d y+\mu P_{\geq}\left(\frac{S-(R+L-1) \mu}{\sigma \sqrt{R+L-1}}\right)
$$

Thus, system fill rate is given as: 


$$
\beta_{J}=1-\frac{E S P C}{\mu}
$$

\subsubsection{Johnson et al.'s modified approach}

Johnson et al (1995) noted that their approach produces the same estimates as Hadley and Whitin's approach for cases of low demand variability. Thus, their modified approach attempts to address the case of high demand variability. When the demand variability is high, i.e., the standard deviation of demand is large relative to its mean, the probability of incurring negative demand is also high. One practical example used by Johnson et al. (1995) for this scenario is the case from Hewlett-Packard, where it is not unusual to observe high volume of customer returns that makes the period demand negative. In order to address high demand variability, Johnson et al. slightly modified their exact fill rate expression as:

$$
\begin{aligned}
E S P C_{\text {modified }} & =\int_{z_{0}=-\infty}^{S} \int_{w_{0}=S-z_{0}}^{\infty}\left(w_{0}+z_{0}-S\right) f_{z}\left(z_{0}\right) f_{w}\left(w_{0}\right) d w_{0} d z_{0} \\
& +\int_{w_{o}=0}^{\infty} w_{0} f_{w}\left(w_{0}\right) P_{\geq}\left(\frac{S-(R+L-1) \mu}{\sigma \sqrt{R+L-1}}\right)
\end{aligned}
$$

Consequently, the modified formula for the system $\beta$ service level becomes:

$$
\beta_{J M}=1-\frac{E S P C_{\text {modified }}}{\int_{w_{o}=0}^{\infty} w_{0} f_{w}\left(w_{0}\right)}
$$

\subsection{Zhang and Zhang's approach}

Thus far, all formulas introduced only deal with the standard (or unit) review interval for the periodic review inventory system. It has been argued that a general 
periodic review system (where the review interval $R \geq 1$ ) can be converted to the standard (unit) periodic review system (where review interval $R=1$ ) by the simple scaling technique. However, this scaling technique only works when the replenishment lead time $L$ is a multiple of the review interval $R$. For instance, consider a periodic review system with the replenishment lead time $L=4$ days, the review interval $R=2$ days, and the normal demand with mean $\mu=200$ per day and standard deviation $\sigma=100$ per day. If we scale the time for this inventory system by redefining the unit time to be 2 days instead of 1 day, then the effective review interval becomes $R^{\prime}=1$ and replenishment lead time $L^{\prime}=2$, and the effective period demand mean $\mu^{\prime}=200 * 2=400$ and standard deviation $\sigma^{\prime}=100 * \sqrt{2}=141.4$. However, if the replenishment lead time is not a multiple of the review interval (e.g., $L=5$ and $R=2$ ), this simple scaling approach is hard to implement. In fact, our simulation results in Chapter 5 suggest that using effective lead time $L^{\prime}=2.5$, or $L^{\prime}=2$ (rounding down), or $L^{\prime}=3$ (rounding up) will likely lead to inaccurate fill rate.

In order to address the general review for an inventory system, Zhang and Zhang (2006) presented an exact fill rate formula for the so called "general periodic review system". Unlike above mentioned exact fill rate formulas, they estimate $\beta$ service level by counting the long run average fraction of total demand that can be satisfied from on hand inventory rather than the amount of expected shortage units, which is given as:

$$
\beta_{Z Z}=\frac{1}{R \mu} \int_{0}^{S}\left(\Phi^{(L)}\left(b_{L}\right)-\Phi^{(L+R)}\left(b_{L+R}\right)\right) d b
$$

where $\Phi^{(L)}(b), \Phi^{(L+R)}(b)$ are the $L$-fold and $L+R$-fold convolution of the cumulative density function of normal distribution; $b_{L}, b_{L+R}$ represent the demand during replenishment lead time and replenishment lead time plus review interval, respectively. 
In order to keep the consistency in this thesis, we use $F(x)$ and $f(x)$ to replace the cumulative density function and probability density function of demands $\Phi(x)$ and $\phi(x)$, respectively. Because $b_{L}=\frac{x-L \mu}{\sigma \sqrt{L}}$ and $b_{L+R}=\frac{x-(L+R) \mu}{\sigma \sqrt{L+R}} \quad, d b_{L}=\sigma \sqrt{L} d x$ and $d b_{L+R}=$ $\sigma \sqrt{L+R} d x$. Thus, (3-18) reduces to:

$\beta_{Z Z}=\frac{1}{R \mu}\left(\sigma \sqrt{L} \int_{\frac{-L \mu}{\sigma \sqrt{L}}}^{\frac{S-L \mu}{\sigma \sqrt{L}}} F(x) d x-\sigma \sqrt{L+R} \int_{\frac{-(L+R) \mu}{\sigma \sqrt{L+R}}}^{\frac{S-(L+R) \mu}{\sigma \sqrt{L+R}}} F(x) d x\right)$

According to Hadley and Whitin (1963), $\int_{t}^{\infty}\{1-F(x)\} d x=f(t)+t F(t)-t$, thus one obtains the following:

$$
\begin{gathered}
\int_{t}^{\infty}\{1-F(x)\} d x=\int_{t}^{\infty} 1 d x-\int_{t}^{\infty} F(x) d x \\
\int_{t}^{\infty} 1 d x-\int_{t}^{\infty} F(x) d x=f(t)+t F(t)-t \\
\int_{t}^{s} 1 d x+\int_{s}^{\infty} 1 d x-\int_{t}^{s} F(x) d x-\int_{s}^{\infty} F(x) d x=f(t)+t F(t)-t \\
(S-t)-\int_{s}^{\infty}\{1-F(x)\} d x-\int_{t}^{s} F(x) d x=f(t)+t F(t)-t \\
(S-t)+f(S)+S F(S)-S-\int_{t}^{s} F(x) d x=f(t)+t F(t)-t \\
\int_{t}^{s} F(x) d x=f(s)-f(t)+S F(s)-t F(t)
\end{gathered}
$$

It follows from simple algebra after substituting (3-20) into equation (3-19) that: 


$$
\begin{aligned}
& \beta_{Z Z}=\frac{1}{R \mu}\left\{\sigma \sqrt{L}\left[f\left(\frac{S-L \mu}{\sigma \sqrt{L}}\right)-f\left(\frac{-L \mu}{\sigma \sqrt{L}}\right)\right]-\sigma \sqrt{L+R}\left[f\left(\frac{S-(L+R) \mu}{\sigma \sqrt{L+R}}\right)-f\left(\frac{-(L+R) \mu}{\sigma \sqrt{L+R}}\right)\right]+\right. \\
& (S-L \mu)\left[F\left(\frac{S-L \mu}{\sigma \sqrt{L}}\right)-F\left(\frac{S-(L+R) \mu}{\sigma \sqrt{L+R}}\right)\right]+R \mu F\left(\frac{S-(L+R) \mu}{\sigma \sqrt{L+R}}\right)+L \mu F\left(\frac{-L \mu}{\sigma \sqrt{L}}\right)-(L+ \\
& \left.R) \mu F\left(\frac{-(L+R) \mu}{\sigma \sqrt{L+R}}\right)\right\}
\end{aligned}
$$

Note that equation (3-21) only involves the probability density function and cumulative density function of the normal distribution, thus it is easy to implement in a spread sheet in practice. Apart from the exact fill rate expression, Zhang et al. (2006) also presented an approximation based on the properties of the normal distribution. Particularly, the following approximations are appropriate given the normally distributed demand. Additionally,

$$
f\left(\frac{-L \mu}{\sigma \sqrt{L}}\right) \cong 0, f\left(\frac{-(L+R) \mu}{\sigma \sqrt{L+R}}\right) \cong 0, F\left(\frac{-L \mu}{\sigma \sqrt{L}}\right) \cong 0, F\left(\frac{-(L+R) \mu}{\sigma \sqrt{L+R}}\right) \cong 0
$$

in a periodic review system, the base stock level usually satisfies $S \gg L \mu$, which implies: $f\left(\frac{S-L \mu}{\sigma \sqrt{L}}\right) \cong 0$ and $F\left(\frac{S-L \mu}{\sigma \sqrt{L}}\right) \cong 1$.

With the above simplifications, the approximated fill rate expression can be rewritten as:

$$
\begin{aligned}
& \beta_{Z Z A p p}= \\
& \frac{1}{R \mu}\left\{-\sigma \sqrt{L+R} f\left(\frac{S-(L+R) \mu}{\sigma \sqrt{L+R}}\right)+(S-L \mu)\left[1-F\left(\frac{S-(L+R) \mu}{\sigma \sqrt{L+R}}\right)\right]+R \mu F\left(\frac{S-(L+R) \mu}{\sigma \sqrt{L+R}}\right)\right\}
\end{aligned}
$$

Recalling $K(s)=\frac{S-\mu(R+L)}{\sigma \sqrt{R+L}}$ and the unit normal loss function, one then obtains:

$$
\beta_{Z \& Z A p p}=1-\frac{1}{R \mu} \sigma \sqrt{L+R}[f(K(s))-K(s)(1-F(K(s))]
$$




$$
=1-\frac{1}{R \mu} \sigma \sqrt{L+R} G(K(s))
$$

Finally note that when $R$ is to be eliminated by redefining $R$ to be the time unit of the inventory system, Zhang and Zhang's approximation (3-22) is equivalent to the traditional approach (3-3), which is supported by our numerical results in Chapter 5.

Theorem 3.2. The traditional approach (3-3) is equivalent to Zhang and Zhang's approximation, i.e., equation (9) in Zhang and Zhang (2006).

Proof. Let $z=\frac{S-(L+R) \mu}{\sqrt{L+R} \sigma}=K(S)$ and $\tau=S$, then the approximation expression (9) in Zhang and Zhang (2006) is as follows:

$$
\begin{aligned}
\beta_{Z Z A p p}= & \frac{1}{R \mu}\{-\sigma \sqrt{L+R} f(K(S))+(S-L \mu)-(S-L \mu) F(K(S))+R \mu F(K(S))\} \\
& =\frac{1}{R \mu}\{-\sigma \sqrt{L+R} f(K(S))+(S-L \mu)-(S-(L+R) \mu) F(K(S))\} \\
& =\frac{-\sigma \sqrt{L+R}}{R \mu}\left\{f(K(S))-\frac{(S-L \mu)}{\sigma \sqrt{L+R}}+\frac{S-(L+R) \mu}{\sqrt{L+R} \sigma} F(K(S))\right\} \\
& =1-\frac{\sigma \sqrt{L+R}}{R \mu}\left\{f(K(S))+\frac{R \mu}{\sigma \sqrt{L+R}}-\frac{(S-L \mu)}{\sigma \sqrt{L+R}}+K(S) F(K(S))\right\} \\
& =1-\frac{\sigma \sqrt{L+R}}{R \mu}\left\{f(K(S))-\frac{S-L \mu-R \mu}{\sigma \sqrt{L+R}}+K(S) F(K(S))\right\} \\
& =1-\frac{\sigma \sqrt{L+R}}{R \mu}\{f(K(S))-K(S)[1-F(K(S))]\} \\
& =1-\frac{\sigma \sqrt{L+R}}{R \mu} G(K(S))
\end{aligned}
$$


On the other hand, for a general periodic review with $R \neq 1$, one can redefine the unit time interval to be $R$ periods instead of 1 . After this scaling, directly applying $\beta_{T}$ as in equation (3-3) becomes:

$\beta_{T}=1-\frac{\sigma \sqrt{R+L} G(K(S))}{\mu R}$

Then it follows that (3-23) is equivalent to (3-24), thus the equivalency between Zhang and Zhang's approximation and the traditional approach for a general periodic review inventory system.

Next we discuss the relationship between Zhang and Zhang's approach, its approximation and Silver and Bischak (2011)'s results.

Lemma 3. 1. If $-\sigma \sqrt{L} f\left(\frac{-L \mu}{\sigma \sqrt{L}}\right)+\sigma \sqrt{L+R} f\left(\frac{-(L+R) \mu}{\sigma \sqrt{L+R}}\right)+L \mu F\left(\frac{-L \mu}{\sigma \sqrt{L}}\right)-(L+$ $R) \mu F\left(\frac{-(L+R) \mu}{\sigma \sqrt{L+R}}\right) \geq 0$, then $\beta_{Z Z} \geq \beta_{S B}$.

Proof. Recall Equation (3-7), the exact $\beta$ fill rate expression in Silver and Bischak (2011) can be expressed as:

$$
\beta_{S B}=1-\left\{\frac{\sqrt{L+R} \sigma G\left(\frac{S-(L+R) \mu}{\sqrt{L+R} \sigma}\right)-\sqrt{L} \sigma G\left(\frac{R \mu}{\sqrt{L} \sigma}+\frac{S-(L+R) \mu}{\sqrt{L+R} \sigma} \sqrt{\frac{R+L}{L}}\right)}{\mu R}\right\}
$$

Applying the unit normal loss function $G(t)=f(t)-t[1-F(t)]$, one obtains:

$$
\begin{aligned}
& \beta_{S B}=1-\frac{\sigma \sqrt{L+R}}{R \mu}\left\{f\left(\frac{S-(L+R) \mu}{\sqrt{L+R} \sigma}\right)-\frac{S-(L+R) \mu}{\sqrt{L+R} \sigma}+\frac{S-(L+R) \mu}{\sqrt{L+R} \sigma} F\left(\frac{S-(L+R) \mu}{\sqrt{L+R} \sigma}\right)\right\}+\frac{\sigma \sqrt{L}}{R \mu}\left\{f\left(\frac{S-L \mu}{\sqrt{L+R} \sigma}\right)-\right. \\
& \left.\frac{S-L \mu}{\sqrt{L} \sigma}+\frac{S-L \mu}{\sqrt{L} \sigma} F\left(\frac{S-L \mu}{\sqrt{L} \sigma}\right)\right\} .
\end{aligned}
$$


Thus, $\beta_{S B}=\frac{1}{R \mu}\left\{\sigma \sqrt{L} f\left(\frac{S-L \mu}{\sqrt{L+R} \mu}\right)-\sigma \sqrt{L} f\left(\frac{S-(L+R) \mu}{\sqrt{L+R} \mu}\right)-S F\left(\frac{S-(L+R) \mu}{\sqrt{L+R} \mu}\right)+(L+\right.$ R) $\left.\mu F\left(\frac{S-(L+R) \mu}{\sqrt{L+R} \mu}\right)+S F\left(\frac{S-L \mu}{\sigma \sqrt{L}}\right)-L \mu F\left(\frac{S-L \mu}{\sigma \sqrt{L}}\right)\right\}$.

Then it follows: $\beta_{S B}=\frac{1}{R \mu}\left\{-\sigma \sqrt{L+R} f\left(\frac{S-(L+R) \mu}{\sigma \sqrt{L+R}}\right)-(S-L \mu) F\left(\frac{S-(L+R) \mu}{\sigma \sqrt{L+R}}\right)+\right.$

$\left.R \mu F\left(\frac{S-(L+R) \mu}{\sigma \sqrt{L+R}}\right)\right\}+\frac{1}{R \mu}\left\{\sigma \sqrt{L} f\left(\frac{S-L \mu}{\sigma \sqrt{L}}\right)+(S-L \mu) F\left(\frac{S-L \mu}{\sigma \sqrt{L}}\right)\right\}$

Recall Zhang and Zhang's exact fill rate expression in Equation (3-21)

$$
\begin{aligned}
& \beta_{Z Z}=\frac{1}{R \mu}\left\{\sigma \sqrt{L}\left[f\left(\frac{S-L \mu}{\sigma \sqrt{L}}\right)-f\left(\frac{-L \mu}{\sigma \sqrt{L}}\right)\right]-\sigma \sqrt{L+R}\left[f\left(\frac{S-(L+R) \mu}{\sigma \sqrt{L+R}}\right)-f\left(\frac{-(L+R) \mu}{\sigma \sqrt{L+R}}\right)\right]+\right. \\
& (S-L \mu)\left[F\left(\frac{S-L \mu}{\sigma \sqrt{L}}\right)-F\left(\frac{S-(L+R) \mu}{\sigma \sqrt{L+R}}\right)\right]+R \mu F\left(\frac{S-(L+R) \mu}{\sigma \sqrt{L+R}}\right)+L \mu F\left(\frac{-L \mu}{\sigma \sqrt{L}}\right)-(L+
\end{aligned}
$$

R) $\left.\mu F\left(\frac{-(L+R) \mu}{\sigma \sqrt{L+R}}\right)\right\}$. Rearranging the terms, one obtains:

$$
\begin{aligned}
& \beta_{Z Z}=\frac{1}{R \mu}\left\{-\sigma \sqrt{L+R} f\left(\frac{S-(L+R) \mu}{\sigma \sqrt{L+R}}\right)-(S-L \mu) F\left(\frac{S-(L+R) \mu}{\sigma \sqrt{L+R}}\right)+R \mu F\left(\frac{S-(L+R) \mu}{\sigma \sqrt{L+R}}\right)\right\}+ \\
& \frac{1}{R \mu}\left\{\sigma \sqrt{L}\left[f\left(\frac{S-L \mu}{\sigma \sqrt{L}}\right)-f\left(\frac{-L \mu}{\sigma \sqrt{L}}\right)\right]+\sigma \sqrt{L+R} f\left(\frac{-(L+R) \mu}{\sigma \sqrt{L+R}}\right)+L \mu F\left(\frac{-L \mu}{\sigma \sqrt{L}}\right)-\right. \\
& \left.(L+R) \mu F\left(\frac{-(L+R) \mu}{\sigma \sqrt{L+R}}\right)+(S-L \mu) F\left(\frac{S-L \mu}{\sigma \sqrt{L}}\right)\right\}
\end{aligned}
$$

Note that for a standard normal distribution $f\left(\frac{-L \mu}{\sigma \sqrt{L}}\right) \cong 0, f\left(\frac{-(L+R) \mu}{\sigma \sqrt{L+R}}\right) \cong 0, F\left(\frac{-L \mu}{\sigma \sqrt{L}}\right) \cong$ $0, F\left(\frac{-(L+R) \mu}{\sigma \sqrt{L+R}}\right) \cong 0$. Thus, dropping the four related terms from (3-25) yields the following:

$$
\begin{aligned}
& \beta_{Z Z A p p^{\prime}}=\frac{1}{R \mu}\left\{-\sigma \sqrt{L+R} f\left(\frac{S-(L+R) \mu}{\sigma \sqrt{L+R}}\right)-(S-L \mu) F\left(\frac{S-(L+R) \mu}{\sigma \sqrt{L+R}}\right)+R \mu F\left(\frac{S-(L+R) \mu}{\sigma \sqrt{L+R}}\right)\right\}+ \\
& \frac{1}{R \mu}\left\{\sigma \sqrt{L} f\left(\frac{S-L \mu}{\sigma \sqrt{L}}\right)+(S-L \mu) F\left(\frac{S-L \mu}{\sigma \sqrt{L}}\right)\right\}
\end{aligned}
$$


Observe that Equation (3-26) is essentially the Silver and Bischak (2011) exact fill rate expression in Equation (3-24), thus one have $\beta_{Z Z A p p^{\prime}}=\beta_{S B}$. In words, $\beta_{S B}$ can be obtained from dropping several terms from Zhang and Zhang's exact formula $\beta_{Z Z}$.

Compare $\beta_{S B}$ in (3-24) (or equivalently (3-26)) and $\beta_{Z Z}$ in (3-25), if $-\sigma \sqrt{L} f\left(\frac{-L \mu}{\sigma \sqrt{L}}\right)+$ $\sigma \sqrt{L+R} f\left(\frac{-(L+R) \mu}{\sigma \sqrt{L+R}}\right)+L \mu F\left(\frac{-L \mu}{\sigma \sqrt{L}}\right)-(L+R) \mu F\left(\frac{-(L+R) \mu}{\sigma \sqrt{L+R}}\right) \geq 0$, then $\beta_{Z Z} \geq \beta_{S B}$

Lemma 3.2. If $\sigma \sqrt{L} f\left(\frac{S-L \mu}{\sqrt{L} \sigma}\right)+(S-L \mu) F\left(\frac{S-L \mu}{\sqrt{L} \sigma}\right) \geq(S-L \mu)$, then $\beta_{S B} \geq \beta_{Z Z a p p}$.

Proof. Further simplify (3-26) by deeming $f\left(\frac{S-L \mu}{\sigma \sqrt{L}}\right) \cong 0$ and $F\left(\frac{S-L \mu}{\sigma \sqrt{L}}\right) \cong 1$, then one obtains:

$\beta_{Z Z \text { App }}=\frac{1}{R \mu}\left\{-\sigma \sqrt{L+R} f\left(\frac{S-(L+R) \mu}{\sigma \sqrt{L+R}}\right)-(S-L \mu) F\left(\frac{S-(L+R) \mu}{\sigma \sqrt{L+R}}\right)+R \mu F\left(\frac{S-(L+R) \mu}{\sigma \sqrt{L+R}}\right)\right\}+$

$\frac{S-L \mu}{R \mu}$

In fact, (3-27) is Zhang and Zhang's approximation as in (3-23a).

Compare (3-26) to (3-27) (or equivalent (3-23a)), if $\sigma \sqrt{L} f\left(\frac{S-L \mu}{\sqrt{L} \mu}\right)+(S-L \mu) F\left(\frac{S-L \mu}{\sqrt{L} \mu}\right) \geq$ $(S-L \mu)$, then $\beta_{S B} \geq \beta_{Z Z A p p .}$

Theorem 3.3. If the following two conditions hold,

$$
\begin{aligned}
& \text { (i) } \quad-\sigma \sqrt{L} f\left(\frac{-L \mu}{\sigma \sqrt{L}}\right)+\sigma \sqrt{L+R} f\left(\frac{-(L+R) \mu}{\sigma \sqrt{L+R}}\right)+L \mu F\left(\frac{-L \mu}{\sigma \sqrt{L}}\right)-(L+R) \mu F\left(\frac{-(L+R) \mu}{\sigma \sqrt{L+R}}\right) \geq 0 ; \\
& \text { (ii) } \quad \sigma \sqrt{L} f\left(\frac{S-L \mu}{\sigma \sqrt{L}}\right)+(S-L \mu) F\left(\frac{S-L \mu}{\sigma \sqrt{L}}\right) \geq(S-L \mu)
\end{aligned}
$$

then $\beta_{Z Z} \geq \beta_{S B} \geq \beta_{Z Z a p p}$. 
Proof. The result follows immediately from Lemmas 3.1 and 3.2.

Finally, we note that both conditions (i) and (ii) are fairly mild and they hold for almost all cases we have tested. As a result, our simulations support $\beta_{Z Z} \geq \beta_{S B} \geq \beta_{Z Z \text { App. }}$

\subsection{Scaling a General Periodic Review System}

Now we have demonstrated all the eight formulas covered in this thesis. However, among these eight formulas, only some of them can be used in a general periodic review system directly while others need to be implemented in a standard periodic review system. But in a realistic world, most of the companies adopt general periodic review system rather than standard periodic review system due to relative high review cost of standard periodic review system. Thus, in this thesis, we consider a general periodic review system with review interval $R=2$ and replenishment lead time $L=4$. In order to test all these formulas under same criterion, we need to scale the general periodic review system into standard periodic review system as:

Table 3-2: The Scaling Technique for General Periodic Review

\begin{tabular}{|l|l|l|}
\hline Parameters & General periodic review & Standard periodic review \\
\hline$R$ & system & system \\
\hline$L$ & 4 & 1 \\
\hline$\mu$ & 200 & 2 \\
\hline$\sigma$ & 100 & $200 * 2=400$ \\
\hline
\end{tabular}


Following this method, a general periodic review system can be easily converted to a standard periodic review system, and therefore we can implement all the formulas equivalently.

\subsection{Chapter Summary}

This chapter provides an overview of all the fill rate formulas to be evaluated in this thesis, which includes the origin of each expression, major derivations of each expression and the relationships among them. Table 3-1 below provides a more concise theoretical comparison for these eight formulas. In the next chapter, we will discuss the design and implementation of the Monte Carlo Simulation for a periodic review inventory system with the order up to policy. The Monte Carlo simulation results are then used as a basis for evaluating all formula involved in this thesis.

Table 3-3: Theoretical Comparison between All Formulas

\begin{tabular}{|c|c|c|c|c|c|c|}
\hline 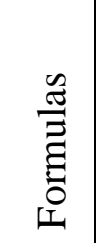 & 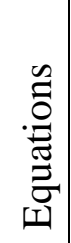 & Origins & 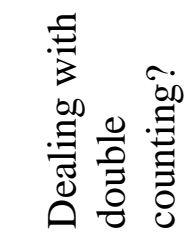 & $\begin{array}{l}\text { Distinctive } \\
\text { Ideas/features }\end{array}$ & $\begin{array}{l}\text { Adaption to the } \\
\text { general } \\
\text { Periodic review } \\
\text { system }\end{array}$ & $\begin{array}{l}\text { Relatio } \\
\text { ns with } \\
\text { others }\end{array}$ \\
\hline 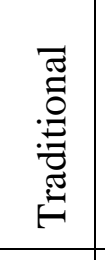 & $\begin{array}{c}\widehat{T} \\
\dot{c} \\
\tilde{c} \\
\widetilde{I}\end{array}$ & $\begin{array}{l}\text { calculate the } \\
\text { backorders } \\
\text { whenever } \\
\text { demands exceed } \\
\text { base stock level }\end{array}$ & No & $\begin{array}{l}\text { Easily } \\
\text { understand }\end{array}$ & $\mu^{\prime}=R^{*} \mu$ & \\
\hline 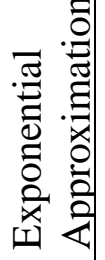 & 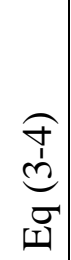 & $\begin{array}{l}\text { Approximated } \\
\text { from Traditional } \\
\text { approach }\end{array}$ & No & $\begin{array}{l}\text { eliminate unit } \\
\text { normal loss } \\
\text { function }\end{array}$ & $\mu^{\prime}=R^{*} \mu$ & $\begin{array}{l}\text { Traditio } \\
\text { nal's } \\
\text { approxi } \\
\text { mation }\end{array}$ \\
\hline
\end{tabular}




\begin{tabular}{|c|c|c|c|c|c|c|}
\hline 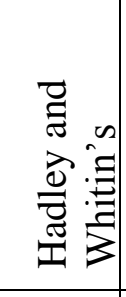 & $\begin{array}{c}6 \\
0 \\
1 \\
0 \\
0 \\
01\end{array}$ & $\begin{array}{l}\text { Calculate the } \\
\text { difference } \\
\text { between } \\
\text { backorders } \\
\text { reported in time } \\
(\mathrm{L}, \mathrm{R}+\mathrm{L})\end{array}$ & Yes & $\begin{array}{l}\text { Solve the } \\
\text { double } \\
\text { counting } \\
\text { problem }\end{array}$ & $\mu^{\prime}=R^{*} \mu$ & $\begin{array}{l}\text { Equival } \\
\text { ent to } \\
\text { Silver } \\
\text { and } \\
\text { Bischak } \\
(2011)\end{array}$ \\
\hline 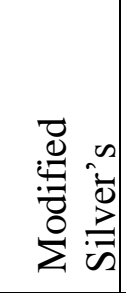 & 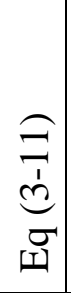 & $\begin{array}{l}\text { Truncate } \\
\text { inventory level } \\
\text { to three part, } \\
\text { calculate } \\
\text { backorders } \\
\text { respectively }\end{array}$ & Yes & $\begin{array}{l}\text { Solve the } \\
\text { double } \\
\text { counting } \\
\text { problem }\end{array}$ & $\begin{array}{l}\text { Scaling as in } \\
\text { Table 3-2 }\end{array}$ & \\
\hline $\begin{array}{l}n \\
\cdots \\
\pi \\
0 \\
0 \\
0 \\
0 \\
\vdots \\
0 \\
0\end{array}$ & 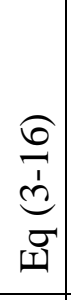 & $\begin{array}{l}\text { Truncated } \\
\text { inventory level } \\
\text { to two part, } \\
\text { calculate } \\
\text { backorders } \\
\text { respectively }\end{array}$ & Yes & $\begin{array}{l}\text { Solve the } \\
\text { double } \\
\text { counting } \\
\text { problem }\end{array}$ & $\begin{array}{l}\text { Scaling as in } \\
\text { Table 3-2 }\end{array}$ & \\
\hline 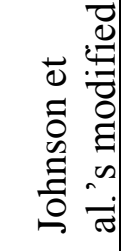 & 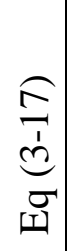 & $\begin{array}{l}\text { Eliminating } \\
\text { negative } \\
\text { demands from } \\
\text { total demands }\end{array}$ & Yes & $\begin{array}{l}\text { Can handle } \\
\text { demands of } \\
\text { high } \\
\text { variability }\end{array}$ & $\begin{array}{l}\text { Scaling as in } \\
\text { Table 3-2 }\end{array}$ & \\
\hline 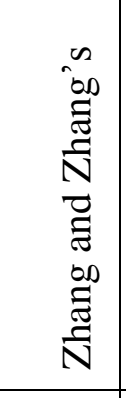 & 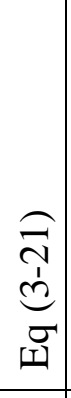 & $\begin{array}{l}\text { Derived from } \\
\text { the long run } \\
\text { fraction of } \\
\text { demand satisfied } \\
\text { from on hand } \\
\text { inventory }\end{array}$ & Yes & $\begin{array}{l}\text { Can be used } \\
\text { not only for } \\
\text { standard } \\
\text { periodic } \\
\text { system but } \\
\text { also general } \\
\text { periodic } \\
\text { system }\end{array}$ & Use directly & \\
\hline 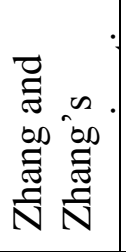 & 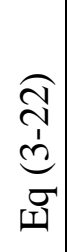 & $\begin{array}{l}\text { Approximated } \\
\text { based on the } \\
\text { properties of } \\
\text { normal } \\
\text { distribution }\end{array}$ & Yes & $\begin{array}{l}\text { Easily to } \\
\text { implement }\end{array}$ & Use directly & $\begin{array}{l}\text { Equival } \\
\text { ent to } \\
\text { Traditio } \\
\text { nal }\end{array}$ \\
\hline
\end{tabular}




\section{CHAPTER 4 DESIGN OF THE MONTE CARLO SIMULATION FOR SINGLE-STAGE INVENTORY SYSTEMS WITH PERIODIC}

\section{REVIEW AND BASE STOCK POLICY}

In this chapter, we discuss how the design of the Monte Carlo Simulation via MATLAB (MATLAB 2002) that imitates an inventory system under the periodic review and order up to policy. First, we discuss the inventory dynamics for the periodic review system based on a pre-determined chronology of events. Second, we will introduce the basic concepts and principles for the working of the Monte Carlo Simulation for inventory systems. Lastly, we briefly mention the random number generation with pseudo random number.

\subsection{Inventory Dynamics and Chronology of Events}

\section{Notations}

- $D_{t}$ denotes the demand in period $t$

- $S$ denotes the base stock level

- $I_{t b}$ denotes on hand inventory at the beginning of period $t$

- $I_{t e}$ denotes on hand inventory at the end of period $t$

- $Q_{t}$ denotes the replenishment order size placed in period $t$ 
In addition, the chronology, i.e., the sequence of events occurring in a particular period, is defined below. Note that if an inventory system follows a different chronology, the long run average nature of the $\beta$ service level implies that the system fill rate remains unchanged at any given time period $t$, the events occurs in the following order:

Firstly, previously placed replenishment order arrivals and will be added to the on hand inventory level immediately.

Secondly, if period $t$ is a review period, then the inventory level will be observed and another replenishment order will be placed if the physical inventory level (on hand inventory plus in-transit inventory) is lower than the base stock level $S$. If, on the other hand, the current period $t$ is not a review period or the physical inventory level exceeds the base stock level, then no replenishment order will be placed.

Lastly, demands for period $t$ will be realized. If the amount of on hand inventory is larger than the amount of the period t's demand, then all this demand will be satisfied. If, however, the on hand inventory is less than period t's demands, then only part of the demand is satisfied and the remaining will be backlogged. Finally, if the on hand inventory is already negative, then all period $t$ 's demand will be backlogged. Accordingly, the inventory dynamics are as follows:

$$
\begin{aligned}
& x_{(t-1) e}+Q_{t}=x_{t b} \\
& Q_{t}=S-x_{t b}-\sum_{i=0}^{t} Q_{i}, \text { where } t=n * R, \text { for any integer } n \\
& x_{t b}-x_{t e}=D_{t}
\end{aligned}
$$


In the next section, we will discuss how we design the Monte Carlo simulation to imitate the above inventory dynamics.

\subsection{Monte Carlo Simulation}

Monte Carlo Simulation, also as known as Monte Carlo method, is invented by Stanislaw Ulam in late 1940s while he was working on nuclear weapon projects at the Los Alamos National Laboratory (see, e.g., Anderson, 1987). Monte Carlo Simulation is a computational algorithm that obtains numerical results based on repeated random sampling. Sawilowsky (2003) notes that a high quality Monte Carlo Simulation must include the following characteristics:

- The pseudo random number generator has certain characteristics;

- The pseudo random number generator produces values that pass the test of randomness;

- There are enough samples to ensure accurate results;

- The proper sampling technique is used;

- The algorithm used is valid for what is modeled;

- The simulation simulates the phenomenon in question.

The Monte Carlo simulation in this thesis treats the periodic demand as the random variable, which follows normal distributions with constant mean and variance. We implement this using the "normrnd $(\mu, \sigma)$ " command in MATLAB (MATLAB 2002) with pseudo random seeds. The procedure of simulating these random demands and the afore-mentioned chronology of event are discussed below. 
First, we create an original matrix including, demand for each period, and beginning and ending "stock on hand" for each period, where the ending stock on hand equals the beginning stock on hand minus the demand for each period. For a particular period $t$, if demand is less than or equal to the beginning stock on hand, then all of the demand is satisfied without any backorders. If, however, the period demand exceeds the beginning stock on hand beginning and the beginning stock on hand is positive, then this demand less the beginning stock on hand will be backlogged. If, in a third scenario, the beginning stock on hand is already negative before this period's demand arrivals, then all this period's demand is backlogged. Consequently, the ending stock on hand will be updated accordingly.

Furthermore, if $t=n * R$, i.e., the current period is a review period, then the inventory level will be assessed. If the on hand inventory plus the in-transit inventory is smaller than the base stock level, an order will be placed.

Finally, the Monte Carlo simulation counts the total number of demands satisfied and the total number of demands over a long term (e.g., three years), and calculates the long run fraction of demands that are satisfied immediately by on-hand inventory as the simulated system $\beta$ service level. Our Monte Carlo simulation indeed simulates 156 weekly ( 3 year worth of length) inventories and omits the first 52 weeks of results, in order to calculate a more accurate long run fraction by excluding the transient period. Each scenario in the numerical experiments is simulated for 30 replications and the average of the 30 replications is reported as the $\beta$ service level for the corresponding scenario. 
In order to study the effect of various factors including review interval, lead time, demand variability and base stock level on the system fill rate, numerous scenarios were simulated. In particular, we use the $\alpha$ service level as the driver to vary the base stock level $S$ by employing the following equation:

$$
S=\mu(L+R)+Z_{\alpha} * \sigma \sqrt{L+R},
$$

where $Z_{\alpha}$ is a critical point such that $\operatorname{Prob}\left(Z \leq Z_{\alpha}\right)=\alpha$ for the standard normal variable $Z$. In other words,

$$
Z_{\alpha}=F^{-1}(\alpha)
$$

For each experimental scenario, we performed $m=30$ replications of the simulation, the period length of which is $n=153$ periods. In order to eliminate the large deviations from initial transient periods, we dropped the first $k=52$ periods as "warm up" periods from the final simulation analysis. For each replication of the simulation, we estimate the system's $\beta$ service level as follows:

$$
\beta_{j}=\frac{\sum_{t=k+1}^{n} D_{t}(\text { satisfied })}{\sum_{t=k+1}^{n} D_{t}} \text {, where } D_{t}(\text { satisfied }) \text { is the amount of demand satisfied }
$$
immediately by the on-hand inventory for period $t$.

Therefore, the final estimated fill rate from the Monte Carlo simulation is:

$$
\beta_{M C}=\frac{\sum_{j=1}^{m} \beta_{j}}{m}
$$

Finally, all random periodic demands are generated by using the "random number seeds" enabled in MATLAB (MATLAB 2002). Once the coefficient variance is pre- 
determined, all the period demands are determined as well. In this way, our Monte Carlo simulations are controllable and replicable. 


\section{CHAPTER 5 NUMERICAL RESULTS}

In this chapter, we report numerical results with two foci: the behavior of the $\beta$ service level for single-stage periodic review inventory system, and the comparison of accuracy for eight formulas documented in the literature and discussed extensively in Chapter 3. All the computational tests are implemented by MATLAB (MATLAB 2002) and Maple (Char 1988), and run on a Dell computer with Inter Pentinum R CPU 3.40 GHz and 3GB Ram.

The first part of this section is dedicated to observing the behavior of the $\beta$ service level under various settings of an inventory system. These include: 1) various base stock levels driven by a changing $\alpha$ service levels; 2) various combination of replenishment lead time $L$ and review interval $R$ such that $L+R$ is a constant; 3) various levels of demand variation driven by the so-called "coefficient of variation," i.e., $\mathrm{CV}=\frac{\sigma}{\mu}$. Lastly, numerical comparisons between "properly scaled" and "improperly scaled" inventory systems will show how the eight formulas adapt to general periodic review system.

Furthermore, for the cases with high demand variability, possible negative demand is a challenging issue inherited from the normal distribution. Under this circumstance, we perform two sets of Monte Carlo simulations. One accepts negative demand as it is, deeming these negative demands as "customer returns" as in Johnson et al. (1995). The other simulation, however, truncates all negative demands to zero, and 
calculates the system $\beta$ service level accordingly. These two sets of simulations are referred to as "MC results" and "Truncated MC results" respectively in this section.

The second part of this section focuses on comparison the accuracy of the eight formulas we reviewed in Chapter 3. The evaluation is based on the mean square error from the Monte Carlo simulation results, which is calculated as follows:

$$
\text { Mean Square Error }=\frac{1}{n} \sum_{i=1}^{n}(\mathrm{MC} \text { result }- \text { Expression result })^{2},
$$

where $n$ is the number of sample observations.

\subsection{Results for the behavior of the system $\beta$ fill rate}

Firstly, we create our baseline scenarios as: Lead Time $L=4$ weeks, Review interval $R=2$ weeks. Normally distributed period demand with mean $\mu=200$, standard deviation $\sigma=100$, and the corresponding base stock level $S=1200$. To create 50 experiments for high $\beta$ service level scenarios, we vary $\alpha$ service level from 0.5 to 0.99 ; to create 42 experiments for low $\beta$ service level scenarios, we vary $\alpha$ service level from 0.0015 to 0.5 . Lastly, we also vary lead time and review interval respectively to see how they affect the $\beta$ beta fill rate. All of our results from Monte Carlo simulations show that as base stock level $S$ increases, the corresponding $\beta$ service level increases, the expected shortage units trend to be decreased as more units of goods holding on hand, however, this would obviously increase the inventory holding cost and it requires the inventory manager to make a trade-off between high $\beta$ service level and high inventory holding cost.

Figure 5-1 shows that the $\alpha$ service level changes slightly more rapidly than the $\beta$ service level for the same increment of base stock level. This means that the $\alpha$ service 
level, an event-based measure, is more sensitive to the change of base stock level, than the $\beta$ service level, which is a quantity-based measure.

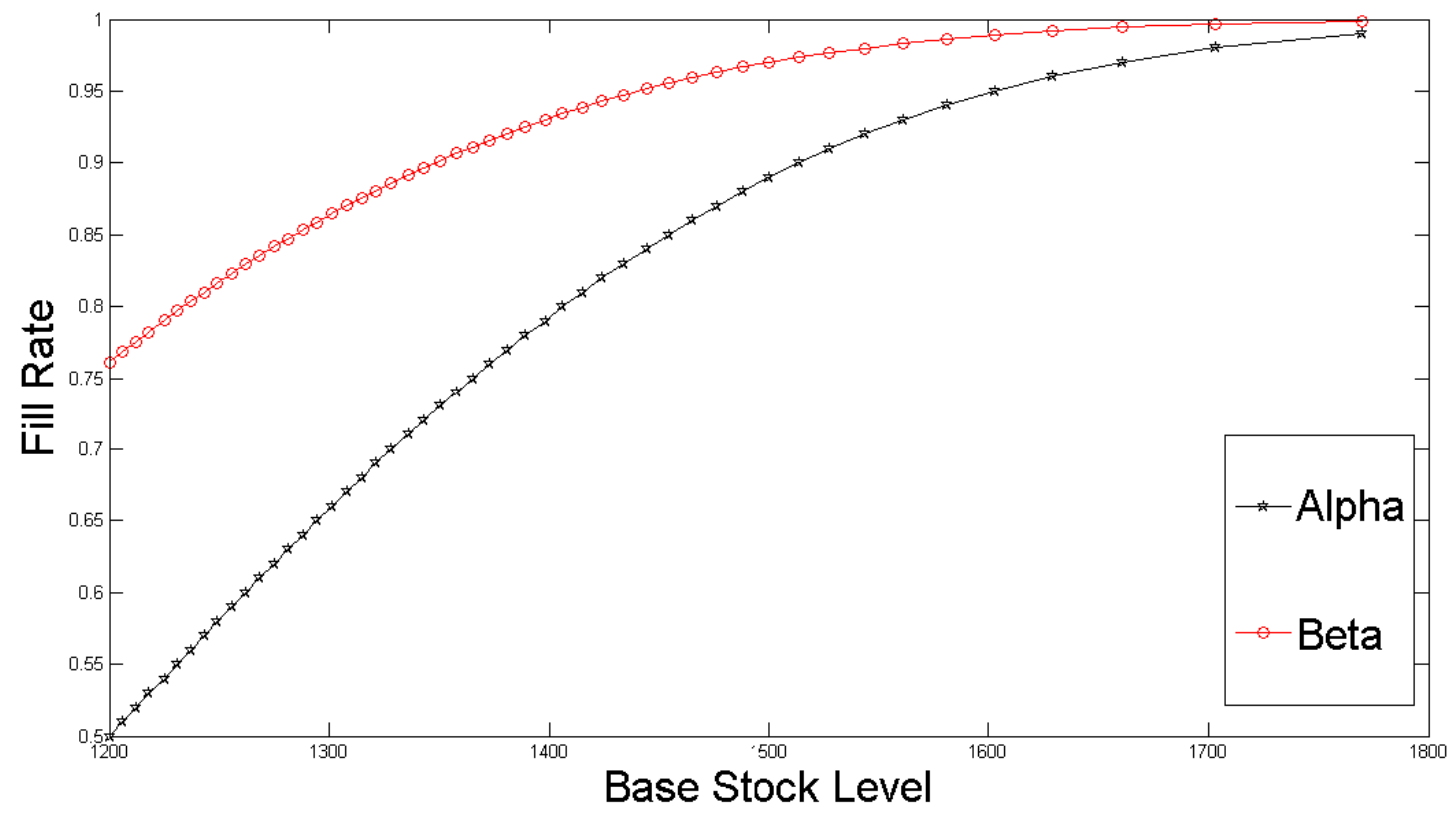

Figure 5-1: Alpha Fill Rate versus Beta Fill Rate

Table 5-1: $\beta$ service level vs. Review Interval $R$

\begin{tabular}{|c|c|c|c|c|c|}
\hline$S$ & $\mathrm{~L}$ & $\mathrm{R}$ & $\mu$ & $\sigma$ & $\beta$ service level \\
\hline 3000 & 10 & 1 & 200 & 60 & 1.0000 \\
\hline 3000 & 10 & 2 & 200 & 60 & 0.9999 \\
\hline 3000 & 10 & 3 & 200 & 60 & 0.9960 \\
\hline 3000 & 10 & 4 & 200 & 60 & 0.9714 \\
\hline 3000 & 10 & 5 & 200 & 60 & 0.9076 \\
\hline 3000 & 10 & 6 & 200 & 60 & 0.8211 \\
\hline 3000 & 10 & 7 & 200 & 60 & 0.7250 \\
\hline 3000 & 10 & 8 & 200 & 60 & 0.6338 \\
\hline
\end{tabular}




\begin{tabular}{|c|c|c|c|c|c|}
\hline 3000 & 10 & 9 & 200 & 60 & 0.5550 \\
\hline 3000 & 10 & 10 & 200 & 60 & 0.5179 \\
\hline
\end{tabular}

Table 5-2: $\beta$ service level vs. lead time $L$

\begin{tabular}{|c|c|c|c|c|c|}
\hline S & $\mathrm{L}$ & $\mathrm{R}$ & $\mu$ & $\sigma$ & $\beta$ service level \\
\hline 3000 & 1 & 10 & 200 & 60 & 1.0000 \\
\hline 3000 & 2 & 10 & 200 & 60 & 0.9999 \\
\hline 3000 & 3 & 10 & 200 & 60 & 0.9986 \\
\hline 3000 & 4 & 10 & 200 & 60 & 0.9879 \\
\hline 3000 & 5 & 10 & 200 & 60 & 0.9501 \\
\hline 3000 & 6 & 10 & 200 & 60 & 0.8807 \\
\hline 3000 & 7 & 10 & 200 & 60 & 0.7997 \\
\hline 3000 & 8 & 10 & 200 & 60 & 0.7067 \\
\hline 3000 & 9 & 10 & 200 & 60 & 0.6113 \\
\hline 3000 & 10 & 10 & 200 & 60 & 0.5179 \\
\hline
\end{tabular}




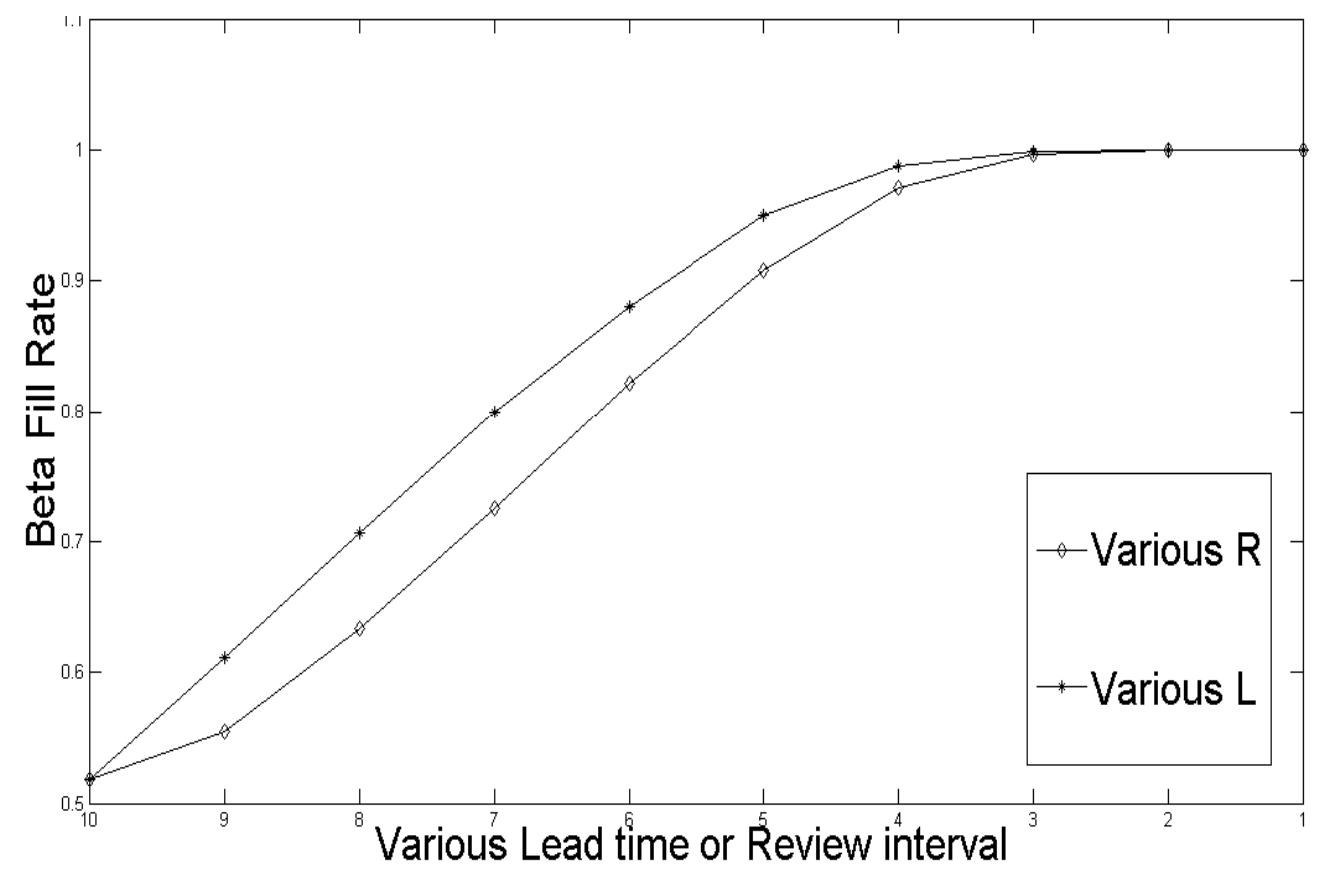

Figure 5-2 Various Lead Time versus Review Interval

Tables 5-1 and 5-2 summarize the results for the $\beta$ service level as we vary the review interval $R$ and the lead time $L$. Particularly, in Table $1 L$ is set to be a constant 10 , and $R$ varies from 1 to 10 . As a result, the system fill rate decreases from $100 \%$ to approximately $52 \%$. On the other hand, when $R$ is set to be a constant 10 as in Table 2, increasing the lead time $L$ from 1 to 10 results in the same reduction for the fill rate (from $100 \%$ to $52 \%$ ). This is because essentially the fill rate depends more on the value of $L+R$. However, Figure 5-2 provides more insights on which of the $L$ and $R$ has larger impact on the system fill rate. Clearly, from Figure 5-2, one observes the slope of the curve corresponding to varying the replenishment lead time is larger than that of the curve corresponding to varying the review interval. Thus, we conclude that the replenishment lead time has larger effect on the $\beta$ service level than does the review interval. 
Aside from the base stock level, replenishment lead time and review interval, coefficient of variation for the random demand also has a significant impact on the $\beta$ service level. As coefficient of periodic demands increase, $\beta$ service level decreases. Moreover, the $\alpha$ service level is lower than $\beta$ service level in general, but when the coefficient of variation is as high as 3 , it is very likely that the $\alpha$ service level is higher than the $\beta$ service level (see appendix for details). Table 5-3 below summarize the results for various $\mathrm{CV}$ under baseline scenario $L=4, R=2, \mu=200$. In order to detect how $\mathrm{CV}$ affect $\beta$ service level, we restrict the base stock levels constant and are equal to 1200 . From Table 3, we can see that as CV increase from 0.1 to 0.7 , the corresponding $\beta$ service level decrease from 0.9510 to 0.6792 , and this is because with high $\mathrm{CV}$, period demands trend to be more unstable. In other words, high CV will cause more stock out situations by producing more actual period demand larger than mean value of period demands.

Table 5-3: Various CV versus $\beta$ service level

\begin{tabular}{|c|c|c|c|c|c|c|c|}
\hline $\mathrm{cv}$ & $\mathrm{L}$ & $\mathrm{R}$ & $\mu$ & $\sigma$ & $\alpha$ & S & $\beta$ \\
\hline 0.1 & 4 & 2 & 200 & 20 & 0.5 & 1200 & 0.9510 \\
\hline 0.2 & 4 & 2 & 200 & 40 & 0.5 & 1200 & 0.9113 \\
\hline 0.3 & 4 & 2 & 200 & 60 & 0.5 & 1200 & 0.8557 \\
\hline 0.4 & 4 & 2 & 200 & 80 & 0.5 & 1200 & 0.8042 \\
\hline 0.5 & 4 & 2 & 200 & 100 & 0.5 & 1200 & 0.7613 \\
\hline 0.6 & 4 & 2 & 200 & 120 & 0.5 & 1200 & 0.7231 \\
\hline 0.7 & 4 & 2 & 200 & 140 & 0.5 & 1200 & 0.6792 \\
\hline
\end{tabular}




\subsection{Results for evaluation of the $\beta$ service level expressions}

\subsubsection{Performance comparison with High Fill Rate}

We first investigate the accuracy of all formulas in a relatively easy case where the system fill rate is medium to high, i.e., the $\beta$ service level is 0.7 or higher. Table 5-3 summarizes the results from the Monte Carlo simulation as well as the eight fill rate expressions, namely, traditional, exponential approximation, Hadley and Whitin's approach, Silver's modified approach, Johnson et al.'s approach, Johnson et al.'s modified approach, Zhang and Zhang, and Zhang and Zhang's approximation. The baseline scenario is again, $L=4, R=2, \mu=200$ and $\sigma=100$. In addition, we vary the $\alpha$ service level from 0.5 to 0.99 with an increment of 0.01 in order to obtain more sample points. Note that the resulting $\beta$ service level ranges from 0.76 to 0.999 . From Table 5-3, we observe that when base stock level $S$ change from 1200 to 1770 , our Monte Carlo simulation indicates system $\beta$ service level increase from 0.7613 to 0.9979 as well. In particular, all eight formulas can produce relatively accurate $\beta$ service level by comparing them with Monte Carlo simulation results. The mean square error, on the other hand, decreases in the sequence as: Exponential approximation, Traditional approach, Zhang and Zhang's approximation, Silver's modified, Johnson et al.'s modified approach, Johnson et al.'s approach, Hadley \& Whititn's, Zhang and Zhang's approach. Lastly, numerical results also indicate that traditional approach and Zhang and Zhang's approximation is equivalent to each other. 
Table 5-4: Performance Comparison with High Fill Rate $(\beta \geq 0.7)$

\begin{tabular}{|c|c|c|c|c|c|c|c|c|c|c|}
\hline$\alpha$ & S & MC result & Tradition & Exp & Hadley & Silver & J. et al. & J. et al. mod & $Z \& Z$ & $\mathrm{Z} \& \mathrm{Z}$ app \\
\hline 0.5 & 1200 & 0.7613 & 0.7557 & 0.7560 & 0.7599 & 0.7689 & 0.7600 & 0.7600 & 0.7599 & 0.7557 \\
\hline 0.51 & 1206 & 0.7684 & 0.7631 & 0.7630 & 0.7670 & 0.7756 & 0.7671 & 0.7671 & 0.7670 & 0.7631 \\
\hline 0.52 & 1212 & 0.7755 & 0.7704 & 0.7700 & 0.7740 & 0.7821 & 0.7741 & 0.7741 & 0.7740 & 0.7704 \\
\hline 0.53 & 1218 & 0.7824 & 0.7775 & 0.7768 & 0.7809 & 0.7886 & 0.7809 & 0.7809 & 0.7809 & 0.7775 \\
\hline 0.54 & 1225 & 0.7904 & 0.7857 & 0.7847 & 0.7887 & 0.7960 & 0.7887 & 0.7888 & 0.7887 & 0.7857 \\
\hline 0.55 & 1231 & 0.7971 & 0.7925 & 0.7913 & 0.7953 & 0.8022 & 0.7953 & 0.7954 & 0.7953 & 0.7925 \\
\hline 0.56 & 1237 & 0.8037 & 0.7992 & 0.7978 & 0.8017 & 0.8083 & 0.8018 & 0.8018 & 0.8017 & 0.7992 \\
\hline 0.57 & 1243 & 0.8101 & 0.8057 & 0.8042 & 0.8080 & 0.8143 & 0.8081 & 0.8081 & 0.8080 & 0.8057 \\
\hline 0.58 & 1249 & 0.8163 & 0.8121 & 0.8105 & 0.8142 & 0.8201 & 0.8143 & 0.8143 & 0.8142 & 0.8121 \\
\hline 0.59 & 1256 & 0.8234 & 0.8193 & 0.8176 & 0.8213 & 0.8268 & 0.8213 & 0.8214 & 0.8213 & 0.8193 \\
\hline 0.6 & 1262 & 0.8293 & 0.8254 & 0.8237 & 0.8272 & 0.8325 & 0.8272 & 0.8273 & 0.8272 & 0.8254 \\
\hline 0.61 & 1268 & 0.8351 & 0.8313 & 0.8295 & 0.8330 & 0.8380 & 0.8330 & 0.8330 & 0.8330 & 0.8313 \\
\hline 0.62 & 1275 & 0.8417 & 0.8381 & 0.8363 & 0.8396 & 0.8442 & 0.8396 & 0.8396 & 0.8395 & 0.8381 \\
\hline 0.63 & 1281 & 0.8473 & 0.8437 & 0.8419 & 0.8451 & 0.8495 & 0.8451 & 0.8451 & 0.8450 & 0.8437 \\
\hline 0.64 & 1288 & 0.8535 & 0.8501 & 0.8483 & 0.8513 & 0.8554 & 0.8513 & 0.8514 & 0.8513 & 0.8501 \\
\hline 0.65 & 1294 & 0.8587 & 0.8554 & 0.8536 & 0.8565 & 0.8604 & 0.8566 & 0.8566 & 0.8565 & 0.8554 \\
\hline 0.66 & 1301 & 0.8646 & 0.8615 & 0.8597 & 0.8625 & 0.8661 & 0.8625 & 0.8625 & 0.8625 & 0.8615 \\
\hline 0.67 & 1308 & 0.8703 & 0.8673 & 0.8656 & 0.8682 & 0.8716 & 0.8682 & 0.8683 & 0.8682 & 0.8673 \\
\hline 0.68 & 1315 & 0.8759 & 0.8730 & 0.8713 & 0.8738 & 0.8770 & 0.8738 & 0.8739 & 0.8738 & 0.8730 \\
\hline 0.69 & 1321 & 0.8806 & 0.8777 & 0.8761 & 0.8785 & 0.8814 & 0.8785 & 0.8785 & 0.8785 & 0.8777 \\
\hline
\end{tabular}




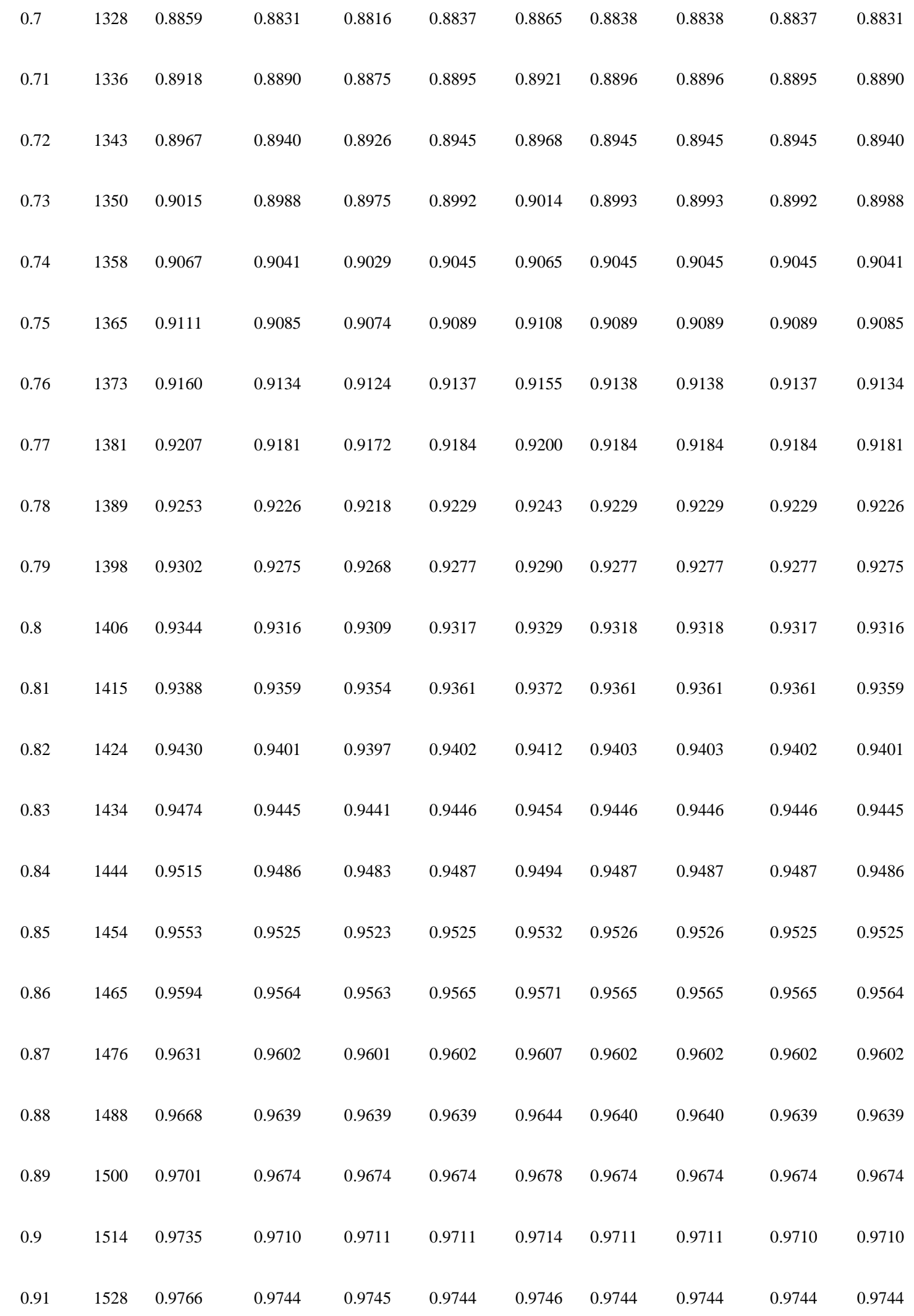




\begin{tabular}{|c|c|c|c|c|c|c|c|c|c|c|}
\hline 0.92 & 1544 & 0.9798 & 0.9778 & 0.9779 & 0.9778 & 0.9780 & 0.9778 & 0.9778 & 0.9778 & 0.9778 \\
\hline 0.93 & 1561 & 0.9829 & 0.9810 & 0.9811 & 0.9810 & 0.9811 & 0.9810 & 0.9810 & 0.9810 & 0.9810 \\
\hline 0.94 & 1581 & 0.9860 & 0.9842 & 0.9843 & 0.9842 & 0.9843 & 0.9842 & 0.9842 & 0.9842 & 0.9842 \\
\hline 0.95 & 1603 & 0.9890 & 0.9872 & 0.9873 & 0.9872 & 0.9873 & 0.9872 & 0.9872 & 0.9872 & 0.9872 \\
\hline 0.96 & 1629 & 0.9918 & 0.9901 & 0.9902 & 0.9901 & 0.9902 & 0.9901 & 0.9901 & 0.9901 & 0.9901 \\
\hline 0.97 & 1661 & 0.9944 & 0.9929 & 0.9930 & 0.9929 & 0.9929 & 0.9929 & 0.9929 & 0.9929 & 0.9929 \\
\hline 0.98 & 1703 & 0.9967 & 0.9955 & 0.9955 & 0.9955 & 0.9955 & 0.9955 & 0.9955 & 0.9955 & 0.9955 \\
\hline 0.99 & 1770 & 0.9990 & 0.9979 & 0.9979 & 0.9979 & 0.9979 & 0.9979 & 0.9979 & 0.9979 & 0.9979 \\
\hline & & MSE & $1.07 * 10^{-5}$ & $1.75 * 10^{-5}$ & $4.91 * 10^{-5}$ & $8.77 * 10^{-5}$ & $4.79 * 10^{-5}$ & $4.69 * 10^{-5}$ & $4.92 * 10^{-5}$ & $1.07 * 10^{-5}$ \\
\hline
\end{tabular}

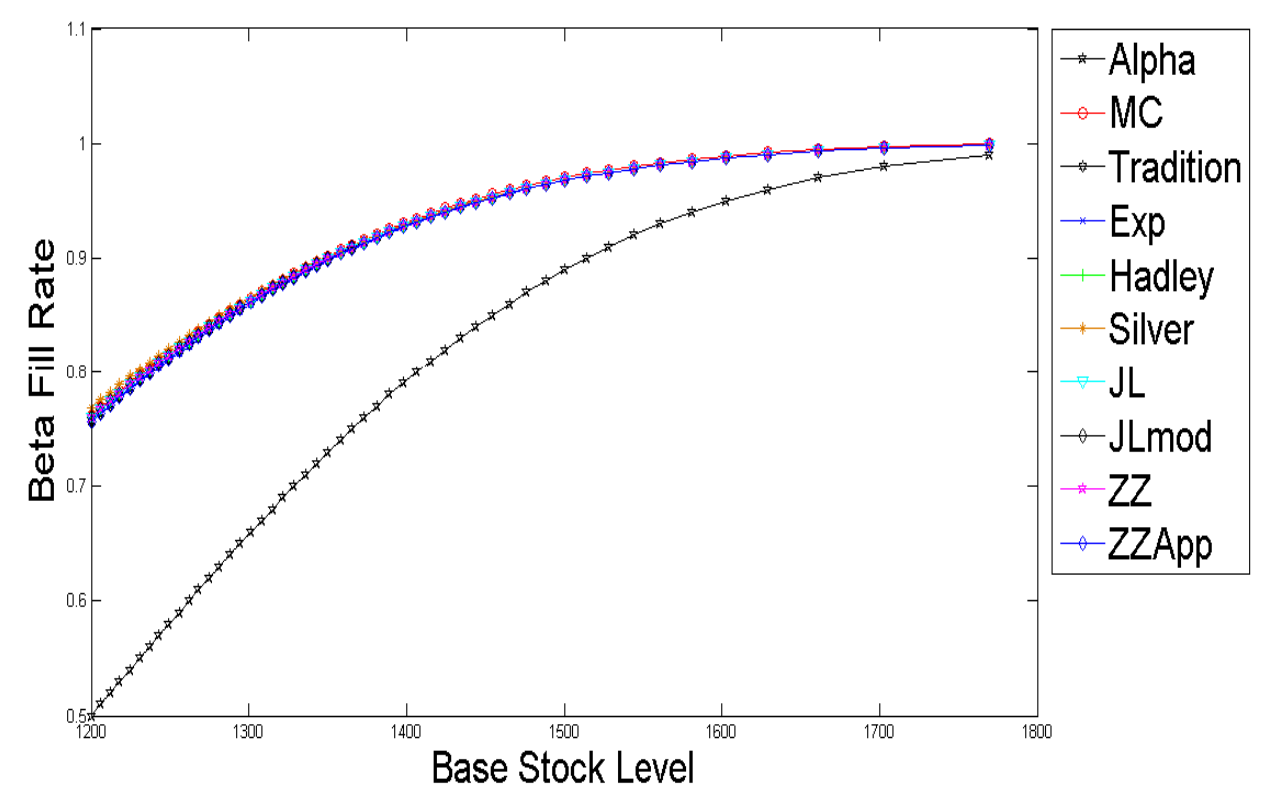

Figure 5-3: MC results versus Computation Results with High fill Rate 


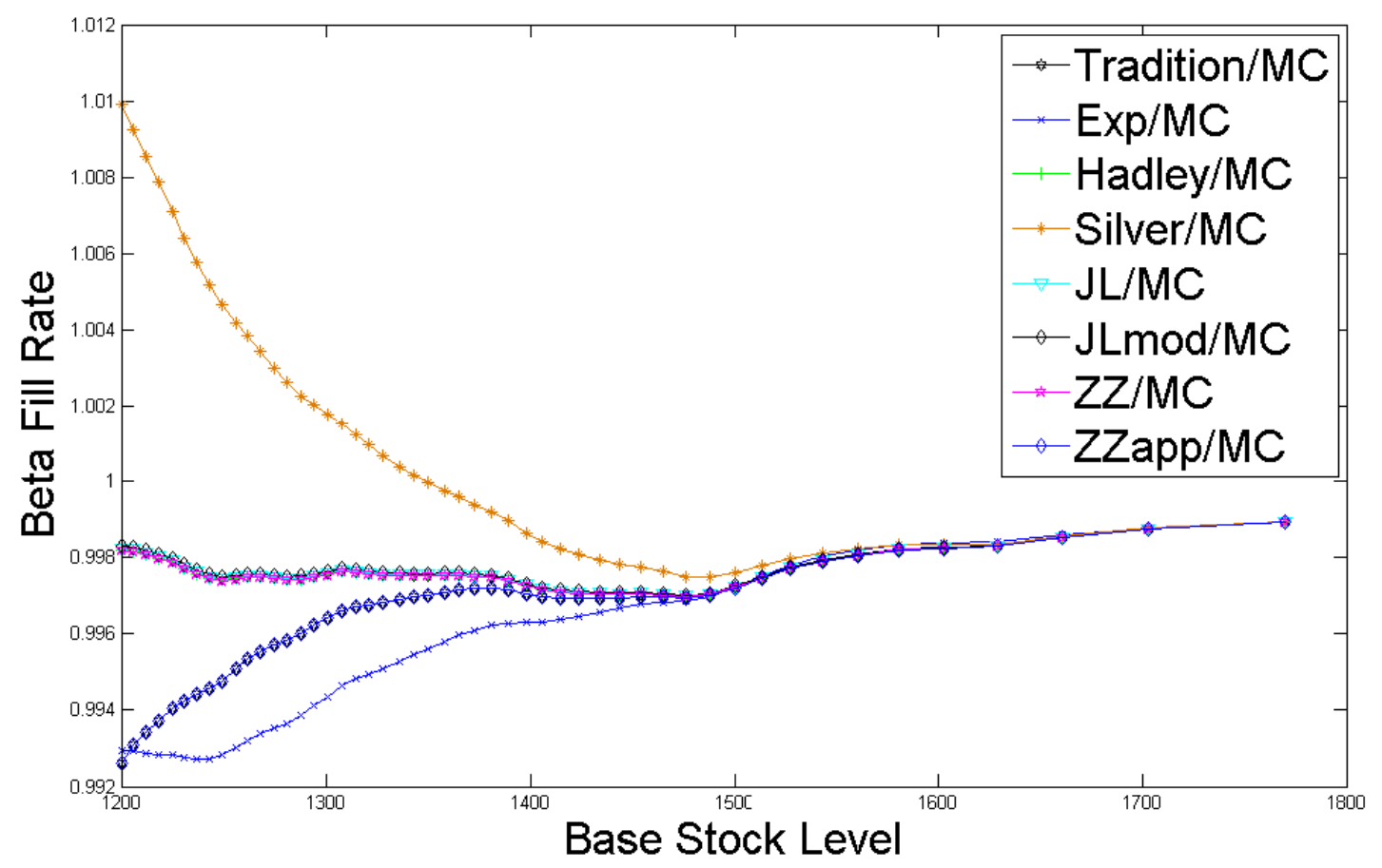

Figure 5-4: plot for Computation Results over MC results

Figure 5-3 displays the performances for all the formulas when the $\alpha$ service level increases from 0.5 to 0.99 . From this figure, one observes that all the formulas perform well because they all produced a fill rate that matches with the MC simulation. From a slightly different perspective, Figure 5-4 calculates the ratio between the fill rate produced by a formula and by the Monte Carlo simulation, i.e., $r_{i}=\frac{\beta_{i}}{\beta_{M C}}$. Thus, if $r_{i}>1$ then the corresponding formula $\beta_{i}$ overestimates the system fill rate, and vice versa. From Figure 5-4, Silver's approach tends to overestimate the $\beta$ service level when the base stock level is low, and starts to underestimate when the base stock level is high. On the other hand, traditional approach and the exponential approximation underestimate the system's $\beta$ service level. Furthermore, Hadley and Whitin's, Johnson et al. and Zhang 
and Zhang's approaches only underestimate the $\beta$ service level very slightly, and they dominate all other formulas in terms of accuracy.

\subsubsection{Performance comparison with Low Fill Rate}

We now examine the performance of all formulas when the system's $\beta$ service level is relatively low, i.e., 0.7 or below. We achieve this by varying the $\alpha$ service level from 0.0015 to 0.5 , thus varying the base stock level accordingly. Table 5-4 summarizes the results for the Monte Carlo simulation as well as the eight fill rate expressions when $L=4$, $R=2, \mu=200$ and $\sigma=100$. As shown in Table 5-4, when the $\alpha$ service level changes from 0.0015 to 0.5 , the $\beta$ service level changes from 0.009 to 0.76 , respectively. Furthermore, Table 5-4 shows that only Hadley and Whitin's approach, Johnson et al.'s approach, Johnson et al.'s modified approach, Zhang and Zhang's approach provide accurate results, with the mean square error values of $1.15^{*} 10^{-6}, 1.16^{*} 10^{-6}, 1.25^{*} 10^{-6}, 1.14^{*} 10^{-6}$, respectively. Also, one observes that when the base stock level is low, traditional approach, and Zhang and Zhang's approximation produce negative values. This is because sufficiently small base stock level would make the "double counting" even worse and produce more shortage than the total demand. Thus, these two approaches would provide negative $\beta$ service level, which is wrong.

Table 5-5: Performance Comparison with Low Fill Rate $(\beta \leq 0.7)$

\begin{tabular}{lcccccccccc}
\hline$\alpha$ & S & MC result & Tradition & Exp & Hadley & Silver & J.et al. & J. et al. mod $Z \& Z$ & Z\&Z app \\
\hline 0.0015 & 473 & 0.0091 & negative & 0.6797 & 0.0104 & 0.0256 & 0.0104 & 0.0104 & 0.0104 & negative \\
0.002 & 495 & 0.0122 & negative & 0.6504 & 0.0135 & 0.0309 & 0.0135 & 0.0135 & 0.0135 & negative \\
0.0025 & 512 & 0.0151 & negative & 0.6275 & 0.0163 & 0.0356 & 0.0163 & 0.0163 & 0.0163 & negative \\
\hline
\end{tabular}




\begin{tabular}{|c|c|c|c|c|c|c|c|c|c|c|}
\hline 0.003 & 527 & 0.0182 & negative & 0.6072 & 0.0192 & 0.0403 & 0.0192 & 0.0193 & 0.0192 & negative \\
\hline 0.0035 & 539 & 0.0211 & negative & 0.5910 & 0.0219 & 0.0443 & 0.0219 & 0.0219 & 0.0219 & negative \\
\hline 0.004 & 550 & 0.0239 & negative & 0.5762 & 0.0245 & 0.0483 & 0.0245 & 0.0246 & 0.0245 & negative \\
\hline 0.0045 & 560 & 0.0266 & negative & 0.5629 & 0.0272 & 0.0521 & 0.0272 & 0.0272 & 0.0272 & negative \\
\hline 0.005 & 569 & 0.0291 & negative & 0.5510 & 0.0298 & 0.0558 & 0.0298 & 0.0298 & 0.0298 & negative \\
\hline 0.0055 & 577 & 0.0316 & negative & 0.5405 & 0.0322 & 0.0592 & 0.0322 & 0.0323 & 0.0322 & negative \\
\hline 0.006 & 585 & 0.0342 & negative & 0.5302 & 0.0348 & 0.0628 & 0.0349 & 0.0349 & 0.0348 & negative \\
\hline 0.0065 & 592 & 0.0366 & negative & 0.5213 & 0.0373 & 0.0661 & 0.0373 & 0.0373 & 0.0373 & negative \\
\hline 0.0075 & 604 & 0.0411 & negative & 0.5063 & 0.0417 & 0.0721 & 0.0418 & 0.0418 & 0.0417 & negative \\
\hline 0.008 & 610 & 0.0436 & negative & 0.4990 & 0.0441 & 0.0752 & 0.0442 & 0.0442 & 0.0441 & negative \\
\hline 0.0085 & 615 & 0.0458 & negative & 0.4929 & 0.0462 & 0.0778 & 0.0462 & 0.0463 & 0.0462 & negative \\
\hline 0.009 & 621 & 0.0485 & negative & 0.4858 & 0.0488 & 0.0811 & 0.0488 & 0.0489 & 0.0488 & negative \\
\hline 0.01 & 630 & 0.0528 & negative & 0.4754 & 0.0529 & 0.0863 & 0.0529 & 0.0529 & 0.0529 & negative \\
\hline 0.015 & 668 & 0.0566 & negative & 0.4664 & 0.0568 & 0.0911 & 0.0568 & 0.0568 & 0.0567 & negative \\
\hline 0.02 & 697 & 0.0926 & negative & 0.4098 & 0.0921 & 0.1323 & 0.0921 & 0.0922 & 0.0921 & negative \\
\hline 0.025 & 720 & 0.1095 & negative & 0.3932 & 0.1094 & 0.1514 & 0.1094 & 0.1095 & 0.1094 & negative \\
\hline 0.03 & 739 & 0.1255 & negative & 0.3822 & 0.1253 & 0.1685 & 0.1253 & 0.1254 & 0.1253 & negative \\
\hline 0.035 & 756 & 0.1410 & negative & 0.3745 & 0.1408 & 0.1847 & 0.1408 & 0.1409 & 0.1408 & negative \\
\hline 0.04 & 771 & 0.1552 & negative & 0.3696 & 0.1554 & 0.1999 & 0.1554 & 0.1555 & 0.1554 & negative \\
\hline 0.045 & 785 & 0.1692 & negative & 0.3665 & 0.1699 & 0.2146 & 0.1699 & 0.1700 & 0.1699 & negative \\
\hline 0.05 & 797 & 0.1821 & negative & 0.3651 & 0.1829 & 0.2278 & 0.1830 & 0.1830 & 0.1829 & negative \\
\hline 0.055 & 809 & 0.1956 & 0.0082 & 0.3648 & 0.1965 & 0.2413 & 0.1966 & 0.1966 & 0.1965 & 0.0082 \\
\hline
\end{tabular}




\begin{tabular}{|c|c|c|c|c|c|c|c|c|c|c|}
\hline 0.06 & 819 & 0.2072 & 0.0317 & 0.3654 & 0.2083 & 0.2530 & 0.2083 & 0.2084 & 0.2083 & 0.0317 \\
\hline 0.065 & 829 & 0.2191 & 0.0552 & 0.3668 & 0.2204 & 0.2649 & 0.2205 & 0.2205 & 0.2204 & 0.0552 \\
\hline 0.07 & 839 & 0.2316 & 0.0785 & 0.3690 & 0.2329 & 0.2771 & 0.2330 & 0.2330 & 0.2329 & 0.0785 \\
\hline 0.075 & 847 & 0.2417 & 0.0970 & 0.3713 & 0.2432 & 0.2870 & 0.2432 & 0.2433 & 0.2432 & 0.0970 \\
\hline 0.08 & 856 & 0.2535 & 0.1178 & 0.3744 & 0.2550 & 0.2984 & 0.2550 & 0.2551 & 0.2550 & 0.1178 \\
\hline 0.085 & 864 & 0.2642 & 0.1361 & 0.3777 & 0.2657 & 0.3087 & 0.2657 & 0.2658 & 0.2657 & 0.1361 \\
\hline 0.09 & 872 & 0.2749 & 0.1544 & 0.3815 & 0.2766 & 0.3191 & 0.2766 & 0.2767 & 0.2766 & 0.1544 \\
\hline 0.095 & 879 & 0.2844 & 0.1703 & 0.3852 & 0.2863 & 0.3284 & 0.2863 & 0.2864 & 0.2863 & 0.1703 \\
\hline 0.1 & 886 & 0.2942 & 0.1860 & 0.3892 & 0.2962 & 0.3377 & 0.2962 & 0.2963 & 0.2961 & 0.1860 \\
\hline 0.15 & 946 & 0.3832 & 0.3175 & 0.4369 & 0.3853 & 0.4214 & 0.3854 & 0.3855 & 0.3853 & 0.3175 \\
\hline 0.2 & 994 & 0.4591 & 0.4166 & 0.4890 & 0.4607 & 0.4912 & 0.4607 & 0.4608 & 0.4607 & 0.4166 \\
\hline 0.25 & 1035 & 0.5243 & 0.4961 & 0.5401 & 0.5256 & 0.5511 & 0.5256 & 0.5257 & 0.5256 & 0.4961 \\
\hline 0.3 & 1072 & 0.5830 & 0.5631 & 0.5892 & 0.5831 & 0.6043 & 0.5831 & 0.5832 & 0.5831 & 0.5631 \\
\hline 0.35 & 1106 & 0.6349 & 0.6205 & 0.6352 & 0.6341 & 0.6515 & 0.6341 & 0.6342 & 0.6341 & 0.6205 \\
\hline 0.4 & 1138 & 0.6810 & 0.6705 & 0.6779 & 0.6798 & 0.6939 & 0.6798 & 0.6799 & 0.6798 & 0.6705 \\
\hline 0.45 & 1169 & 0.7227 & 0.7150 & 0.7180 & 0.7214 & 0.7327 & 0.7214 & 0.7215 & 0.7214 & 0.7150 \\
\hline \multirow[t]{2}{*}{0.5} & 1200 & 0.7613 & 0.7557 & 0.7560 & 0.7599 & 0.7689 & 0.7600 & 0.7600 & 0.7599 & 0.7557 \\
\hline & & MSE & & 0.1253 & $1.15^{*} 10^{-6}$ & 0.0012 & $1.16 * 10^{-6}$ & $1.25^{*} 10^{-6}$ & $1.14 * 10^{-6}$ & \\
\hline
\end{tabular}




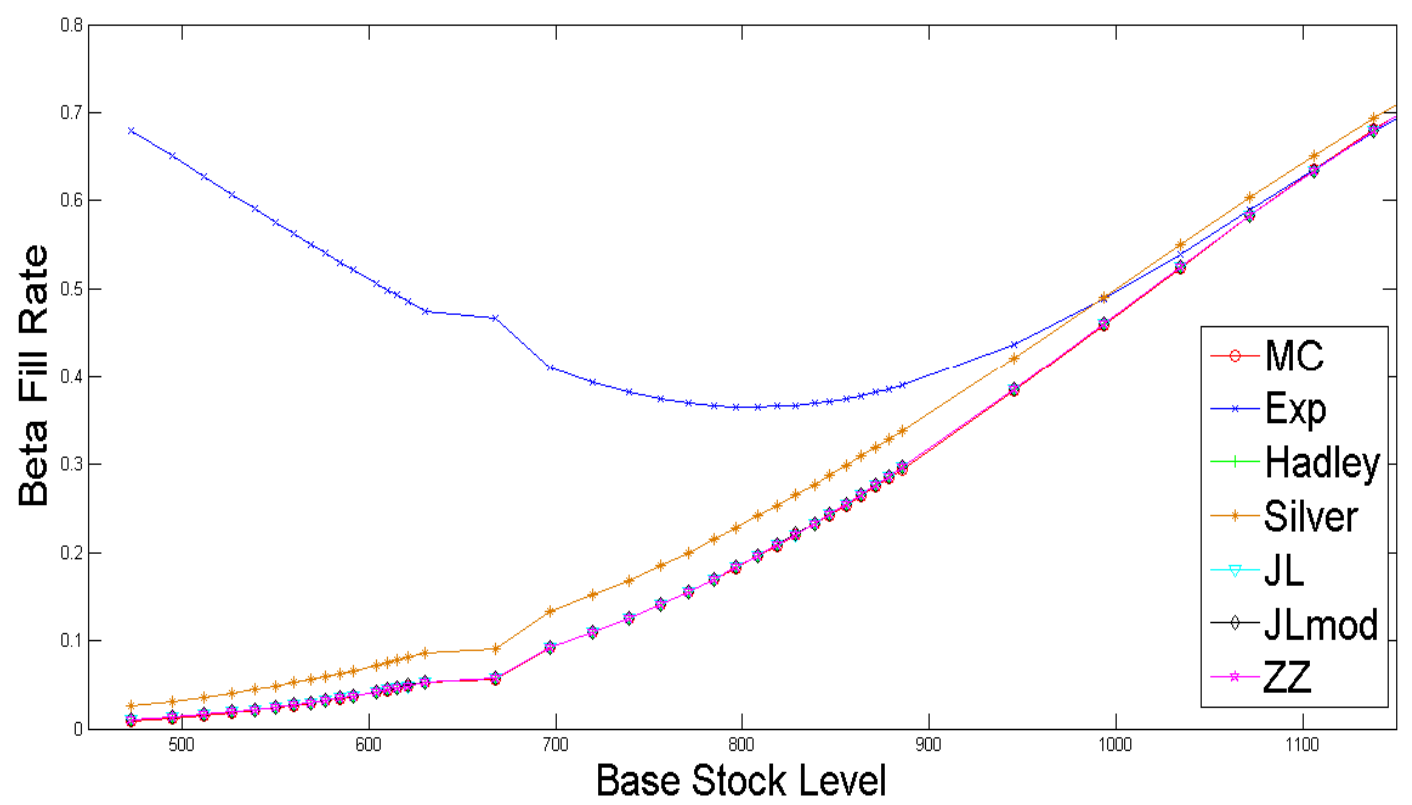

Figure 5-5: Performance Comparison with Low Fill Rate

In Figure 5-5, due to negative $\beta$ service level value produced by the traditional approach, and Zhang and Zhang's approximation, we exclude these two approaches from the our evaluation. From the figure, the exponential approximation performs the worst. In addition, Silver's approach again overestimates the system $\beta$ service level with relatively large discrepancy. It is concluded that when the system fill rate is low, Johnson et al.'s approach and Zhang and Zhang's method produce the most accurate results.

\subsubsection{Performance comparison when varying the $C V$}

After discussing the performance of the eight formulas (against the Monte Carlo simulation) when the system fill rate is either high or low in section 5.2.1 and 5.2.2, we now compare their performances against varying level of demand variation. In particular, we vary the coefficient of variation $(C V)$ of the normal random demand from 0.1 to 3 while keeping other system parameters $(L, R, \mu$ and $S)$ the same as in the baseline scenario. As mentioned previously, when negative demands occur due to high values of 
$\mathrm{CV}$, we present both Monte Carlo simulation as well as "truncated" Monte Carlo simulation. In the latter, negative demands are truncated to zero in order to resemble reallife situations. Tables S-1 through S-12 and Figure S-1 through S-12 in the Appendix summarize and plot all numerical results under various CVs.

Table 5-5 below summarizes all results in Tables S-1 through S-10 in an attempt to identify which method is more accurate, i.e., has smaller mean square error, for different $C V$ settings. The mean square error in Table 5-5 is calculated against the Monte Carlo simulation for CV from 0.1 to 3. Similarly, Table 5-6 summarizes the mean square error that compare all formulas against the "truncated" Monte Carlo simulation for CV from 0.8 to 3 (where negative demands becomes significant). Again, when a method (e.g., traditional, exponential approximation and Zhang and Zhang's approximation) produces negative $\beta$ service level, it is dropped from any further analysis. Table 5-5 displays that when $\mathrm{CV}$ is low, for instance, when $\mathrm{CV}=0.2$, the MSE for all eight formulas is as low as $5.08^{*} 10^{-5}$, except exponential approximation with a relative high MSE value of $3.15^{*} 10^{-5}$. As CV increases to 0.7, the MSE for Hadley and Whitin's approach, Johnson et al's approach and Johnson et al's modified approach and Zhang and Zhang's approach still remain low with values of $1.95 * 10^{-5}, 1.96 * 10^{-5}, 2.34 * 10^{-5}, 1.7 * 10^{-5}$, respectively. However, the MSE of other formulas increases significantly. Finally, when CV is 1 or higher, Johnson et al's modified approach has the lowest MSE of $3.58^{*} 10^{-5}$, performing better than Hadley and Whitin's, Johnson et al.'s original, and Zhang and Zhang's approaches.

Table 5-6: Mean Square Error For Various CV 


\begin{tabular}{|c|c|c|c|c|c|c|c|c|}
\hline$\triangle M C$ & Tradion & Exp & Hadley & Silver & J. et al. & $\begin{array}{l}\text { J. et al. } \\
\text { mod. }\end{array}$ & $Z \& Z$ & Z\&Z app \\
\hline 0.1 & $1.29 * 10^{-7}$ & $4.47 * 10^{-6}$ & $1.29 * 10^{-7}$ & $1.29 * 10^{-7}$ & $1.29 * 10^{-7}$ & $1.29 * 10^{-7}$ & $1.29 * 10^{-7}$ & $1.29 * 10^{-7}$ \\
\hline 0.2 & $5.08 * 10^{-5}$ & $3.15^{*} 10^{-5}$ & $5.08 * 10^{-5}$ & $5.08 * 10^{-5}$ & $5.08 * 10^{-5}$ & $5.08 * 10^{-5}$ & $5.08 * 10^{-5}$ & $5.08 * 10^{-5}$ \\
\hline 0.3 & $9.01 * 10^{-6}$ & $1.17 * 10^{-5}$ & $8.36^{*} 10^{-6}$ & $5.55^{*} 10^{-6}$ & $8.36^{*} 10^{-6}$ & $8.35 * 10^{-6}$ & $8.38 * 10^{-6}$ & $9.01 * 10^{-6}$ \\
\hline 0.4 & $4.56^{*} 10^{-6}$ & $3.39 * 10^{-5}$ & $8.05 * 10^{-7}$ & $1.62 * 10^{-5}$ & $8.05^{*} 10^{-7}$ & $8.06 * 10^{-7}$ & $7.8 * 10^{-7}$ & $4.56^{*} 10^{-7}$ \\
\hline 0.5 & $7.21 * 10^{-5}$ & $1.82 * 10^{-5}$ & $3.29 * 10^{-6}$ & $8.68 * 10^{-5}$ & $3.28 * 10^{-6}$ & $3.20 * 10^{-6}$ & $3.38 * 10^{-7}$ & $7.21 * 10^{-7}$ \\
\hline 0.6 & 0.0004 & 0.0001 & $1.42 * 10^{-5}$ & 0.0002 & $1.40 * 10^{-5}$ & $1.41 * 10^{-5}$ & $1.5^{*} 10^{-5}$ & 0.0004 \\
\hline 0.7 & 0.0012 & 0.0005 & $1.95 * 10^{-5}$ & 0.0007 & $1.96 * 10^{-5}$ & $2.34 * 10^{-5}$ & $1.7 * 10^{-5}$ & 0.0012 \\
\hline 0.8 & 0.0045 & 0.0028 & 0.0001 & 0.0005 & 0.0001 & $9.11 * 10^{-5}$ & 0.000149 & 0.0045 \\
\hline 0.9 & 0.0076 & 0.0053 & 0.0001 & 0.0010 & 0.0001 & $9.01 * 10^{-5}$ & 0.0002 & 0.0070 \\
\hline 1 & 0.0120 & 0.0091 & 0.0001 & 0.0010 & $8.82 * 10^{-5}$ & $3.58 * 10^{-5}$ & 0.0002 & 0.0120 \\
\hline 2 & & & 0.0012 & 0.0081 & 0.0004 & 0.0001 & 0.0049 & \\
\hline 3 & & & 0.0077 & 0.0128 & 0.0027 & 0.0007 & 0.0121 & \\
\hline
\end{tabular}

Table 5-6 below shows the mean square error calculated agianst truncated Monte Carlo simulation when $\mathrm{CV}=0.8$ or higher due to frequent negative demands. From Table 5-6, it is clear that when $\mathrm{CV}=0.8$, Hadley's approach, Johnson et al's approach and Johnson et al's modified approach and Zhang and Zhang's approach dominate other formulas in comparison with "truncated" Monte Carlo simulations. Their respective MSE values are $2.18 * 10^{-5}, 2.42 * 10^{-5}, 4.26 * 10^{-5}$, and $1.41 * 10^{-5}$. But when $\mathrm{CV}$ increases to 1 and higher, all MSE of these formulas increases significantly, and thus are not suitable 
for application. Note, however, Zhang and Zhang's approach has a rally when CV=3 against Johnson et al.'s original and modified approaches.

Table 5-7: Mean Square Error versus Monte Carlo simulation (truncated)

\begin{tabular}{|c|c|c|c|c|c|c|c|c|}
\hline MCT & Tradion & Exp & Hadley & Silver & J. et al. & $\begin{array}{l}\text { J. et al. } \\
\bmod \end{array}$ & Z\&Z & Z\&Z app \\
\hline 0.8 & 0.0027 & 0.0014 & $2.18 * 10^{-5}$ & 0.0014 & $2.42 * 10^{-5}$ & $4.26 * 10^{-5}$ & $1.41 * 10^{-5}$ & 0.0027 \\
\hline 0.9 & 0.0050 & 0.0031 & 0.0001 & 0.0024 & 0.0001 & 0.0002 & 0.0001 & 0.0050 \\
\hline 1 & 0.0067 & 0.0042 & 0.0006 & 0.0054 & 0.0006 & 0.0008 & 0.0005 & 0.0067 \\
\hline 2 & & & 0.0058 & 0.0402 & 0.0085 & 0.014 & 0.0027 & \\
\hline 3 & & & 0.0035 & 0.0651 & 0.0098 & 0.0270 & 0.0017 & \\
\hline
\end{tabular}

From Tables 5 and 6 and Figures S-1 to S-10 (see Appendix), as CV increases from $\mathrm{CV}=0.6$ and onwards ( with low $\beta$ service level), the accuracy of the eight formulas is sorted from low to high as follows: exponential approximation, traditional approach, Silver's modified approach, Hadley and Whitin's approach, Zhang and Zhang's approach, Johnson et al.'s approach. Especially, Johnson et al.'s modified approach is significantly better to deal with high values of CV.

\subsubsection{Scaling issues for the general periodic review system}

This subsection focuses on the proper implementation of Silver's modified approach, Johnson et al.'s approach and Johnson et al.'s modified approach when facing the situation where the review interval $R>1$, i.e., a situation described as the "general periodic review." In particular, we implement all three formulas two ways: with and without scaling the relevant inventory system. As discussed in Section 3.7, for example, 
in a general period review system where $L=4, R=2, \mu=200$ and $\sigma=100$, "scaling the system" implies the use of the effective parameters $L^{\prime}=2, R^{\prime}=1, \mu^{\prime}=2 * 200$ and $\sigma^{\prime}=\sqrt{2} * 100$.

Table 5-7 summarizes the results for the three methods (with and without scaling) when compared to the Monte Carlo simulation. This table indicates that one will get an inaccurate $\beta$ service level when adopting Silver's modified approach, Johnson et al.'s approach and Johnson et al.'s modified approach without scaling. For instance, when the base stock level is 1200 units, the simulated system $\beta$ service level is 0.7613 , while silver's modified yields 0.8272 , Johnson et al. gives 0.8125 , and Johnson et al. modified returns 0.8129 . However, when proper scaling is done as illustrated in Table 3-2, silver's modified approach yields 0.7689 , Johnson et al.'s approach gets 0.7600 , and Johnson et al.'s modified approach gives 0.7600 . Similar observations can be made from plots in Figures 6 and 7. Thus, we conclude that scaling is necessary for these three approaches to obtain accurate results.

Table 5-8: Unscaled parameter versus Scaled parameter

\begin{tabular}{|c|c|c|c|c|c|c|c|c|}
\hline S & $\alpha$ & Silver & J. et al. & J. et al. mod & $\mathrm{MC}$ result & $\begin{array}{l}\text { Silver } \\
\text { (unscaled) }\end{array}$ & $\begin{array}{l}\text { J. et al. } \\
\text { (unscaled) }\end{array}$ & $\begin{array}{l}\text { J. et al. mod } \\
\text { (unscaled) }\end{array}$ \\
\hline 1200 & 0.5 & 0.7689 & 0.7600 & 0.7600 & 0.7613 & 0.8272 & 0.8125 & 0.8129 \\
\hline 1206 & 0.51 & 0.7756 & 0.7671 & 0.7671 & 0.7684 & 0.8316 & 0.8172 & 0.8176 \\
\hline 1212 & 0.52 & 0.7821 & 0.7741 & 0.7741 & 0.7755 & 0.8359 & 0.8218 & 0.8222 \\
\hline 1218 & 0.53 & 0.7886 & 0.7809 & 0.7809 & 0.7824 & 0.8402 & 0.8265 & 0.8268 \\
\hline 1225 & 0.54 & 0.7960 & 0.7887 & 0.7888 & 0.7904 & 0.8451 & 0.8318 & 0.8321 \\
\hline
\end{tabular}




\begin{tabular}{|c|c|c|c|c|c|c|c|c|}
\hline 1231 & 0.55 & 0.8022 & 0.7953 & 0.7954 & 0.7971 & 0.8493 & 0.8363 & 0.8366 \\
\hline 1237 & 0.56 & 0.8083 & 0.8018 & 0.8018 & 0.8037 & 0.8534 & 0.8407 & 0.8411 \\
\hline 1243 & 0.57 & 0.8143 & 0.8081 & 0.8081 & 0.8101 & 0.8575 & 0.8451 & 0.8455 \\
\hline 1249 & 0.58 & 0.8201 & 0.8143 & 0.8143 & 0.8163 & 0.8615 & 0.8495 & 0.8498 \\
\hline 1256 & 0.59 & 0.8268 & 0.8213 & 0.8214 & 0.8234 & 0.8661 & 0.8545 & 0.8548 \\
\hline 1262 & 0.6 & 0.8325 & 0.8272 & 0.8273 & 0.8293 & 0.8700 & 0.8587 & 0.8590 \\
\hline 1268 & 0.61 & 0.8380 & 0.8330 & 0.8330 & 0.8351 & 0.8739 & 0.8629 & 0.8632 \\
\hline 1275 & 0.62 & 0.8442 & 0.8396 & 0.8396 & 0.8417 & 0.8783 & 0.8676 & 0.8680 \\
\hline 1281 & 0.63 & 0.8495 & 0.8451 & 0.8451 & 0.8473 & 0.8820 & 0.8716 & 0.8720 \\
\hline 1288 & 0.64 & 0.8554 & 0.8513 & 0.8514 & 0.8535 & 0.8862 & 0.8763 & 0.8766 \\
\hline 1294 & 0.65 & 0.8604 & 0.8566 & 0.8566 & 0.8587 & 0.8898 & 0.8801 & 0.8804 \\
\hline 1301 & 0.66 & 0.8661 & 0.8625 & 0.8625 & 0.8646 & 0.8938 & 0.8846 & 0.8849 \\
\hline 1308 & 0.67 & 0.8716 & 0.8682 & 0.8683 & 0.8703 & 0.8978 & 0.8889 & 0.8892 \\
\hline 1315 & 0.68 & 0.8770 & 0.8738 & 0.8739 & 0.8759 & 0.9017 & 0.8932 & 0.8934 \\
\hline 1321 & 0.69 & 0.8814 & 0.8785 & 0.8785 & 0.8806 & 0.9050 & 0.8967 & 0.8970 \\
\hline 1328 & 0.7 & 0.8865 & 0.8838 & 0.8838 & 0.8859 & 0.9087 & 0.9008 & 0.9010 \\
\hline 1336 & 0.71 & 0.8921 & 0.8896 & 0.8896 & 0.8918 & 0.9129 & 0.9053 & 0.9056 \\
\hline 1343 & 0.72 & 0.8968 & 0.8945 & 0.8945 & 0.8967 & 0.9164 & 0.9091 & 0.9094 \\
\hline 1350 & 0.73 & 0.9014 & 0.8993 & 0.8993 & 0.9015 & 0.9198 & 0.9129 & 0.9131 \\
\hline 1358 & 0.74 & 0.9065 & 0.9045 & 0.9045 & 0.9067 & 0.9236 & 0.9171 & 0.9173 \\
\hline 1365 & 0.75 & 0.9108 & 0.9089 & 0.9089 & 0.9111 & 0.9269 & 0.9206 & 0.9208 \\
\hline 1373 & 0.76 & 0.9155 & 0.9138 & 0.9138 & 0.9160 & 0.9305 & 0.9245 & 0.9247 \\
\hline
\end{tabular}




\begin{tabular}{|c|c|c|c|c|c|c|c|c|}
\hline 1381 & 0.77 & 0.9200 & 0.9184 & 0.9184 & 0.9207 & 0.9339 & 0.9283 & 0.9285 \\
\hline 1389 & 0.78 & 0.9243 & 0.9229 & 0.9229 & 0.9253 & 0.9373 & 0.9319 & 0.9321 \\
\hline 1398 & 0.79 & 0.9290 & 0.9277 & 0.9277 & 0.9302 & 0.9409 & 0.9359 & 0.9361 \\
\hline 1406 & 0.8 & 0.9329 & 0.9318 & 0.9318 & 0.9344 & 0.9440 & 0.9392 & 0.9394 \\
\hline 1415 & 0.81 & 0.9372 & 0.9361 & 0.9361 & 0.9388 & 0.9473 & 0.9429 & 0.9431 \\
\hline 1424 & 0.82 & 0.9412 & 0.9403 & 0.9403 & 0.9430 & 0.9505 & 0.9464 & 0.9465 \\
\hline 1434 & 0.83 & 0.9454 & 0.9446 & 0.9446 & 0.9474 & 0.9539 & 0.9501 & 0.9502 \\
\hline 1444 & 0.84 & 0.9494 & 0.9487 & 0.9487 & 0.9515 & 0.9571 & 0.9535 & 0.9537 \\
\hline 1454 & 0.85 & 0.9532 & 0.9526 & 0.9526 & 0.9553 & 0.9601 & 0.9569 & 0.9570 \\
\hline 1465 & 0.86 & 0.9571 & 0.9565 & 0.9565 & 0.9594 & 0.9632 & 0.9603 & 0.9604 \\
\hline 1476 & 0.87 & 0.9607 & 0.9602 & 0.9602 & 0.9631 & 0.9662 & 0.9635 & 0.9636 \\
\hline 1488 & 0.88 & 0.9644 & 0.9640 & 0.9640 & 0.9668 & 0.9692 & 0.9668 & 0.9669 \\
\hline 1500 & 0.89 & 0.9678 & 0.9674 & 0.9674 & 0.9701 & 0.9720 & 0.9698 & 0.9699 \\
\hline 1514 & 0.9 & 0.9714 & 0.9711 & 0.9711 & 0.9735 & 0.9750 & 0.9731 & 0.9732 \\
\hline 1528 & 0.91 & 0.9746 & 0.9744 & 0.9744 & 0.9766 & 0.9777 & 0.9761 & 0.9762 \\
\hline 1544 & 0.92 & 0.9780 & 0.9778 & 0.9778 & 0.9798 & 0.9806 & 0.9791 & 0.9792 \\
\hline 1561 & 0.93 & 0.9811 & 0.9810 & 0.9810 & 0.9829 & 0.9833 & 0.9820 & 0.9821 \\
\hline 1581 & 0.94 & 0.9843 & 0.9842 & 0.9842 & 0.9860 & 0.9860 & 0.9850 & 0.9851 \\
\hline 1603 & 0.95 & 0.9873 & 0.9872 & 0.9872 & 0.9890 & 0.9886 & 0.9878 & 0.9879 \\
\hline 1629 & 0.96 & 0.9902 & 0.9901 & 0.9901 & 0.9918 & 0.9911 & 0.9905 & 0.9906 \\
\hline 1661 & 0.97 & 0.9929 & 0.9929 & 0.9929 & 0.9944 & 0.9936 & 0.9932 & 0.9932 \\
\hline 1703 & 0.98 & 0.9955 & 0.9955 & 0.9955 & 0.9967 & 0.9955 & 0.9955 & 0.9955 \\
\hline
\end{tabular}




\begin{tabular}{lllllllll}
\hline 1770 & 0.99 & 0.9979 & 0.9979 & 0.9979 & 0.9990 & 0.9979 & 0.9979 & 0.9979 \\
\hline
\end{tabular}

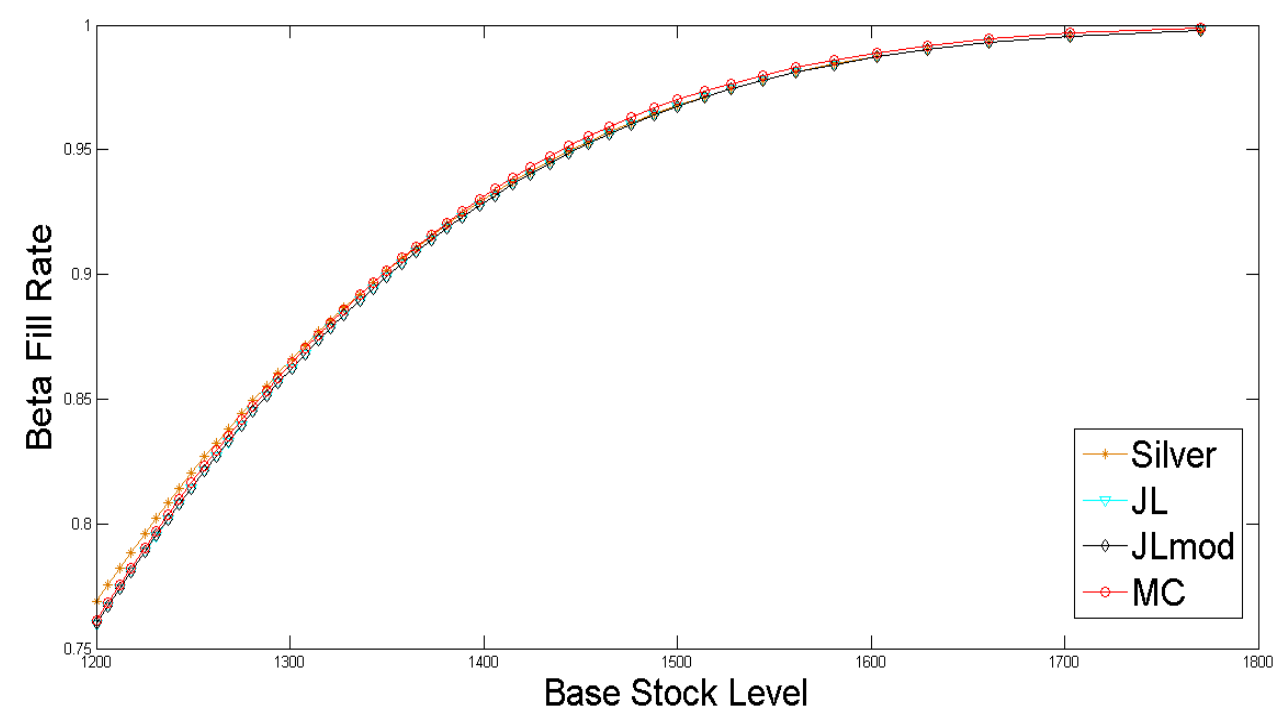

Figure 5-6: Modified Silver and Johnson et al.'s with Scaling

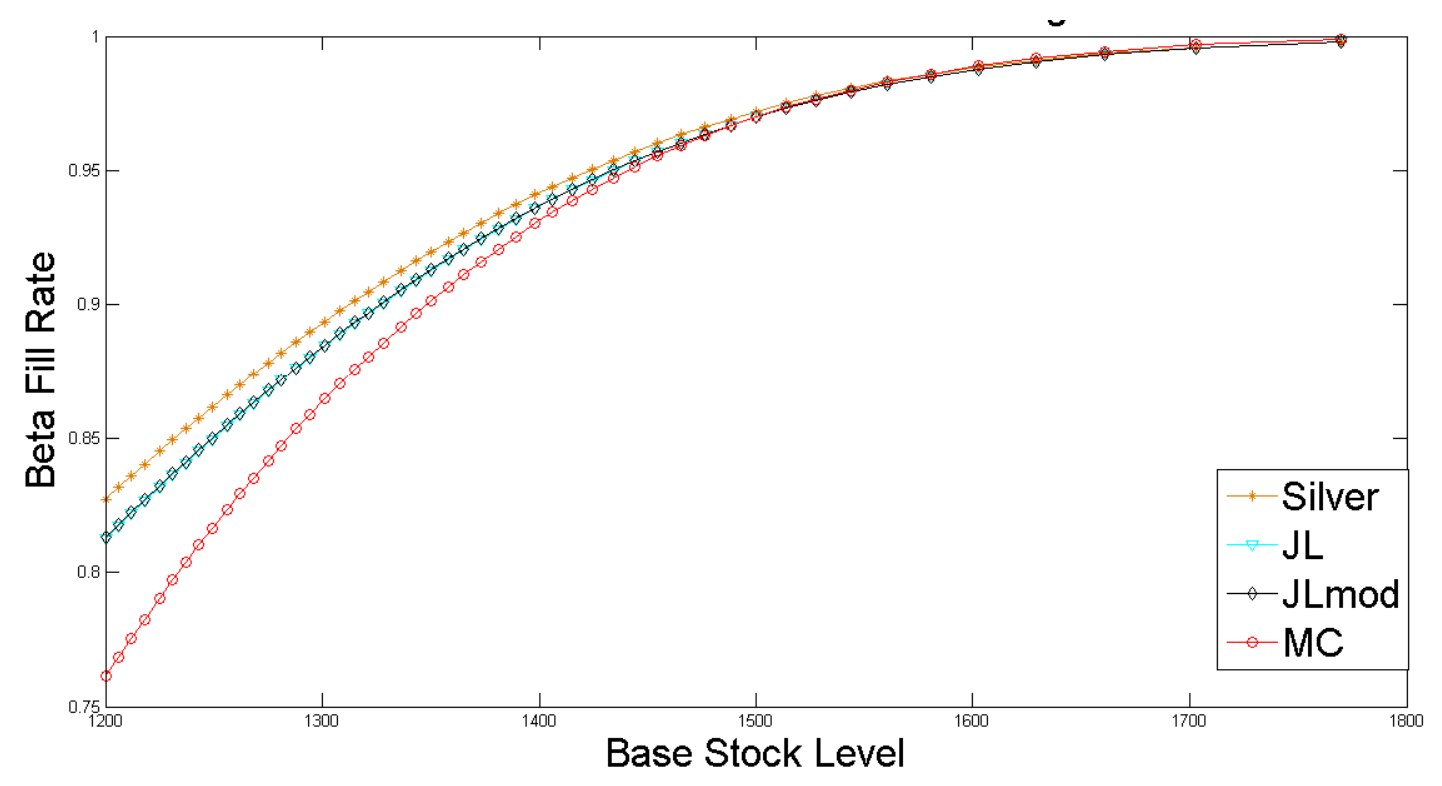

Figure 5-7: Modified Silver and Johnson et al.'s without Scaling 


\section{CHAPTER 6 CONCLUSIONS AND FUTURE RESEARCH}

\subsection{Conclusions}

In this thesis, we study the $\beta$ service level as a measure for a supply chain's ability to meet customers' demands immediately by its on-hand inventory. Among three service levels, $\alpha, \beta$ and $\gamma$ service levels, the system $\beta$ service level is most commonly used in practice in the supply chains and logistics industry. The focus of this thesis is on the single-stage periodic review inventory system. Through the Monte Carlo simulation implemented in MATLAB, we investigate the behavior of the $\beta$ service level under various scenarios as well as compare the numerical performance of the eight existing fill rate expressions in the literature.

In our first set of experiments, we model a general periodic review system in order to observe the behavior of $\beta$ service level under different scenarios. From a base setting of the underlying inventory system, we vary one parameter at a time, which includes: the base stock level, the review interval, the replenishment lead time and the coefficient of variation for the random demand. The observations are summarized as follows.

Firstly, as the base stock level increase, the $\beta$ service level increases. Secondly, the $\alpha$ service level changes slightly more rapidly than the $\beta$ service level given the same increment of base stock level, which indicates that the $\alpha$ service level is more sensitive to 
the change of base stock level. Third, as the coefficient of variation for the random periodic demand increases, the $\beta$ service level decreases. Lastly, both the replenishment lead time and review interval have negative effect on the system's $\beta$ service level, with the replenishment lead time imposing larger effect than the review interval.

In the second set of our experiments, the goal is to numerically evaluate the accuracy of the eight fill rate expressions, benchmarking against either the Monte Carlo simulation or "truncated" Monte Carlo simulation (when appropriate). The research questions center around: which formula(s) perform the best under certain conditions? We give considerations for an inventory system with: high or low fill rate, high or low demand variability and unit or general period review. Several observations are made and they are summarized below.

First, all formulas perform well when the $\beta$ service level is as high as $70 \%$. However, when the $\beta$ service level falls below $60 \%$ or lower, Zhang and Zhang, Hadley and Whitin, Johnson et al.'s and Jonson et al.'s modified approaches produce accurate $\beta$ service level, whereas Silver's modified, traditional, exponential approximation and Zhang and Zhang's approximation do not.

Second, when the CV for random demand is 0.5 or lower, all formulas perform well. However, when $\mathrm{CV}$ increases to 0.6 or higher, the accuracy of various formulas is sorted from low to high as follows: Exponential approximation, traditional approach, Silver's modified, Hadley and Whitin's, Zhang and Zhang's, Johnson et al.'s, and Johnson et al.'s modified approach. It is worth noting that when CV becomes 2 or higher, the traditional, exponential approximation and Zhang and Zhang's approximation all 
experience negative fill rate occasionally. Thus, they should not be considered for use if $\mathrm{CV}$ is as high as 2. Finally, Johnson et al.'s modified approach is significantly better than others for dealing with high values of $\mathrm{CV}$.

In addition, we also use the Monte Carlo simulation to study the fill rate for the so-called "general periodic review system." Our numerical results indicate that scaling is necessary for Silver's modified, Johnson et al.'s and Johnson et al.'s modified approaches. On the other hand, traditional approach, exponential approximation and Hadley and Whitin's approach require very minimum treatment in order to handle the general periodic review system. Finally, Zhang and Zhang's approach is designed to handle the general system directly and thus is the most versatile.

\subsection{Future Research}

There are several directions for future research. First, while the current thesis demonstrates the accuracy of existing fill rate formulas are mostly of high quality, little research is done for expressing the system fill rate for multi-stage inventory systems. Sobel (2004) and Zhang et al. (2010) are among the few publications in this endeavor, we plan to study this topic in the near future.

Second, while the current thesis demonstrates that a "general period review system" can be treated as a "unit periodic review system" for a single-stage inventory system by simple scaling technique, it remains open whether such technique would work

for multi-stage inventory system. For example, when the review intervals of upstream and downstream supply chains do not match, the choice of a proper "scaling factor" may 
be challenging. In other words, the legitimacy of the notion of "general periodic review" is still an open question. We would like to at least investigate it numerically.

Third, even if "scaling" a multi-stage inventory system is feasible, the implication of holding cost may be interesting to examine as well.

Last but not least, it would be an interesting topic to develop efficient numerical algorithms for supply chain optimization problems with service level constraints. Due to the underlying complexity involving the evaluation of fill rate in the constraints, one expect heuristics such as genetic algorithm and simulated annealing to be suitable for its solution. 


\section{REFERENCES}

Anderson, H.L. (1986). Metropolis, Monte Carlo and the MANIAC. Los Alamos Science 14, 96-108.

Bashyam, S. \& Fu, M.C. (1998). Optimization of (s, S) inventory systems with random lead times and a service level constraint. Management Science 44(12), 243-256.

Beamon, B.M. (1999). Measuring supply chain performance. International Journal of Operations \& Production Management 19(3), 275-292.

Char, B. W. (1988). MAPLE reference manual. Watcom Publications.

Chase, R.B. \& Aquilano, N.J. (1992). Production and operations management. Homewood, IL: Richard D. Irwin Inc.

Hadley, G.J. \& Whitin, T.M. (1963). Analysis of inventory systems. Englewood Cliffs, NJ: Prentice-Hall.

Johnson, M.E., Lee, H.L., Davis, T. \& Hall R. (1995). Expressions for item fill rates in periodic inventory systems. Naval Research Logistics 42, 57-80.

MATLAB User's Manual, M. U. S. (2002). The Mathworks Inc. Natick, Massachusetts.

Nahmias, S. (1989). Production and Operations Management. Homewood, IL: Richard D. Irwin Inc. 
Ouyang, L. \& Wu, K. (1997). Mixture inventory model involving variable lead time with a service level constraint. Computers Ops Res 24(9), 875-882.

Sawilowsky, S.S. \& Fahoome, G.C. (2003). Statistics via monte carlo simulation with fortran. Rochester Hills, MI: JMASM.

Silver, E.A. (1970). A modified formula for calculating customer service under continuous inventory review. AIIE Transactions 2(3), 241-245.

Silver, E.A. \& Bischak, D.P. (2011). The exact fill rate in a periodic review stoch system under normally distributed demand. International Journal of Management Science 39, 346-349.

Silver, E.A. \& Costa, D. (1995). Service and inventory level implications of a particular simple method of establishing the reorder point. Information Systems \& Operational Research 33(2), 133-144.

Sobel, M.J. (2004). Fill rates of single-stage and multistage supply systems. Manufacturing \& Service Operations Management 6(1), 41-52.

Tempelmeier, H. (2000). Inventory service-levels in the customer supply chain. OR Spektrum 22, 361-380.

Thomas, D.J. (2005). Measuring item fill-rate performance in a finite horizon. Manufacturing \& Service Operations Management 7(1), 74-80.

Vollmann, T. E., Berry, W. L. \& Whybark, D. C. (1988). Manufacturing planning and control systems. Homewood, IL: Richard D. Irwin Inc. 
Zhang, J., Bai, L. \& He, Y. (2010) Fill rate of general periodic review two-stage inventory systems. International Journal of Operational Research 8(1), 62-84.

Zhang, J. \& Zhang, J. (2007). Fill rate of single-stage general periodic review inventory systems. Operation Research Letters 35, 503-509. 


\section{APPENDIX}

Table S-1: MC Results versus Computation Results When CV=0.1

\begin{tabular}{|c|c|c|c|c|c|c|c|c|c|c|c|c|}
\hline $\mathrm{CV}$ & $\alpha$ & $\mathrm{S}$ & $\mathrm{MC}$ & Tradition & EXP & Hadley & Silver & J. et al. & $\begin{array}{l}\text { J. et al. } \\
\text { mod }\end{array}$ & Z\&Z & Z\&Z app & Trunc. MC \\
\hline 0.1 & 0.3 & 1174 & 0.9117 & 0.9119 & 0.9173 & 0.9119 & 0.9119 & 0.9119 & 0.9119 & 0.9119 & 0.9119 & 0.9117 \\
\hline 0.1 & 0.4 & 1188 & 0.9345 & 0.9347 & 0.9361 & 0.9347 & 0.9347 & 0.9347 & 0.9347 & 0.9347 & 0.9347 & 0.9345 \\
\hline 0.1 & 0.5 & 1200 & 0.9510 & 0.9511 & 0.9512 & 0.9511 & 0.9511 & 0.9511 & 0.9511 & 0.9511 & 0.9511 & 0.9510 \\
\hline 0.1 & 0.6 & 1212 & 0.9648 & 0.9647 & 0.9643 & 0.9647 & 0.9647 & 0.9647 & 0.9647 & 0.9647 & 0.9647 & 0.9648 \\
\hline 0.1 & 0.7 & 1226 & 0.9772 & 0.9769 & 0.9766 & 0.9769 & 0.9769 & 0.9769 & 0.9769 & 0.9769 & 0.9769 & 0.9772 \\
\hline 0.1 & 0.8 & 1241 & 0.9869 & 0.9862 & 0.9861 & 0.9862 & 0.9862 & 0.9862 & 0.9862 & 0.9862 & 0.9862 & 0.9869 \\
\hline 0.1 & 0.9 & 1263 & 0.9949 & 0.9943 & 0.9943 & 0.9943 & 0.9943 & 0.9943 & 0.9943 & 0.9943 & 0.9943 & 0.9949 \\
\hline 0.1 & 0.99 & 1314 & 0.9995 & 0.9996 & 0.9996 & 0.9996 & 0.9996 & 0.9996 & 0.9996 & 0.9996 & 0.9996 & 0.9995 \\
\hline
\end{tabular}

Table S-2: MC Results versus Computation Results When CV=0.2

\begin{tabular}{|c|c|c|c|c|c|c|c|c|c|c|c|c|}
\hline $\mathrm{CV}$ & $\alpha$ & $\mathrm{S}$ & $\mathrm{MC}$ & Tradition & EXP & Hadley & Silver & J. et al. & $\begin{array}{l}\text { J. et al. } \\
\text { mod }\end{array}$ & Z\&Z & Z\&Z app & Trunc. MC \\
\hline 0.2 & 0.3 & 1149 & 0.8365 & 0.8256 & 0.8360 & 0.8256 & 0.8256 & 0.8256 & 0.8256 & 0.8256 & 0.8256 & 0.8365 \\
\hline 0.2 & 0.4 & 1175 & 0.8783 & 0.8679 & 0.8709 & 0.8679 & 0.8679 & 0.8679 & 0.8679 & 0.8679 & 0.8679 & 0.8783 \\
\hline 0.2 & 0.5 & 1200 & 0.9113 & 0.9023 & 0.9024 & 0.9023 & 0.9023 & 0.9023 & 0.9023 & 0.9023 & 0.9023 & 0.9113 \\
\hline 0.2 & 0.6 & 1225 & 0.9375 & 0.9304 & 0.9297 & 0.9304 & 0.9304 & 0.9304 & 0.9304 & 0.9304 & 0.9304 & 0.9375 \\
\hline
\end{tabular}




\begin{tabular}{|c|c|c|c|c|c|c|c|c|c|c|c|c|}
\hline 0.2 & 0.7 & 1251 & 0.9587 & 0.9531 & 0.9525 & 0.9531 & 0.9531 & 0.9531 & 0.9531 & 0.9531 & 0.9531 & 0.9587 \\
\hline 0.2 & 0.8 & 1282 & 0.9760 & 0.9724 & 0.9722 & 0.9724 & 0.9724 & 0.9724 & 0.9724 & 0.9724 & 0.9724 & 0.9760 \\
\hline 0.2 & 0.9 & 1326 & 0.9902 & 0.9885 & 0.9885 & 0.9885 & 0.9885 & 0.9885 & 0.9885 & 0.9885 & 0.9885 & 0.9902 \\
\hline 0.2 & 0.99 & 1428 & 0.9993 & 0.9992 & 0.9992 & 0.9992 & 0.9992 & 0.9992 & 0.9992 & 0.9992 & 0.9992 & 0.9993 \\
\hline
\end{tabular}

Table S-3: MC Results versus Computation Results When CV=0.3

\begin{tabular}{|c|c|c|c|c|c|c|c|c|c|c|c|c|}
\hline $\mathrm{CV}$ & $\alpha$ & $\mathrm{S}$ & $\mathrm{MC}$ & Tradition & EXP & Hadley & Silver & J. et al. & $\begin{array}{l}\text { J. et al. } \\
\text { mod }\end{array}$ & Z\&Z & Z\&Z app & $\begin{array}{l}\text { Trunc. } \\
\text { MC }\end{array}$ \\
\hline 0.3 & 0.3 & 1123 & 0.7441 & 0.7375 & 0.7533 & 0.7378 & 0.7393 & 0.7378 & 0.7378 & 0.7378 & 0.7375 & 0.7441 \\
\hline 0.3 & 0.4 & 1163 & 0.8067 & 0.8026 & 0.8070 & 0.8027 & 0.8034 & 0.8027 & 0.8027 & 0.8027 & 0.8026 & 0.8067 \\
\hline 0.3 & 0.5 & 1200 & 0.8557 & 0.8534 & 0.8536 & 0.8535 & 0.8538 & 0.8535 & 0.8535 & 0.8535 & 0.8534 & 0.8557 \\
\hline 0.3 & 0.6 & 1237 & 0.8952 & 0.8951 & 0.8940 & 0.8951 & 0.8952 & 0.8951 & 0.8951 & 0.8951 & 0.8951 & 0.8952 \\
\hline 0.3 & 0.7 & 1277 & 0.9283 & 0.9300 & 0.9291 & 0.9300 & 0.9301 & 0.9300 & 0.9300 & 0.9300 & 0.9300 & 0.9283 \\
\hline 0.3 & 0.8 & 1324 & 0.9571 & 0.9592 & 0.9588 & 0.9592 & 0.9592 & 0.9592 & 0.9592 & 0.9591 & 0.9592 & 0.9571 \\
\hline 0.3 & 0.9 & 1388 & 0.9826 & 0.9825 & 0.9826 & 0.9825 & 0.9825 & 0.9825 & 0.9825 & 0.9825 & 0.9825 & 0.9826 \\
\hline 0.3 & 0.99 & 1542 & 0.9993 & 0.9988 & 0.9988 & 0.9988 & 0.9988 & 0.9988 & 0.9988 & 0.9994 & 0.9988 & 0.9993 \\
\hline
\end{tabular}

Table S-4: MC Results versus Computation Results When CV=0.4 


\begin{tabular}{|c|c|c|c|c|c|c|c|c|c|c|c|c|}
\hline $\mathrm{CV}$ & $\alpha$ & $\mathrm{S}$ & $\mathrm{MC}$ & Tradition & EXP & Hadley & Silver & J. et al. & $\begin{array}{l}\text { J. et al. } \\
\text { mod }\end{array}$ & Z\&Z & Z\&Z app & $\begin{array}{l}\text { Trunc. } \\
\text { MC }\end{array}$ \\
\hline 0.4 & 0.3 & 1097 & 0.6547 & 0.6494 & 0.6706 & 0.6544 & 0.6633 & 0.6544 & 0.6544 & 0.6544 & 0.6494 & 0.6547 \\
\hline 0.4 & 0.4 & 1150 & 0.7380 & 0.7358 & 0.7418 & 0.7378 & 0.7430 & 0.7378 & 0.7378 & 0.7378 & 0.7358 & 0.7380 \\
\hline 0.4 & 0.5 & 1200 & 0.8042 & 0.8046 & 0.8048 & 0.8054 & 0.8083 & 0.8054 & 0.8054 & 0.8054 & 0.8046 & 0.8042 \\
\hline 0.4 & 0.6 & 1250 & 0.8597 & 0.8608 & 0.8594 & 0.8611 & 0.8626 & 0.8611 & 0.8611 & 0.8610 & 0.8608 & 0.8597 \\
\hline 0.4 & 0.7 & 1303 & 0.9063 & 0.9069 & 0.9057 & 0.9070 & 0.9077 & 0.9070 & 0.9070 & 0.9070 & 0.9069 & 0.9063 \\
\hline 0.4 & 0.8 & 1365 & 0.9451 & 0.9454 & 0.9449 & 0.9454 & 0.9456 & 0.9454 & 0.9454 & 0.9454 & 0.9454 & 0.9451 \\
\hline 0.4 & 0.9 & 1451 & 0.9756 & 0.9768 & 0.9768 & 0.9768 & 0.9768 & 0.9768 & 0.9768 & 0.9768 & 0.9768 & 0.9756 \\
\hline 0.4 & 0.99 & 1656 & 0.9973 & 0.9983 & 0.9984 & 0.9983 & 0.9983 & 0.9983 & 0.9983 & 0.9983 & 0.9983 & 0.9973 \\
\hline
\end{tabular}

Table S-5: MC Results versus Computation Results When CV=0.5

\begin{tabular}{|c|c|c|c|c|c|c|c|c|c|c|c|c|}
\hline $\mathrm{CV}$ & $\alpha$ & $\mathrm{S}$ & $\mathrm{MC}$ & Tradition & EXP & Hadley & Silver & J. et al. & $\begin{array}{l}\text { J. et al. } \\
\text { mod }\end{array}$ & Z\&Z & Z\&Z app & $\begin{array}{l}\text { Trunc. } \\
\text { MC }\end{array}$ \\
\hline 0.5 & 0.3 & 1072 & 0.5830 & 0.5631 & 0.5892 & 0.5831 & 0.6043 & 0.5831 & 0.5832 & 0.5831 & 0.5631 & 0.5830 \\
\hline 0.5 & 0.4 & 1138 & 0.6810 & 0.6705 & 0.6779 & 0.6798 & 0.6939 & 0.6798 & 0.6799 & 0.6798 & 0.6705 & 0.6810 \\
\hline 0.5 & 0.5 & 1200 & 0.7613 & 0.7557 & 0.7560 & 0.7600 & 0.7689 & 0.7600 & 0.7600 & 0.7599 & 0.7557 & 0.7613 \\
\hline 0.5 & 0.6 & 1262 & 0.8293 & 0.8255 & 0.8237 & 0.8272 & 0.8325 & 0.8272 & 0.8273 & 0.8272 & 0.8255 & 0.8293 \\
\hline 0.5 & 0.7 & 1328 & 0.8859 & 0.8831 & 0.8816 & 0.8838 & 0.8865 & 0.8838 & 0.8838 & 0.8837 & 0.8831 & 0.8859 \\
\hline 0.5 & 0.8 & 1406 & 0.9344 & 0.9316 & 0.9310 & 0.9318 & 0.9329 & 0.9318 & 0.9318 & 0.9317 & 0.9316 & 0.9344 \\
\hline
\end{tabular}




\begin{tabular}{llllllllllllllll}
\hline 0.5 & 0.9 & 1514 & 0.9735 & 0.9710 & 0.9711 & 0.9711 & 0.9711 & 0.9711 & 0.9711 & 0.9710 & 0.9710 & 0.9735 \\
0.5 & 0.99 & 1770 & 0.9990 & 0.9979 & 0.9979 & 0.9979 & 0.9979 & 0.9979 & 0.9979 & 0.9979 & 0.9979 & 0.9990 \\
\hline
\end{tabular}

Table S-6: MC Results versus Computation Results When CV=0.6

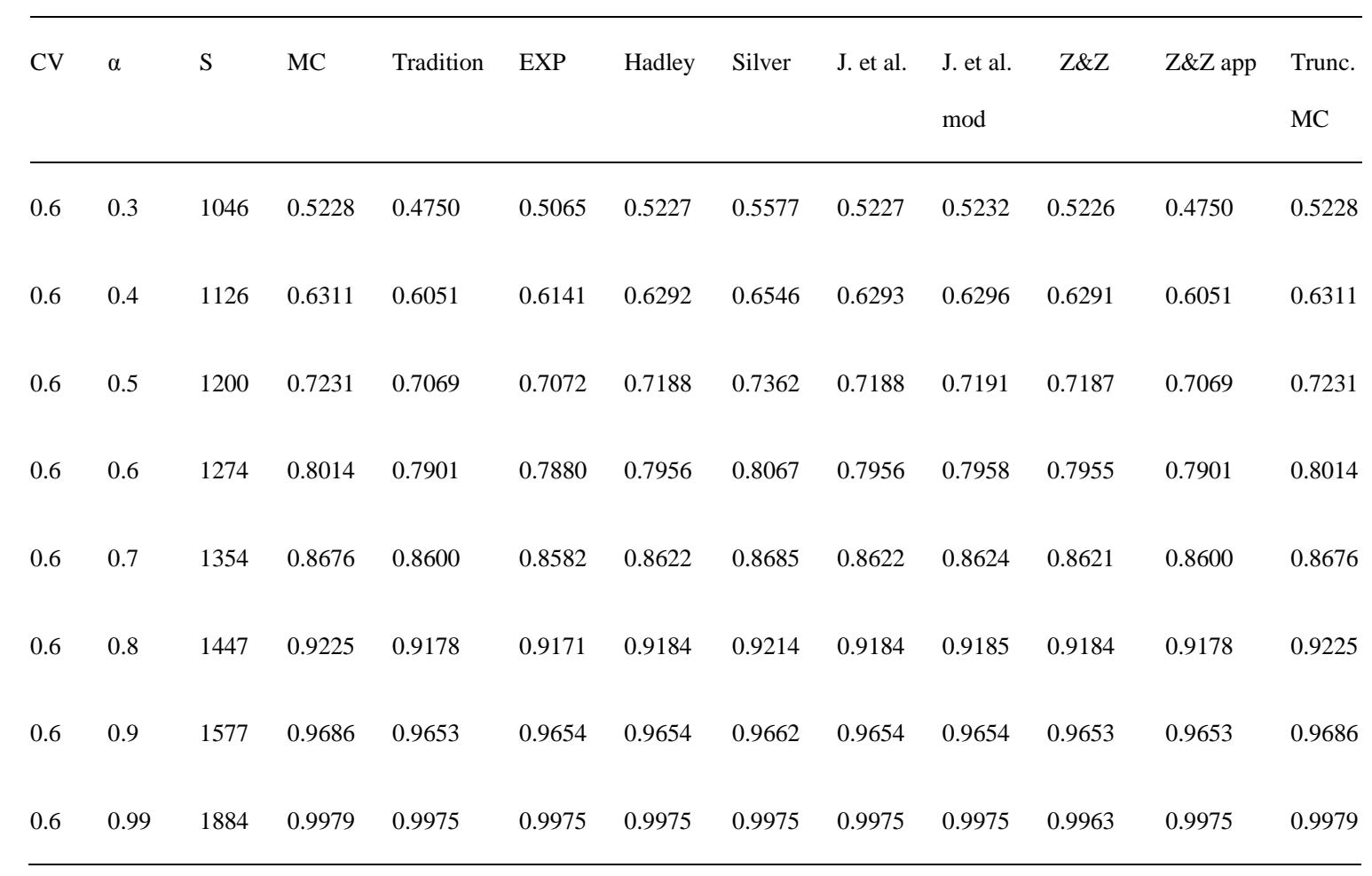

Table S-7: MC Results versus Computation Results When CV=0.7

\begin{tabular}{|c|c|c|c|c|c|c|c|c|c|c|c|c|}
\hline $\mathrm{CV}$ & $\alpha$ & S & MC & Tradition & EXP & Hadley & Silver & J. et al. & $\begin{array}{l}\text { J. et al. } \\
\text { mod }\end{array}$ & Z\&Z & Z\&Z app & $\begin{array}{l}\text { Trunc. } \\
\text { MC }\end{array}$ \\
\hline 0.7 & 0.3 & 1020 & 0.4742 & 0.3870 & 0.4238 & 0.4732 & 0.5220 & 0.4734 & 0.4747 & 0.4728 & 0.3870 & 0.4742 \\
\hline
\end{tabular}




\begin{tabular}{|c|c|c|c|c|c|c|c|c|c|c|c|c|}
\hline 0.7 & 0.4 & 1113 & 0.5854 & 0.5383 & 0.5489 & 0.5847 & 0.6224 & 0.5848 & 0.5859 & 0.5843 & 0.5383 & 0.5854 \\
\hline 0.7 & 0.5 & 1200 & 0.6792 & 0.6580 & 0.6584 & 0.6821 & 0.7095 & 0.6822 & 0.6832 & 0.6817 & 0.6580 & 0.6792 \\
\hline 0.7 & 0.6 & 1287 & 0.7614 & 0.7558 & 0.7534 & 0.7674 & 0.7859 & 0.7675 & 0.7683 & 0.7670 & 0.7558 & 0.7614 \\
\hline 0.7 & 0.7 & 1380 & 0.8346 & 0.8370 & 0.8348 & 0.8419 & 0.8530 & 0.8419 & 0.8424 & 0.8414 & 0.8370 & 0.8346 \\
\hline 0.7 & 0.8 & 1489 & 0.8997 & 0.9045 & 0.9037 & 0.9061 & 0.9116 & 0.9061 & 0.9065 & 0.9057 & 0.9045 & 0.8997 \\
\hline 0.7 & 0.9 & 1639 & 0.9558 & 0.9593 & 0.9594 & 0.9596 & 0.9613 & 0.9596 & 0.9597 & 0.9592 & 0.9593 & 0.9558 \\
\hline 0.7 & 0.99 & 1998 & 0.9968 & 0.9971 & 0.9971 & 0.9971 & 0.9972 & 0.9971 & 0.9971 & 0.9967 & 0.9971 & 0.9968 \\
\hline
\end{tabular}

Table S-8: MC Results versus Computation Results When CV=0.8

\begin{tabular}{|c|c|c|c|c|c|c|c|c|c|c|c|c|}
\hline $\mathrm{CV}$ & $\alpha$ & S & MC & Tradition & EXP & Hadley & Silver & J. et al. & $\begin{array}{l}\text { J. et al. } \\
\text { mod }\end{array}$ & $\mathrm{Z} \& \mathrm{Z}$ & Z\&Z app & $\begin{array}{l}\text { Trunc. } \\
\text { MC }\end{array}$ \\
\hline 0.8 & 0.3 & 994 & 0.4549 & 0.2989 & 0.3411 & 0.4324 & 0.4941 & 0.4330 & 0.4356 & 0.4311 & 0.2989 & 0.4244 \\
\hline 0.8 & 0.4 & 1101 & 0.5646 & 0.4730 & 0.4850 & 0.5475 & 0.5975 & 0.5480 & 0.5504 & 0.5462 & 0.4730 & 0.5416 \\
\hline 0.8 & 0.5 & 1200 & 0.6626 & 0.6092 & 0.6096 & 0.6496 & 0.6876 & 0.6499 & 0.6520 & 0.6483 & 0.6092 & 0.6466 \\
\hline 0.8 & 0.6 & 1299 & 0.7477 & 0.7205 & 0.7177 & 0.7410 & 0.7677 & 0.7411 & 0.7429 & 0.7396 & 0.7205 & 0.7385 \\
\hline 0.8 & 0.7 & 1406 & 0.8221 & 0.8139 & 0.8115 & 0.8229 & 0.8397 & 0.8230 & 0.8242 & 0.8215 & 0.8139 & 0.8174 \\
\hline 0.8 & 0.8 & 1530 & 0.8912 & 0.8907 & 0.8898 & 0.8938 & 0.9027 & 0.8939 & 0.8947 & 0.8925 & 0.8907 & 0.8884 \\
\hline 0.8 & 0.9 & 1702 & 0.9559 & 0.9536 & 0.9537 & 0.9541 & 0.9571 & 0.9542 & 0.9545 & 0.9528 & 0.9536 & 0.9544 \\
\hline 0.8 & 0.99 & 2112 & 0.9986 & 0.9967 & 0.9967 & 0.9967 & 0.9968 & 0.9967 & 0.9967 & 0.9954 & 0.9967 & 0.9981 \\
\hline
\end{tabular}


Table S-9: MC Results versus Computation Results When CV=0.9

\begin{tabular}{|c|c|c|c|c|c|c|c|c|c|c|c|c|}
\hline $\mathrm{CV}$ & $\alpha$ & $\mathrm{S}$ & $\mathrm{MC}$ & Tradition & EXP & Hadley & Silver & J. et al. & $\begin{array}{l}\text { J. et al. } \\
\text { mod }\end{array}$ & $Z \& Z$ & Z\&Z app & $\begin{array}{l}\text { Trunc. } \\
\text { MC }\end{array}$ \\
\hline 0.9 & 0.3 & 969 & 0.4049 & 0.2126 & 0.2598 & 0.3992 & 0.4729 & 0.4005 & 0.4048 & 0.3961 & 0.2126 & 0.3728 \\
\hline 0.9 & 0.4 & 1088 & 0.5266 & 0.4062 & 0.4198 & 0.5144 & 0.5765 & 0.5153 & 0.5196 & 0.5113 & 0.4062 & 0.5011 \\
\hline 0.9 & 0.5 & 1200 & 0.6371 & 0.5603 & 0.5608 & 0.6207 & 0.6695 & 0.6214 & 0.6252 & 0.6176 & 0.5603 & 0.6179 \\
\hline 0.9 & 0.6 & 1312 & 0.7361 & 0.6862 & 0.6830 & 0.7176 & 0.7531 & 0.7181 & 0.7212 & 0.7145 & 0.6862 & 0.7224 \\
\hline 0.9 & 0.7 & 1431 & 0.8249 & 0.7901 & 0.7873 & 0.8045 & 0.8278 & 0.8047 & 0.8072 & 0.8014 & 0.7901 & 0.8157 \\
\hline 0.9 & 0.8 & 1571 & 0.8848 & 0.8770 & 0.8759 & 0.8821 & 0.8948 & 0.8822 & 0.8838 & 0.8791 & 0.8770 & 0.8955 \\
\hline 0.9 & 0.9 & 1765 & 0.9510 & 0.9478 & 0.9480 & 0.9488 & 0.9534 & 0.9489 & 0.9496 & 0.9436 & 0.9478 & 0.9566 \\
\hline 0.9 & 0.99 & 2226 & 0.9960 & 0.9963 & 0.9963 & 0.9963 & 0.9965 & 0.9963 & 0.9963 & 0.9932 & 0.9963 & 0.9959 \\
\hline
\end{tabular}

Table S-10: MC Results versus Computation Results When CV=1

\begin{tabular}{|c|c|c|c|c|c|c|c|c|c|c|c|c|}
\hline $\mathrm{CV}$ & $\alpha$ & S & $\mathrm{MC}$ & Tradition & EXP & Hadley & Silver & J. et al. & $\begin{array}{l}\text { J. et al. } \\
\bmod \end{array}$ & Z\&Z & Z\&Z app & $\begin{array}{l}\text { Trunc. } \\
\text { MC }\end{array}$ \\
\hline 1 & 0.3 & 943 & 0.3748 & 0.1245 & 0.1771 & 0.3698 & 0.4549 & 0.3722 & 0.3747 & 0.3642 & 0.1245 & 0.3181 \\
\hline 1 & 0.4 & 1076 & 0.4932 & 0.3409 & 0.3559 & 0.4862 & 0.5600 & 0.4880 & 0.4945 & 0.4805 & 0.3409 & 0.4458 \\
\hline 1 & 0.5 & 1200 & 0.6092 & 0.5115 & 0.5120 & 0.5948 & 0.6544 & 0.5961 & 0.6021 & 0.5891 & 0.5115 & 0.5691 \\
\hline 1 & 0.6 & 1324 & 0.7118 & 0.6509 & 0.6474 & 0.6954 & 0.7401 & 0.6963 & 0.7014 & 0.6898 & 0.6509 & 0.6844 \\
\hline 1 & 0.7 & 1457 & 0.8005 & 0.7670 & 0.7639 & 0.7880 & 0.8180 & 0.7885 & 0.7924 & 0.7823 & 0.7670 & 0.7857 \\
\hline
\end{tabular}




\begin{tabular}{|c|c|c|c|c|c|c|c|c|c|c|c|c|}
\hline 1 & 0.8 & 1612 & 0.8799 & 0.8632 & 0.8619 & 0.8710 & 0.8880 & 0.8712 & 0.8739 & 0.8653 & 0.8632 & 0.8737 \\
\hline 1 & 0.9 & 1828 & 0.9497 & 0.9421 & 0.9422 & 0.9437 & 0.9500 & 0.9438 & 0.9450 & 0.9380 & 0.9421 & 0.9481 \\
\hline 1 & 0.99 & 2340 & 0.9968 & 0.9959 & 0.9959 & 0.9959 & 0.9961 & 0.9959 & 0.9960 & 0.9902 & 0.9959 & 0.9966 \\
\hline
\end{tabular}

Table S-11: MC Results versus Computation Results When CV=2

\begin{tabular}{|c|c|c|c|c|c|c|c|c|c|c|c|c|}
\hline $\mathrm{CV}$ & $\alpha$ & S & MC & Tradition & EXP & Hadley & Silver & J. et al. & $\begin{array}{l}\text { J. et al. } \\
\text { mod }\end{array}$ & $Z \& Z$ & Z\&Z app & $\begin{array}{l}\text { Trunc. } \\
\text { MC }\end{array}$ \\
\hline 2 & 0.3 & 686 & 0.2411 & negative & negative & 0.1973 & 0.3751 & 0.2239 & 0.2304 & 0.1613 & negative & 0.0855 \\
\hline 2 & 0.4 & 952 & 0.3539 & negative & negative & 0.3039 & 0.4806 & 0.3280 & 0.3592 & 0.2679 & negative & 0.1879 \\
\hline 2 & 0.5 & 1200 & 0.4608 & 0.0229 & 0.0240 & 0.4184 & 0.5803 & 0.4384 & 0.4805 & 0.3823 & 0.0229 & 0.3165 \\
\hline 2 & 0.6 & 1448 & 0.5754 & 0.3018 & 0.2948 & 0.5379 & 0.6751 & 0.5531 & 0.5927 & 0.5018 & 0.3018 & 0.4569 \\
\hline 2 & 0.7 & 1714 & 0.6964 & 0.5340 & 0.5279 & 0.6600 & 0.7654 & 0.6701 & 0.7040 & 0.6239 & 0.5340 & 0.6063 \\
\hline 2 & 0.8 & 2025 & 0.8117 & 0.7268 & 0.7244 & 0.7814 & 0.8506 & 0.7869 & 0.8119 & 0.7453 & 0.7268 & 0.7569 \\
\hline 2 & 0.9 & 2456 & 0.9135 & 0.88418349 & 0.8845 & 0.8982 & 0.9300 & 0.9001 & 0.9135 & 0.8621 & 0.8842 & 0.8952 \\
\hline 2 & 0.99 & 3479 & 0.9952 & 0.99170071 & 0.9917 & 0.9919 & 0.9940 & 0.9920 & 0.9932 & 0.9559 & 0.9917 & 0.9958 \\
\hline
\end{tabular}

Table S-12: MC Results versus Computation Results When CV=3

\begin{tabular}{|c|c|c|c|c|c|c|c|c|c|c|c|c|}
\hline $\mathrm{CV}$ & $\alpha$ & S & $\mathrm{MC}$ & Tradition & EXP & Hadley & Silver & J. et al. & $\begin{array}{l}\text { J. et al. } \\
\text { mod }\end{array}$ & $\mathrm{Z} \& \mathrm{Z}$ & $\mathrm{Z} \& \mathrm{Z}$ app & $\begin{array}{l}\text { Trunc. } \\
\text { MC }\end{array}$ \\
\hline
\end{tabular}




\begin{tabular}{|c|c|c|c|c|c|c|c|c|c|c|c|c|}
\hline 3 & 0.3 & 429 & 0.1621 & negative & negative & 0.0906 & 0.3492 & 0.1542 & 0.2226 & 0.0662 & negative & 0.0268 \\
\hline 3 & 0.4 & 828 & 0.2909 & negative & negative & 0.1847 & 0.4536 & 0.2450 & 0.3256 & 0.1603 & negative & 0.0967 \\
\hline 3 & 0.5 & 1200 & 0.4183 & negative & negative & 0.2970 & 0.5540 & 0.3493 & 0.4350 & 0.2725 & negative & 0.2078 \\
\hline 3 & 0.6 & 1572 & 0.5367 & negative & negative & 0.4238 & 0.6510 & 0.4653 & 0.5491 & 0.3994 & negative & 0.3500 \\
\hline 3 & 0.7 & 1971 & 0.6670 & 0.3009 & 0.2918 & 0.5625 & 0.7450 & 0.5917 & 0.6660 & 0.5380 & 0.3009 & 0.5141 \\
\hline 3 & 0.8 & 2437 & 0.7809 & 0.5900 & 0.5864 & 0.7089 & 0.8352 & 0.7259 & 0.7831 & 0.6844 & 0.5900 & 0.6870 \\
\hline 3 & 0.9 & 3083 & 0.9007 & 0.8260 & 0.8264 & 0.8589 & 0.9212 & 0.8652 & 0.8976 & 0.8345 & 0.8260 & 0.8546 \\
\hline 3 & 0.99 & 4619 & 0.9912 & 0.9876 & 0.9876 & 0.9882 & 0.9930 & 0.9883 & 0.9917 & 0.9638 & 0.9876 & 0.9863 \\
\hline
\end{tabular}

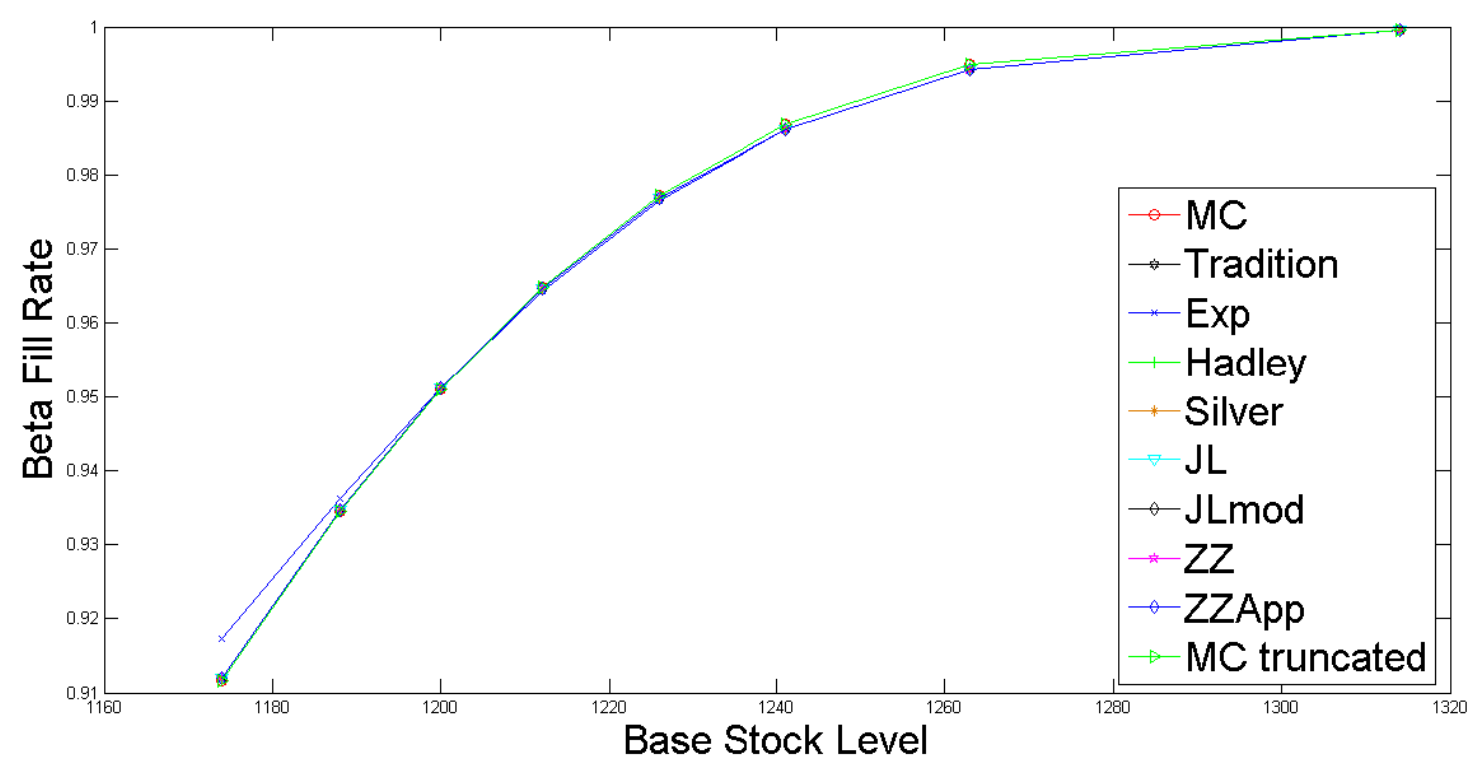

Figure S-1: V=0.1 Computation Results versus MC Results 


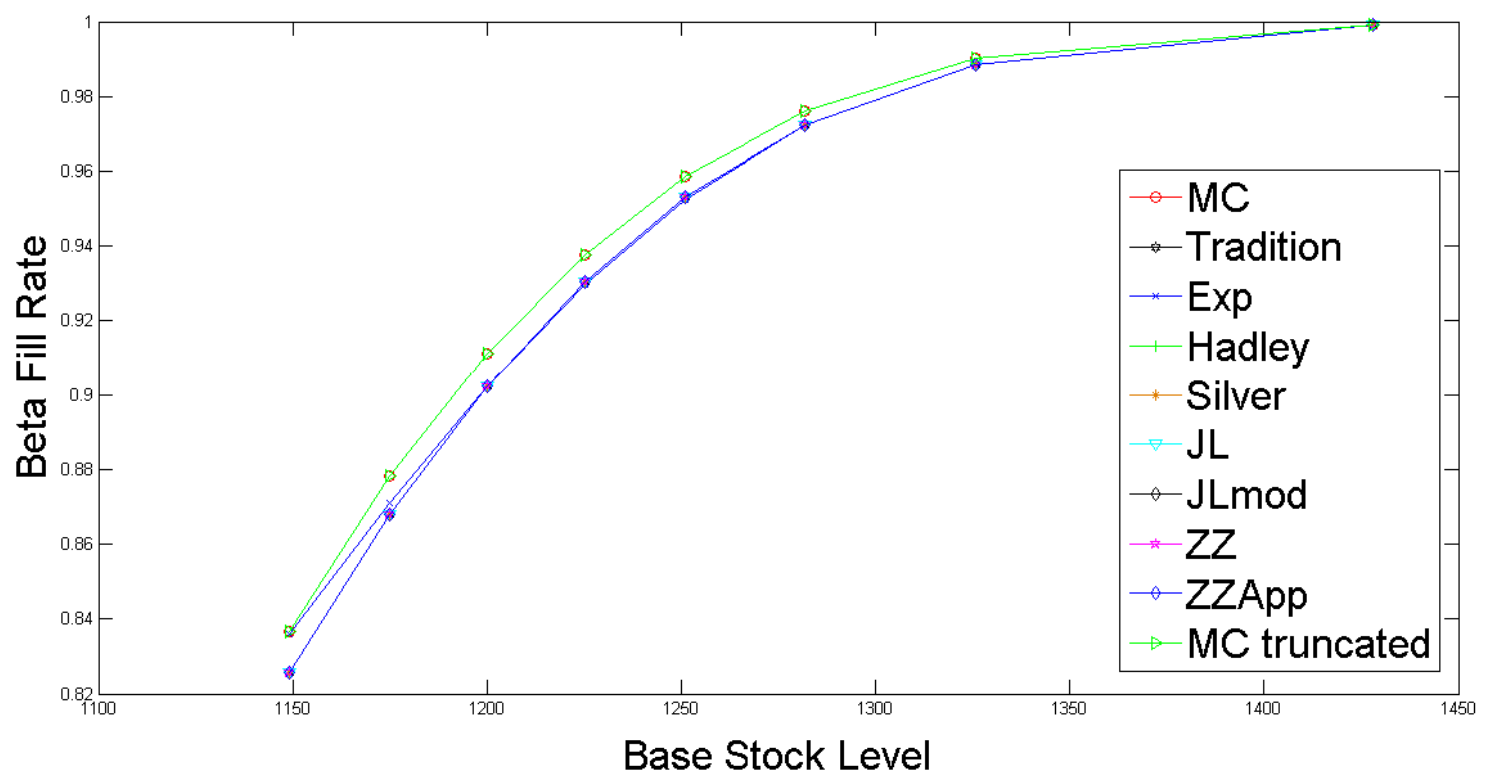

Figure S-2: CV=0.2 Computation Results versus MC Results

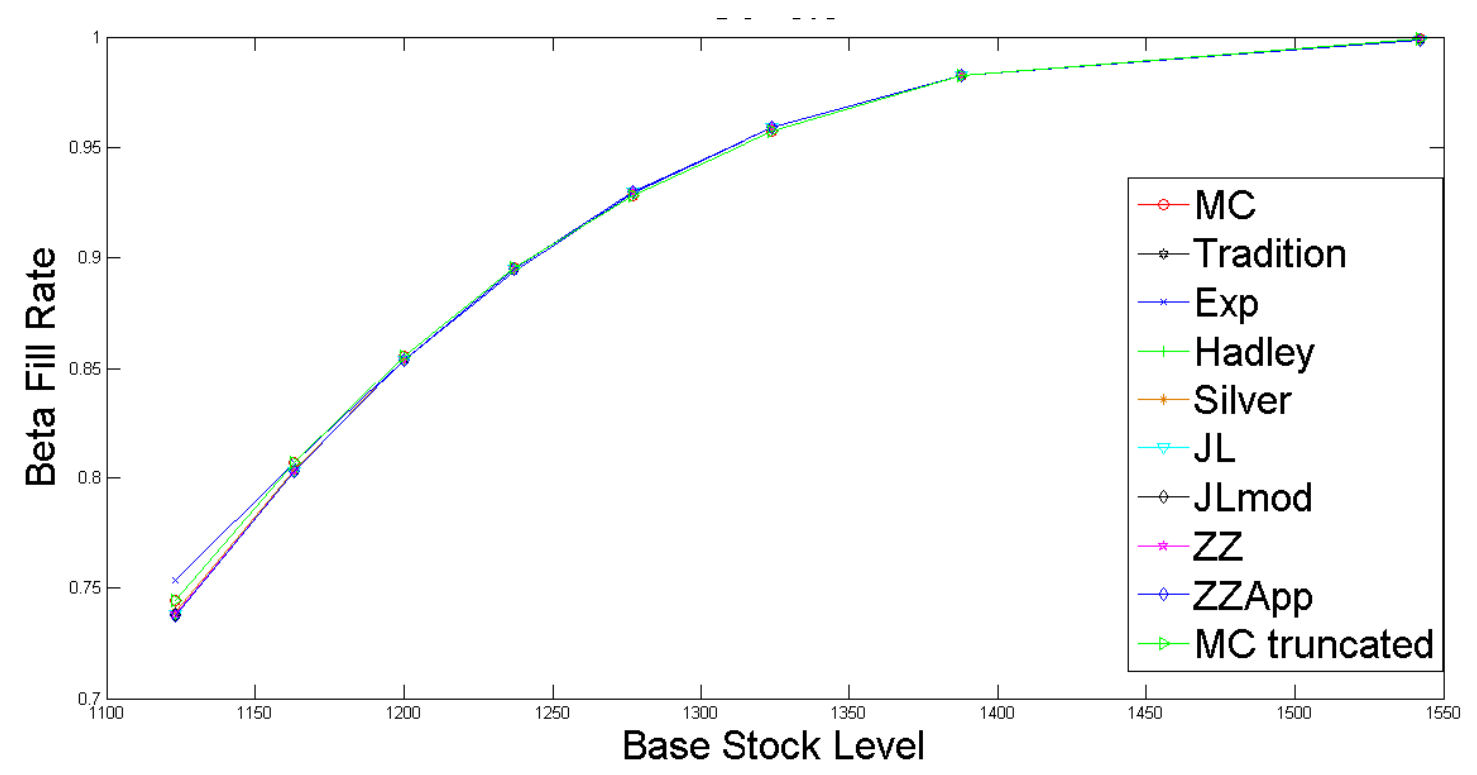

Figure S-3: CV=0.3 Computation Results versus MC Results 


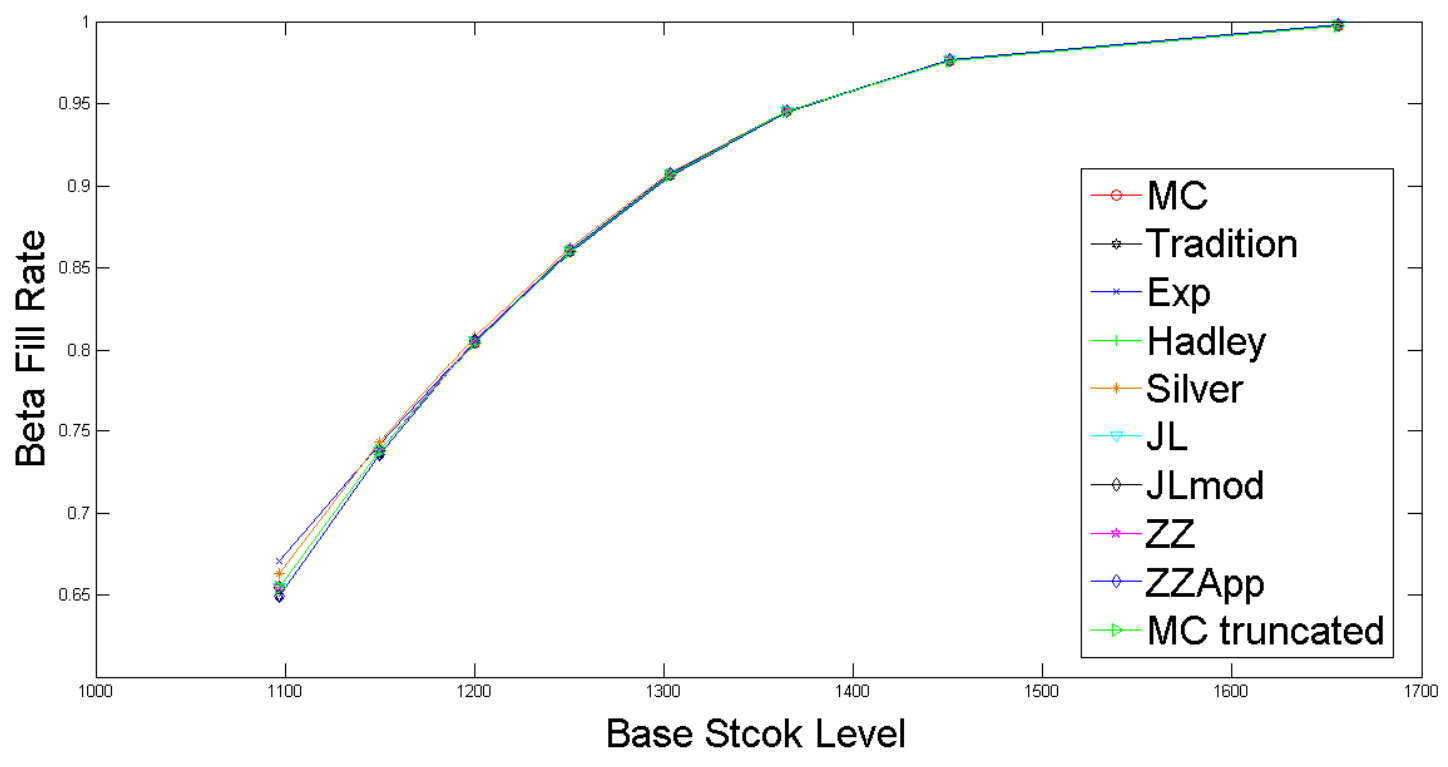

Figure S-4: CV=0.4 Computation Results versus MC Results

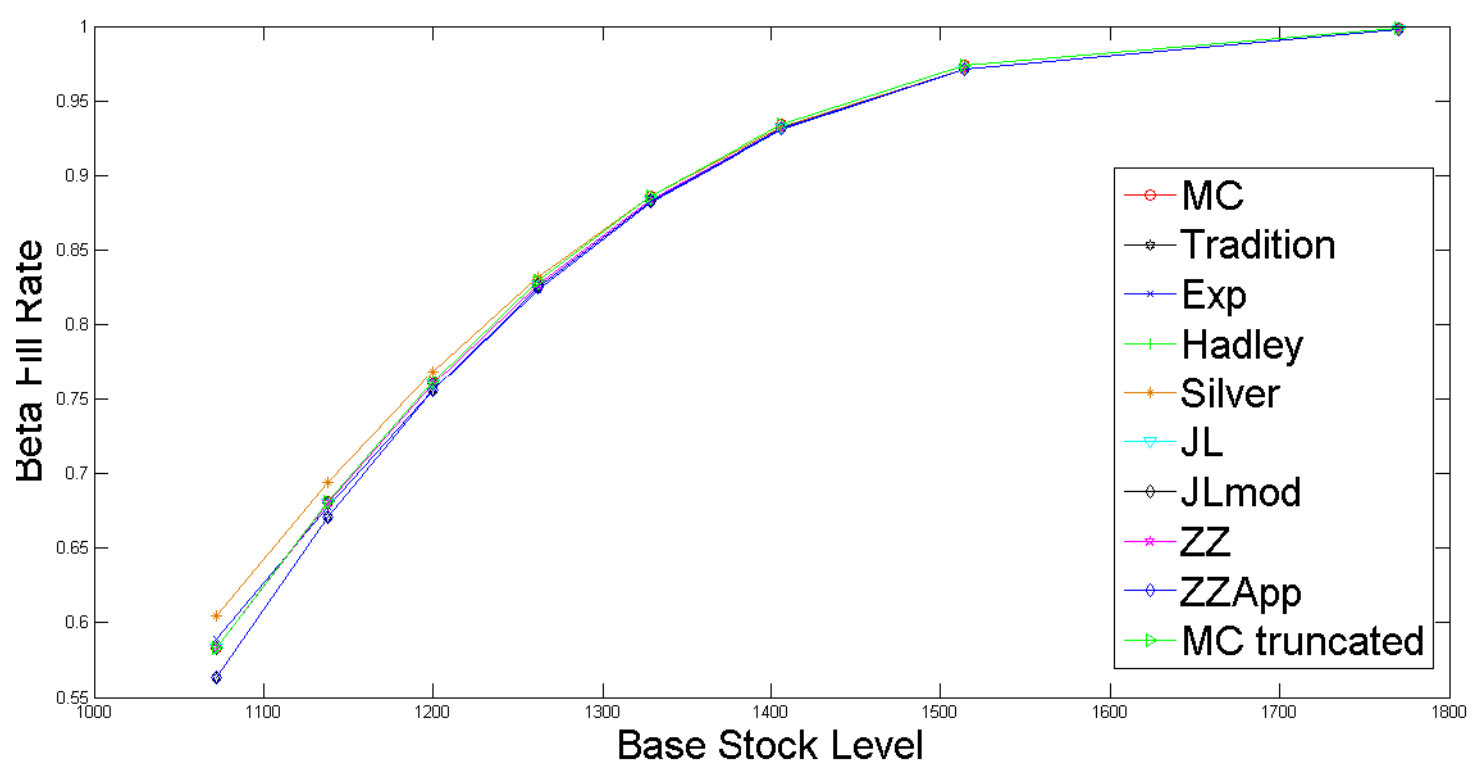

Figure S-5: CV=0.5 Computation Results versus MC Results 


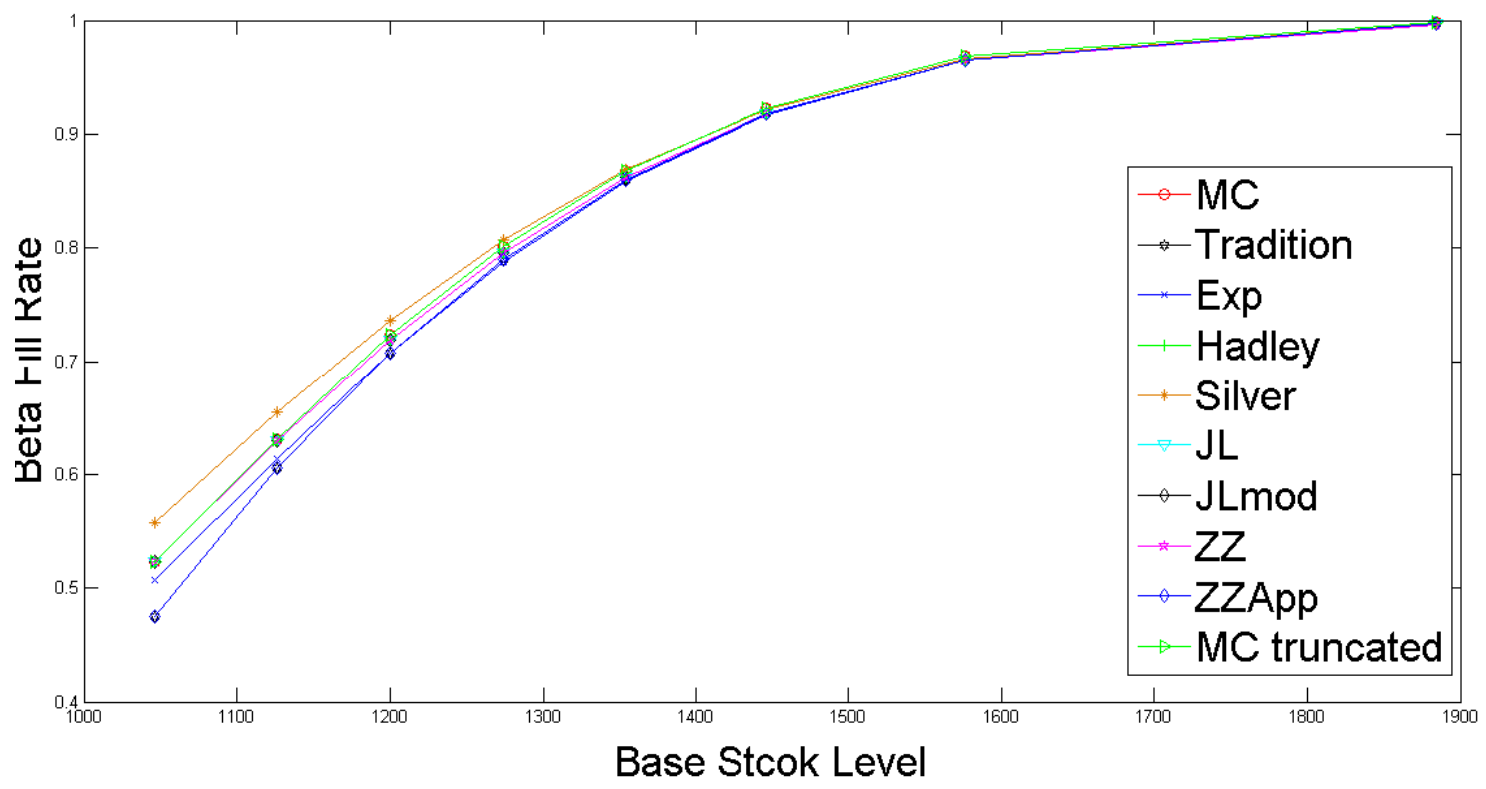

Figure S-6: CV=0.6 Computation Results versus MC Results

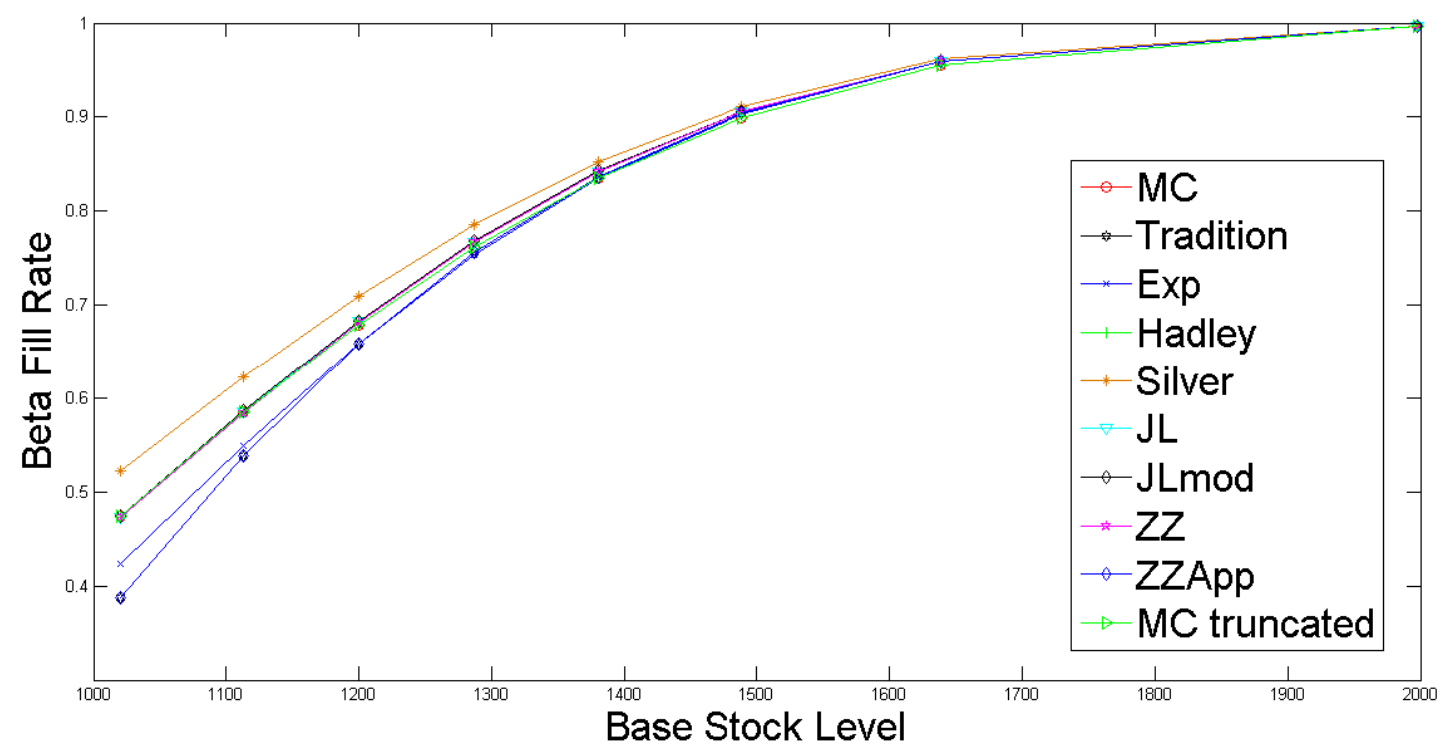

Figure S-7: CV=0.7 Computation Results versus MC Results 


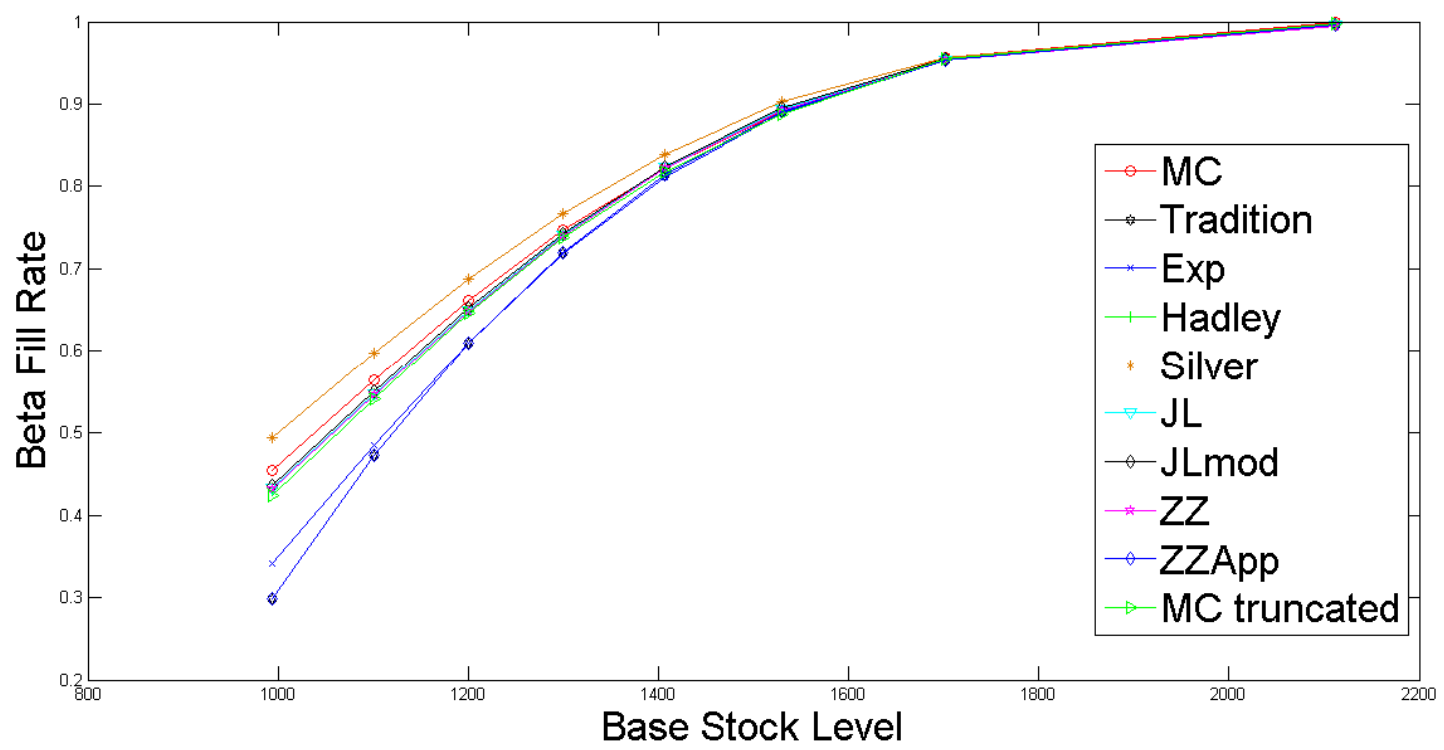

FigureS-8: CV=0.8 Computation Results versus MC Results

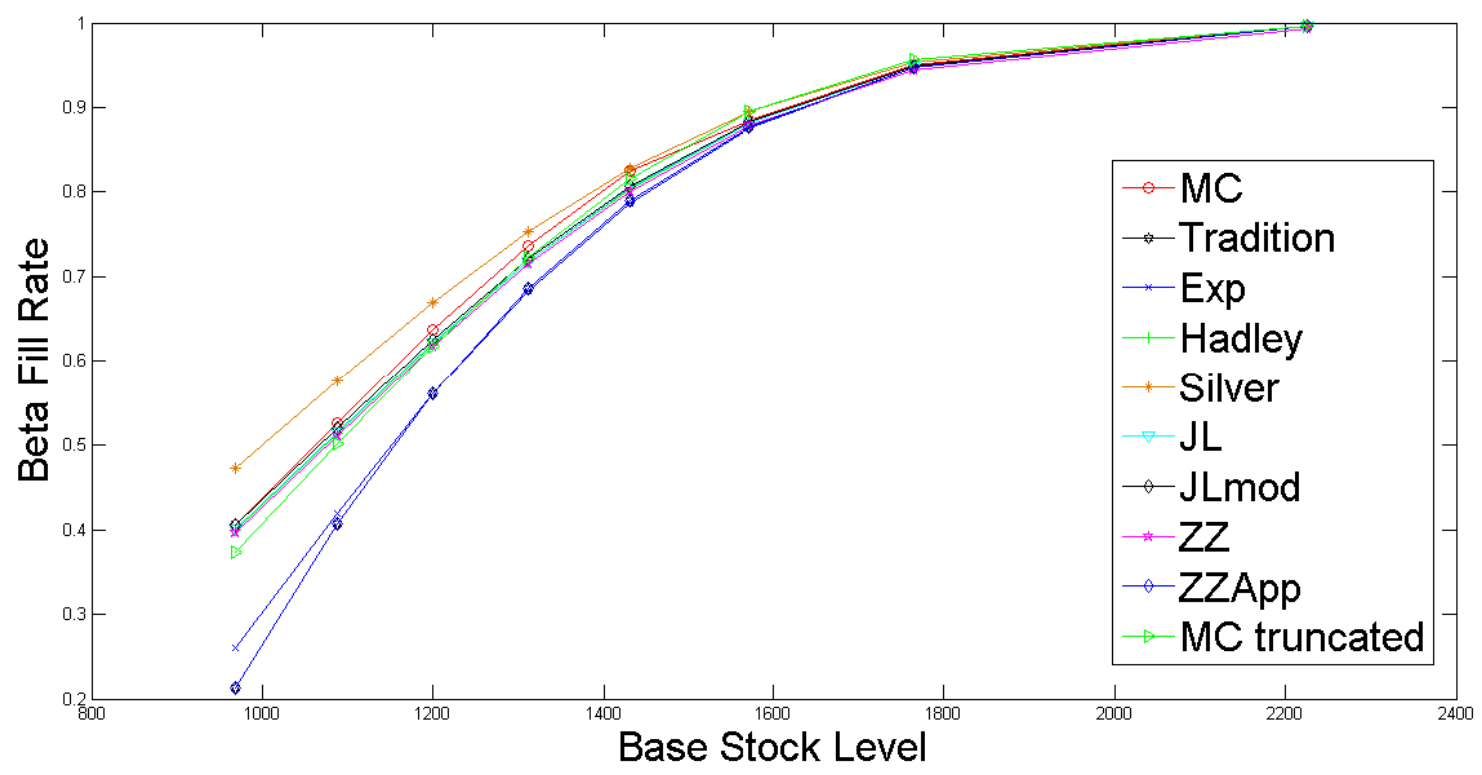

Figure S-9: CV=0.9 Computation Results versus MC Results 


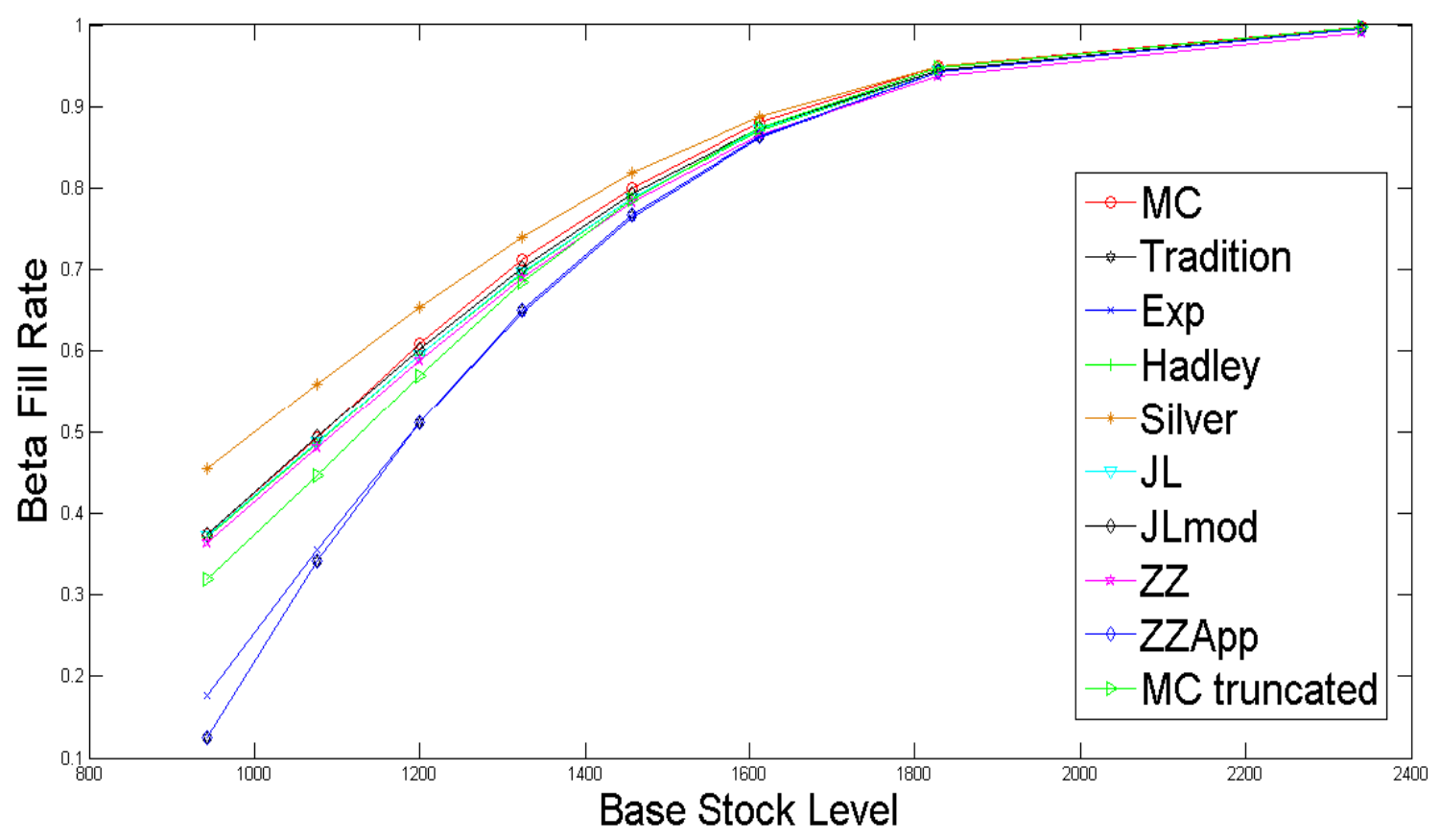

Figure S-10: CV=1 Computation Results versus MC Results

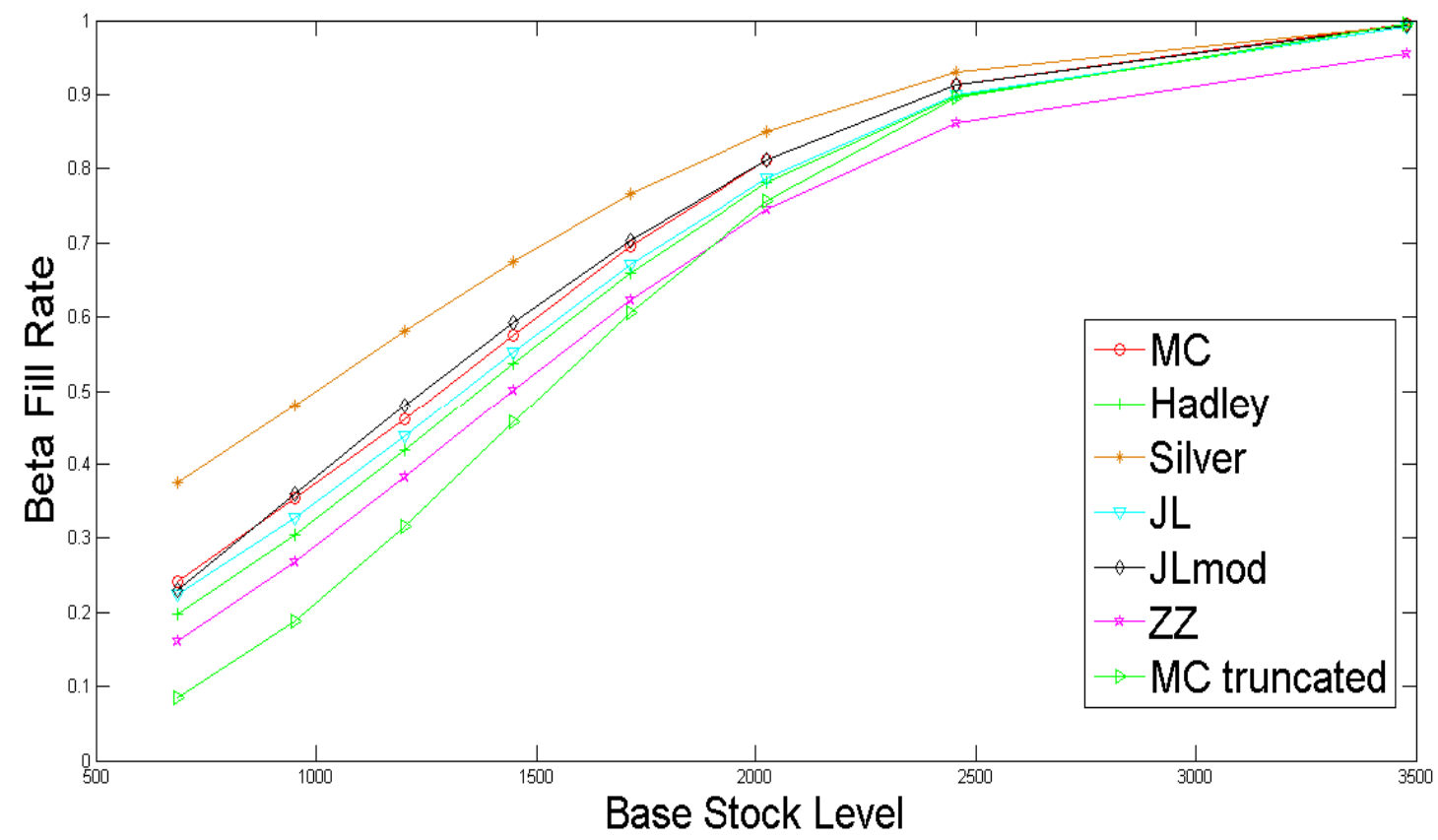

Figure S-11: CV=2 Computation Results versus MC Results 


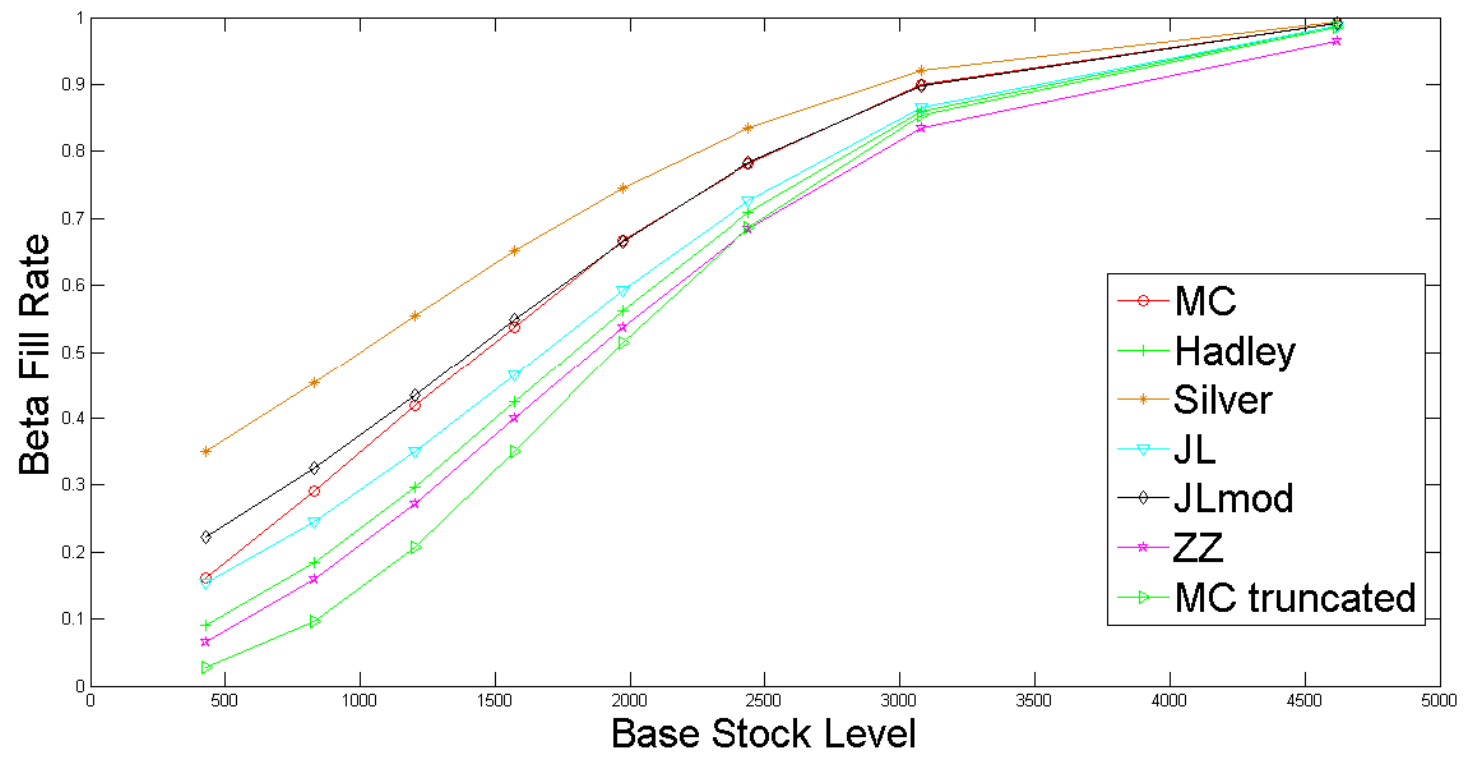

Figure S-12: CV=3 Computation Results versus MC Results 


\section{CURRICULUM VITAE}

Name:

Address:

Education:

Awards:
Peiyu Luo

Department of Industrial Engineering J.B. Speed School of Engineering

University of Louisville

Louisville, KY 40292

B.E., Civil Engineering

Jiangnan University, Wuxi, Jiangsu Province, CHN 2004-2008

University Fellowship Award 2011

University of Louisville, Louisville, KY 